\title{
Rh(III)-Catalyzed [4 + 2] Annulation of Indoles with Diazo Compounds: Access to Pyrimido[1,6-a]indole-1(2H)-Ones
}

\author{
Xun Chen, Xinwei Hu, Siyi Bai, Yuanfu Deng, Huanfeng Jiang, and Wei Zeng * \\ School of Chemistry and Chemical Engineering, South China University of Technology, Guangzhou \\ 510641, China \\ Correspondingauthor:zengwei@scut.edu.cn
}

\section{Supporting Information}

Table of Contents

1. General Experimental Information.

1.1. Table 1. Catalyst screening for the $[4+2]$ annulation of indole $(\mathbf{1 a})$ with diazo compound (2a)

1.2. Table 2. The effect of additives on the $[4+2]$ annulation of indole (1a) with diazo compound (2a)

1.3. Table 3. The effect of solvents on the $[4+2]$ annulation of indole $(\mathbf{1 a})$ with diazo compound (2a) .

1.4. Table 4. The effect of the reaction time on the $[4+2]$ annulation of indole (1a) with diazo compound $(\mathbf{2 a})$

1.5. Table 5. The effect of the reaction temperature on the $[4+2]$ annulation of indole $(\mathbf{1 a})$ with diazo compound (2a)

1.6. Table 6. The effect of proton sources on the $[4+2]$ annulation of indole (1a) with diazo compound (2a)

1.7. General procedure for the synthesis for pyrimido[1,6-a]indole-1(2H)-ones derivatives $(\mathbf{3 a - 3 z})$

2. Synthetic Application for This Transformation.........................................................................16

3. Controlled Experiments for Mechanism Studies. ............................................................... 19

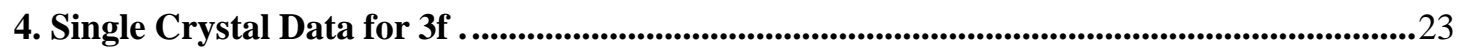

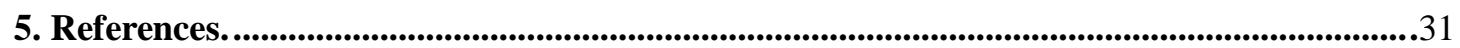

6. ${ }^{1} \mathrm{H}$ NMR and ${ }^{13} \mathrm{C}$ NMR Spectrum for All Isolated Products. ......................................................332 


\section{General experimental information}

All reactions were carried out in flame-dried sealed tubes with magnetic stirring. Unless otherwise noted, all experiments were performed under argon atmosphere. All reagents were purchased from TCI, Acros or Strem. Solvents were treated with $4 \AA$ molecular sieves or sodium and distilled prior to use. The starting materials ( $N$-carbamoyl indoles substrates) $\mathbf{1 a - 1 q}, \mathbf{1 t}, \mathbf{1 z}$ and diazo compound $\mathbf{2} \mathbf{r}-\mathbf{2 y}$ were prepared according to the previously reported procedures. ${ }^{[1,2]}$ Purifications of reaction products were carried out by flash chromatography using Qingdao Haiyang Chemical Co. Ltd silica gel (40-63 mm). Infrared spectra (IR) were recorded on a Brucker TENSOR 27 FTIR spectrophotometer and are reported as wavelength numbers $\left(\mathrm{cm}^{-1}\right)$. Infrared spectra were recorded by preparing a $\mathrm{KBr}$ pellet containing the title compound. ${ }^{1} \mathrm{H}$ NMR and ${ }^{13} \mathrm{C}$ NMR spectra were recorded with tetramethylsilane (TMS) as internal standard at ambient temperature unless otherwise indicated on a Bruker Avance DPX 600 fourier Transform spectrometer operating at 400 $\mathrm{MHz}$ for ${ }^{1} \mathrm{H}$ NMR and $100 \mathrm{MHz}$ for ${ }^{13} \mathrm{C}$ NMR. Chemical shifts are reported in parts per million (ppm) and coupling constants are reported as Hertz (Hz). Splitting patterns are designated as singlet (s), broad singlet (bs), doublet (d), triplet (t). Splitting patterns that could not be interpreted or easily visualized are designated as multiple $(\mathrm{m})$. Low resolution mass spectra were recorded using a Waters HPLC/ZQ4000 Mass Spectrometer. High resolution mass spectra (HRMS) were recorded on an IF-TOF spectrometer (Micromass). Gas chromatograph mass spectra were obtained with a SHIMADZU model GCMS-QP5000 spectrometer. Crystal data were collected on a Bruker D8 Advance employing graphite monochromated Mo - K $\alpha$ radiation $(\lambda=0.71073 \AA)$ at 293 (2) $\mathrm{K}$ and operating in the $\varphi$ - $\omega$ scan mode. The structure was solved by direct methods SHELXS-97. 
1.1. Table 1. Catalyst screening for the $[4+2]$ annulation of indole (1a) with diazo compound (2a) ${ }^{a}$

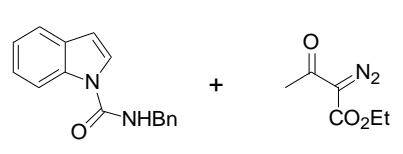

$1 a$

$2 a$

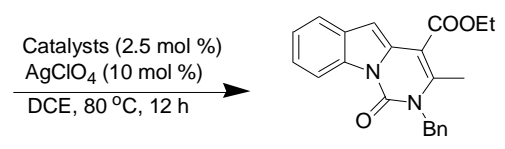

$3 a$

\begin{tabular}{ccc}
\hline entry & catalyst & yield $(\%)^{b}$ \\
\hline 1 & {$\left[\mathrm{Cp}^{*} \mathrm{IrCl}_{2}\right]_{2}$} & 17 \\
2 & $\mathrm{Cp}^{*} \mathrm{Co}(\mathrm{CO}) \mathrm{I}_{2}$ & 0 \\
3 & {$\left[\left\{\mathrm{RuCl}_{2}(\mathrm{p}-\mathrm{cymene})\right\}_{2}\right]$} & 0 \\
4 & $\mathrm{Pd}(\mathrm{OAc})_{2}$ & 0 \\
5 & $\mathrm{RhCl}_{3}$ & 0 \\
6 & $\mathrm{Rh}_{2}(\mathrm{COD})_{2} \mathrm{Cl}_{2}$ & 0 \\
7 & {$\left[\mathrm{Cp} * \mathrm{RhCl}_{2}\right]_{2}$} & 52 \\
\hline
\end{tabular}

$\overline{{ }^{a}}$ All the reactions were carried out using $N$-carbamoyl indole 1a $(0.1 \mathrm{mmol})$, diazo compound 2a $(0.2 \mathrm{mmol})$, catalyst $(2.5 \mathrm{~mol} \%), \mathrm{AgClO}_{4}(10 \mathrm{~mol} \%)$ in 1, 2-dichloroethane (DCE) (2.0 mL) at $80{ }^{\circ} \mathrm{C}$ for $12 \mathrm{~h}$ in a sealed reaction tube, followed by flash chromatography on $\mathrm{SiO}_{2} \cdot{ }^{b}$ Isolated yield.

1.2. Table 2. The effect of additives on the $[4+2]$ annulation of indole (1a) with diazo compound (2a) ${ }^{a}$

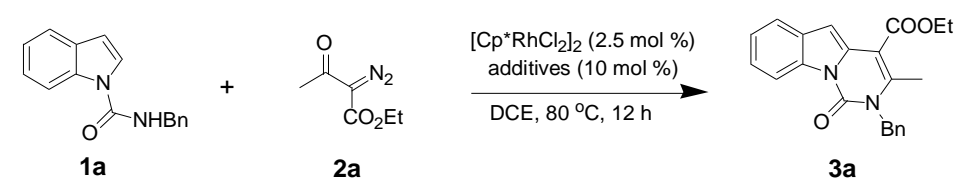

\begin{tabular}{ccc}
\hline entry & additives & yield (\%) \\
\hline 1 & $\mathrm{AgClO}_{4}$ & 52 \\
2 & $\mathrm{AgBF}_{4}$ & 55 \\
3 & $\mathrm{AgNTf}_{2}$ & 62 \\
4 & $\mathrm{AgOAc}$ & 58 \\
5 & $\mathrm{AgSbF}_{6}$ & 64 \\
\hline
\end{tabular}

${ }^{a}$ All the reactions were carried out using $N$-carbamoyl indole 1a $(0.1 \mathrm{mmol})$, diazo compound 2a $(0.2 \mathrm{mmol}),\left[\mathrm{Cp} * \mathrm{RhCl}_{2}\right]_{2}(2.5 \mathrm{~mol} \%)$, additives $(10 \mathrm{~mol} \%)$ in DCE $(2.0 \mathrm{~mL})$ at $80{ }^{\circ} \mathrm{C}$ for $12 \mathrm{~h}$ in a sealed reaction tube, followed by flash chromatography on $\mathrm{SiO}_{2} \cdot{ }^{b}$ Isolated yield.

1.3. Table 3. The effect of solvents on the $[4+2]$ annulation of indole (1a) with diazo compound (2a) ${ }^{a}$

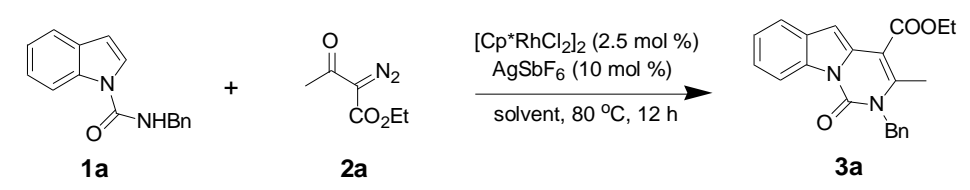

\begin{tabular}{ccc}
\hline entry & solvent & yield $(\%)^{b}$ \\
\hline 1 & DCE & 64 \\
2 & EtOH & 22 \\
3 & MeCN & 17 \\
4 & toluene & trace \\
5 & THF & trace \\
\hline
\end{tabular}

\footnotetext{
${ }^{a}$ All the reactions were carried out using $N$-carbamoyl indole 1a $(0.1 \mathrm{mmol})$, diazo compound 2a (0.2 mmol), $\left[\mathrm{Cp}^{*} \mathrm{RhCl}_{2}\right]_{2}(1.6 \mathrm{mg}, 2.5 \mathrm{~mol} \%), \operatorname{AgSbF}_{6}(10 \mathrm{~mol} \%)$ in solvent $(2.0 \mathrm{~mL})$ at $80{ }^{\circ} \mathrm{C}$ for $12 \mathrm{~h}$ in a sealed reaction tube, followed by flash chromatography on $\mathrm{SiO}_{2} \cdot{ }^{b}$ Isolated yield.
} 
1.4. Table 4. The effect of the reaction time on the $[4+2]$ annulation of indole (1a) with diazo compound (2a) ${ }^{a}$
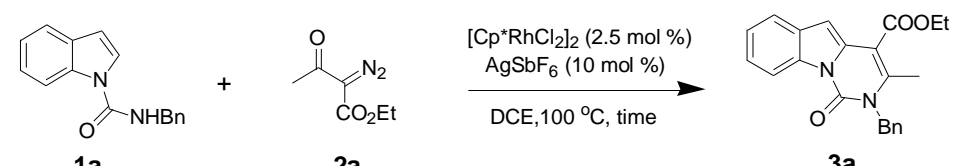

$2 a$

3a

\begin{tabular}{ccc}
\hline entry & time $(\mathrm{h})$ & yield $(\%)^{b}$ \\
\hline 1 & 12 & 64 \\
2 & 24 & 66 \\
3 & 36 & 63 \\
\hline
\end{tabular}

${ }^{a}$ All the reactions were carried out using $N$-carbamoyl indole 1a $(0.1 \mathrm{mmol})$, diazo compound 2a (0.2 mmol), $\left[\mathrm{Cp}^{*} \mathrm{RhCl}_{2}\right]_{2}(2.5 \mathrm{~mol} \%), \mathrm{AgSbF}_{6}(10 \mathrm{~mol} \%)$ in DCE $(2.0 \mathrm{~mL})$ at $80{ }^{\circ} \mathrm{C}$ for the given time in a sealed reaction tube, followed by flash chromatography on $\mathrm{SiO}_{2} \cdot{ }^{b}$ Isolated yield.

1.5. Table 5. The effect of the reaction temperature on the $[4+2]$ annulation of indole (1a) with diazo compound (2a) ${ }^{a}$

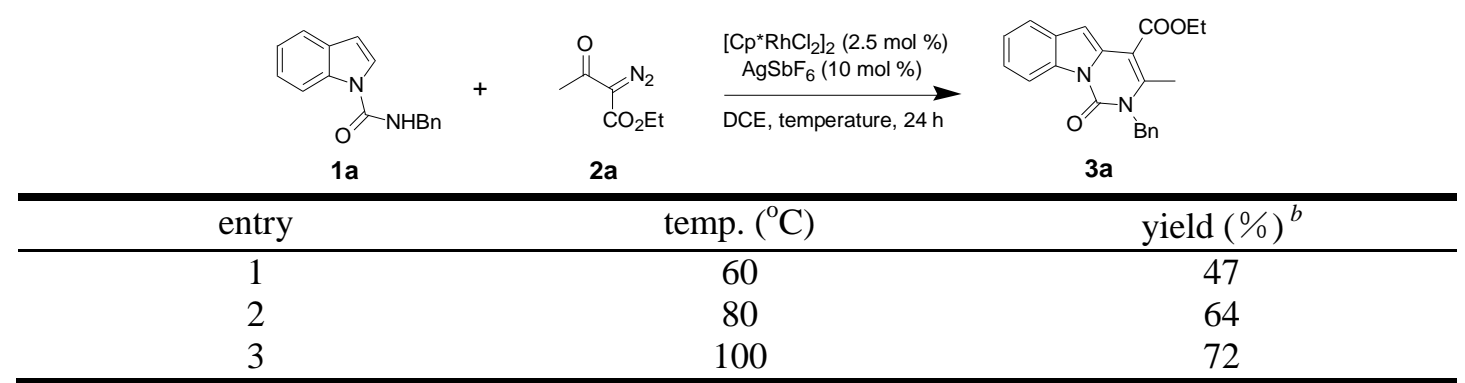

${ }^{a}$ All the reactions were carried out using $N$-carbamoyl indole 1a $(0.1 \mathrm{mmol})$, diazo compound 2a $(0.2 \mathrm{mmol}),\left[\mathrm{Cp} * \mathrm{RhCl}_{2}\right]_{2}(2.5 \mathrm{~mol} \%), \mathrm{AgSbF}_{6}(10 \mathrm{~mol} \%)$ in DCE $(2.0 \mathrm{~mL})$ at the given temperature for $24 \mathrm{~h}$ in a sealed reaction tube, followed by flash chromatography on $\mathrm{SiO}_{2} .{ }^{b}$ Isolated yield.

1.6. Table 6. The effect of proton sources on the $[4+2]$ annulation of indole (1a) with diazo compound (2a) ${ }^{a}$

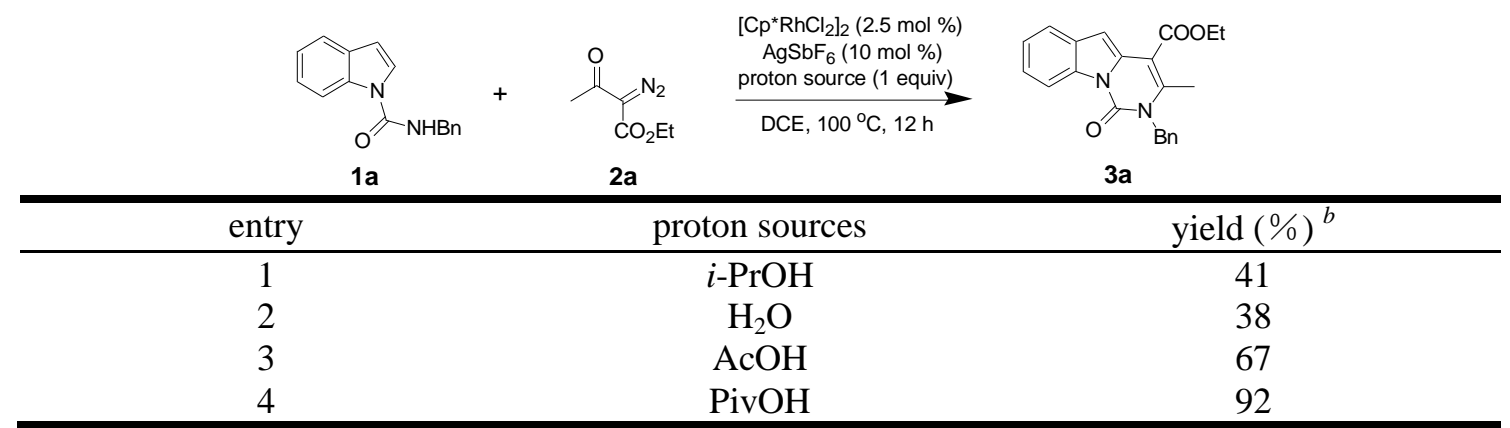

\footnotetext{
${ }^{a}$ All the reactions were carried out using $N$-carbamoyl indole 1a $(0.1 \mathrm{mmol})$, diazo compound 2a (0.2 mmol), [Cp* $\left.\mathrm{RhCl}_{2}\right]_{2}(1.6 \mathrm{mg}, 2.5 \mathrm{~mol} \%), \mathrm{AgSbF}_{6}(10 \mathrm{~mol} \%)$, proton sources (1 equiv) in solvent $(2.0 \mathrm{~mL})$ at $100{ }^{\circ} \mathrm{C}$ for $24 \mathrm{~h}$ in a sealed reaction tube, followed by flash chromatography on $\mathrm{SiO}_{2} .{ }^{b}$ Isolated yield.
}

1.7. General procedure for the synthesis for pyrimido[1,6-a]indole-1(2H)-ones derivatives (3a-3z) 


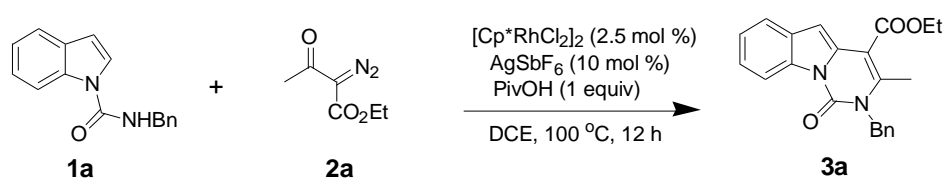

A $10 \mathrm{~mL}$ of reaction tube was charged with $N$-carbamoyl indoles $1(0.1 \mathrm{mmol}),\left[\mathrm{Cp} * \mathrm{RhCl}_{2}\right]_{2}(1.6$ $\mathrm{mg}, 2.5 \mathrm{~mol} \%), \operatorname{AgSbF}_{6}(3.4 \mathrm{mg}, 10 \mathrm{~mol} \%)$, PivOH (10.2 mg, 1 equiv) and DCE (1.5 mL) under Ar atmosphere. Then diazo compound $2(0.2 \mathrm{mmol})$ in DCE $(0.5 \mathrm{~mL})$ was added in one-pot under $\mathrm{Ar}$ and the mixture was stirred at $100{ }^{\circ} \mathrm{C}$ for $24 \mathrm{~h}$. The corresponding reaction mixture was filtered through a pad of Celite, washed with EtOAc and concentrated under reduced pressure. The residue was purified by flash chromatography on silical gel using ethyl acetate/petroleum ether as eluent to afford the desired products 3 .

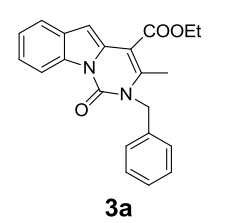

Ethyl 2-benzyl-3-methyl-1-oxo-1,2-dihydropyrimido[1,6-a]indole-4-carboxylate (3a) : White solid; $33.1 \mathrm{mg}$, 92\% yield; m.p. 144.1-144.9 ${ }^{\circ} \mathrm{C} .{ }^{1} \mathrm{H}$ NMR $\left(400 \mathrm{MHz}, \mathrm{CDCl}_{3}\right) \delta 8.65(\mathrm{~d}, J=7.8 \mathrm{~Hz}$, 1H), 7.65 (d, $J=7.4 \mathrm{~Hz}, 1 \mathrm{H}), 7.34$ (dt, $J=13.4,7.0 \mathrm{~Hz}, 4 \mathrm{H}), 7.24$ (d, $J=6.4 \mathrm{~Hz}, 1 \mathrm{H}), 7.19$ (d, $J=$ $7.4 \mathrm{~Hz}, 2 \mathrm{H}), 6.86(\mathrm{~s}, 1 \mathrm{H}), 5.35$ (s, 2H), 4.42 (q, J=7.0 Hz, 2H), $2.51(\mathrm{~s}, 3 \mathrm{H}), 1.44$ (t, $J=7.0 \mathrm{~Hz}$, $3 \mathrm{H}) ;{ }^{13} \mathrm{C}$ NMR $\left(101 \mathrm{MHz}, \mathrm{CDCl}_{3}\right) \delta 165.8,148.3,143.0,136.5,133.0,131.6,130.6,129.0,127.6$, $126.0,124.2,122.7,119.9,116.3,104.5,99.9,61.3,47.5,17.3,14.3$; HR-MS (ESI) calcd for [M + $1]^{+}: \mathrm{C}_{22} \mathrm{H}_{21} \mathrm{~N}_{2} \mathrm{O}_{3}: 361.1547$, found: 361.1546; IR (KBr): 2926, 1695, 1616, 1495, 1452, 1417 , $1372,1316,1235,1055 \mathrm{~cm}^{-1}$.

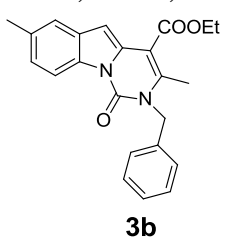

Ethyl 2-benzyl-3,7-dimethyl-1-oxo-1,2-dihydropyrimido[1,6-a]indole-4-carboxylate $(3 \mathrm{~b})$ : White solid; $33.6 \mathrm{mg}, 90 \%$ yield; m.p. $152.3-152.7{ }^{\circ} \mathrm{C} .{ }^{1} \mathrm{H} \mathrm{NMR}\left(400 \mathrm{MHz}, \mathrm{CDCl}_{3}\right) \delta 8.50(\mathrm{~d}, J=$ $8.4 \mathrm{~Hz}, 1 \mathrm{H}), 7.43(\mathrm{~s}, 1 \mathrm{H}), 7.34-7.29(\mathrm{~m}, 2 \mathrm{H}), 7.25(\mathrm{~d}, J=3.3 \mathrm{~Hz}, 1 \mathrm{H}), 7.17(\mathrm{dd}, J=16.5,7.9 \mathrm{~Hz}$, $3 \mathrm{H}), 6.78(\mathrm{~s}, 1 \mathrm{H}), 5.36(\mathrm{~s}, 2 \mathrm{H}), 4.42(\mathrm{q}, J=7.1 \mathrm{~Hz}, 2 \mathrm{H}), 2.52(\mathrm{~s}, 3 \mathrm{H}), 2.49(\mathrm{~s}, 3 \mathrm{H}), 1.44(\mathrm{t}, J=7.1$ $\mathrm{Hz}, 3 \mathrm{H}) ;{ }^{13} \mathrm{C}$ NMR $\left(101 \mathrm{MHz}, \mathrm{CDCl}_{3}\right) \delta 165.9,148.2,142.7,136.6,133.8,131.7,131.3,130.9$, 129.0, 127.5, 126.0, 124.3, 119.6, 115.8, 104.6, 99.6, 61.3, 47.5, 21.6, 17.3, 14.3; HR-MS (ESI) calcd for $[\mathrm{M}+1]^{+}: \mathrm{C}_{23} \mathrm{H}_{23} \mathrm{~N}_{2} \mathrm{O}_{3}: 375.1703$, found: 375.1702; IR (KBr): 2923, 1692, 1600, 1416, $1358,1294,1230,1187,1107,1053 \mathrm{~cm}^{-1}$. 


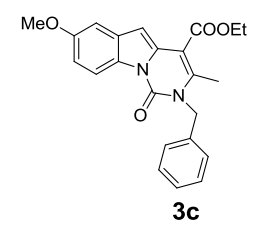

Ethyl 2-benzyl-7-methoxy-3-methyl-1-oxo-1,2-dihydropyrimido[1,6-a]indole-4-carboxylate (3c) : White solid; $36.6 \mathrm{mg}, 94 \%$ yield; m.p. 164.1-165.0 ${ }^{\circ} \mathrm{C} .{ }^{1} \mathrm{H} \mathrm{NMR}\left(400 \mathrm{MHz}, \mathrm{CDCl}_{3}\right) \delta 8.52$ $(\mathrm{d}, J=9.0 \mathrm{~Hz}, 1 \mathrm{H}), 7.32(\mathrm{t}, J=7.3 \mathrm{~Hz}, 2 \mathrm{H}), 7.26(\mathrm{~d}, J=7.6 \mathrm{~Hz}, 1 \mathrm{H}), 7.20(\mathrm{~d}, J=7.5 \mathrm{~Hz}, 2 \mathrm{H})$, 7.09 (s, 1H), 6.95 (d, $J=9.0 \mathrm{~Hz}, 1 \mathrm{H}), 6.80(\mathrm{~s}, 1 \mathrm{H}), 5.38$ (s, 2H), 4.43 (q, $J=7.1 \mathrm{~Hz}, 2 \mathrm{H}), 3.88$ (s, $3 \mathrm{H}), 2.53(\mathrm{~s}, 3 \mathrm{H}), 1.45(\mathrm{t}, J=7.1 \mathrm{~Hz}, 3 \mathrm{H}) ;{ }^{13} \mathrm{C} \mathrm{NMR}\left(101 \mathrm{MHz}, \mathrm{CDCl}_{3}\right) \delta 165.8,157.0,148.0$, 142.9, 136.5, 132.3, 131.7, 129.0, 127.8, 127.6, 126.0, 117.0, 112.0, 104.4, 101.8, 99.7, 61.3, 55.6, 47.6, 17.3, 14.3; HR-MS (ESI) calcd for [M + 1] ${ }^{+}: \mathrm{C}_{23} \mathrm{H}_{23} \mathrm{~N}_{2} \mathrm{O}_{4}: 391.1652$, found: 391.1654; IR (KBr): 2926, 1692, 1600, 1478, 1418, 1417, 1378, 1309, 1237, $1032 \mathrm{~cm}^{-1}$.

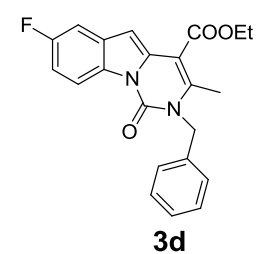

Ethyl 2-benzyl-7-fluoro-3-methyl-1-oxo-1,2-dihydropyrimido[1,6-a]indole-4-carboxylate (3d) : Reddish solid; $18.1 \mathrm{mg}, 48 \%$ yield; m.p. 169.1-169.5 ${ }^{\circ} \mathrm{C} .{ }^{1} \mathrm{H}$ NMR $\left(400 \mathrm{MHz}, \mathrm{CDCl}_{3}\right) \delta$ 8.59 (dd, $J=8.8,4.7 \mathrm{~Hz}, 1 \mathrm{H}), 7.34(\mathrm{t}, J=7.3 \mathrm{~Hz}, 2 \mathrm{H}), 7.28(\mathrm{~d}, J=7.6 \mathrm{~Hz}, 2 \mathrm{H}), 7.20$ (d, $J=7.4$ Hz, 2H), 7.05 (t, $J=9.1 \mathrm{~Hz}, 1 \mathrm{H}), 6.83(\mathrm{~s}, 1 \mathrm{H}), 5.40(\mathrm{~s}, 2 \mathrm{H}), 4.44(\mathrm{q}, J=7.0 \mathrm{~Hz}, 2 \mathrm{H}), 2.56(\mathrm{~s}, 3 \mathrm{H})$, $1.45(\mathrm{t}, J=7.1 \mathrm{~Hz}, 3 \mathrm{H}) ;{ }^{13} \mathrm{C}$ NMR $\left(101 \mathrm{MHz}, \mathrm{CDCl}_{3}\right) \delta 165.6,160.2(\mathrm{~d}, J=240.4 \mathrm{~Hz}), 148.1$, 143.5, 136.3, 133.1, 131.7, 131.6 (d, $J=11.1 \mathrm{~Hz}), 129.4,129.0,127.7,126.0,117.4(\mathrm{~d}, J=9.1$ Hz), $110.8(\mathrm{~d}, J=25.3 \mathrm{~Hz}), 104.9(\mathrm{~d}, J=23.2 \mathrm{~Hz}), 104.4,99.6,99.6(\mathrm{~d}, J=4.0 \mathrm{~Hz}), 61.4,47.7$, 17.3, 14.3; HR-MS (ESI) calcd for [M + 1] ${ }^{+}: \mathrm{C}_{22} \mathrm{H}_{20} \mathrm{FN}_{2} \mathrm{O}_{3}: 379.1452$, found: 379.1449 ; IR (KBr): 2923, 1711, 1689, 1591, 1446, 1416, 1376, 1359, 1228, $1051 \mathrm{~cm}^{-1}$.

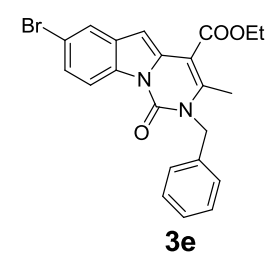

Ethyl 2-benzyl-7-bromo-3-methyl-1-oxo-1,2-dihydropyrimido[1,6-a]indole-4-carboxylate (3e) : Reddish solid; $23.6 \mathrm{mg}, 54 \%$ yield; m.p. 183.2-187.7 ${ }^{\circ} \mathrm{C} .{ }^{1} \mathrm{H}$ NMR $\left(400 \mathrm{MHz}, \mathrm{CDCl}_{3}\right) \delta$ $8.50(\mathrm{~d}, J=8.8 \mathrm{~Hz}, 1 \mathrm{H}), 7.78(\mathrm{~s}, 1 \mathrm{H}), 7.41(\mathrm{~d}, J=8.8 \mathrm{~Hz}, 1 \mathrm{H}), 7.34(\mathrm{t}, J=7.2 \mathrm{~Hz}, 2 \mathrm{H}), 7.29(\mathrm{~d}, J$ $=7.0 \mathrm{~Hz}, 2 \mathrm{H}), 7.21(\mathrm{~d}, J=7.3 \mathrm{~Hz}, 2 \mathrm{H}), 6.80(\mathrm{~s}, 1 \mathrm{H}), 5.39(\mathrm{~s}, 2 \mathrm{H}), 4.44(\mathrm{q}, J=6.9 \mathrm{~Hz}, 2 \mathrm{H}), 2.57(\mathrm{~s}$ $3 \mathrm{H}), 1.45(\mathrm{t}, J=7.1 \mathrm{~Hz}, 3 \mathrm{H}) ;{ }^{13} \mathrm{C}$ NMR $\left(101 \mathrm{MHz}, \mathrm{CDCl}_{3}\right) \delta 165.5,148.0,143.7,136.2,132.7$, 132.3, 131.6, 129.0, 127.7, 126.0, 125.6, 122.3, 117.7, 117.6, 104.4, 99.0, 61.4, 47.7, 17.4, 14.3; 
HR-MS (ESI) calcd for $[\mathrm{M}+1]^{+}: \mathrm{C}_{22} \mathrm{H}_{20} \mathrm{BrN}_{2} \mathrm{O}_{3}: 439.0652$, found: 439.0656; IR (KBr): 2925, $1695,1588,1444,1415,1357,1291,1233,1053 \mathrm{~cm}^{-1}$.

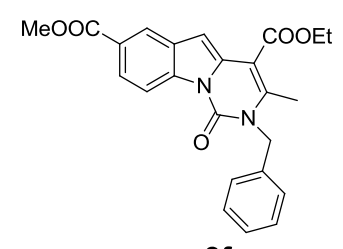

$3 \mathbf{f}$

4-Ethyl 7-methyl 2-benzyl-3-methyl-1-oxo-1,2-dihydropyrimido[1,6-a]indole-4,7dicarboxylate (3f) : White solid; $30.2 \mathrm{mg}, 72 \%$ yield; m.p. $156.3-156.8{ }^{\circ} \mathrm{C} .{ }^{1} \mathrm{H}$ NMR (400 MHz, $\left.\mathrm{CDCl}_{3}\right) \delta 8.64(\mathrm{~d}, J=8.7 \mathrm{~Hz}, 1 \mathrm{H}), 8.36(\mathrm{~s}, 1 \mathrm{H}), 7.99(\mathrm{~d}, J=8.7 \mathrm{~Hz}, 1 \mathrm{H}), 7.33(\mathrm{t}, J=7.2 \mathrm{~Hz}, 2 \mathrm{H})$, $7.27(\mathrm{~d}, J=7.4 \mathrm{~Hz}, 1 \mathrm{H}), 7.20(\mathrm{~d}, J=7.4 \mathrm{~Hz}, 2 \mathrm{H}), 6.89(\mathrm{~s}, 1 \mathrm{H}), 5.36(\mathrm{~s}, 2 \mathrm{H}), 4.43(\mathrm{q}, J=7.0 \mathrm{~Hz}$, 2H), 3.95 (s, 3H), 2.54 (s, 3H), 1.46 (t, $\left.J=7.1 \mathrm{~Hz}, 3 \mathrm{H}) ;{ }^{13} \mathrm{C} \mathrm{NMR} \mathrm{(101} \mathrm{MHz,} \mathrm{CDCl}_{3}\right) \delta 167.5$, 165.5, 148.1, 143.7, 136.2, 135.4, 132.8, 130.3, 129.0, 127.7, 126.0, 126.0, 123.7, 122.1, 115.9, 104.4, 100.3, 77.3, 77.0, 76.7, 61.4, 52.0, 47.7, 17.3, 14.3; HR-MS (ESI) calcd for $[\mathrm{M}+1]^{+}$: $\mathrm{C}_{24} \mathrm{H}_{23} \mathrm{~N}_{2} \mathrm{O}_{5}$ : 419.1601, found: 419.1605; IR (KBr): 2926, 1716, 1618, 1441, 1415, 1368, 1295 , $1249,1129,1055 \mathrm{~cm}^{-1}$.

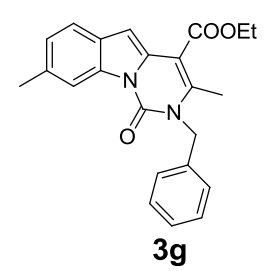

Ethyl 2-benzyl-3,8-dimethyl-1-oxo-1,2-dihydropyrimido[1,6-a]indole-4-carboxylate (3g) : White solid; $33.2 \mathrm{mg}, 89 \%$ yield; m.p. $165.4-166.0{ }^{\circ} \mathrm{C} .{ }^{1} \mathrm{H} \mathrm{NMR}\left(400 \mathrm{MHz}, \mathrm{CDCl}_{3}\right) \delta 8.48(\mathrm{~s}, 1 \mathrm{H})$, $7.53(\mathrm{~d}, J=8.0 \mathrm{~Hz}, 1 \mathrm{H}), 7.31(\mathrm{~d}, J=7.8 \mathrm{~Hz}, 2 \mathrm{H}), 7.27-7.23(\mathrm{~m}, 1 \mathrm{H}), 7.20(\mathrm{~d}, J=7.5 \mathrm{~Hz}, 3 \mathrm{H})$, $6.81(\mathrm{~s}, 1 \mathrm{H}), 5.35(\mathrm{~s}, 2 \mathrm{H}), 4.42(\mathrm{q}, J=7.1 \mathrm{~Hz}, 2 \mathrm{H}), 2.52(\mathrm{~s}, 6 \mathrm{H}), 1.45(\mathrm{t}, J=7.1 \mathrm{~Hz}, 3 \mathrm{H}) ;{ }^{13} \mathrm{C}$ NMR $\left(101 \mathrm{MHz}, \mathrm{CDCl}_{3}\right) \delta 165.9,148.4,142.3,136.6,133.4,132.8,131.0,129.0,128.4,127.5$, 126.0, 125.8, 119.4, 116.3, 104.7, 99.8, 61.3, 47.5, 21.9, 17.2, 14.3; HR-MS (ESI) calcd for [M + $1]^{+}: \mathrm{C}_{23} \mathrm{H}_{23} \mathrm{~N}_{2} \mathrm{O}_{3}$ : 375.1703, found: 375.1706; IR (KBr): 2922, 1688, 1594, 1493, 1410, 1367 , $1295,1243,1014 \mathrm{~cm}^{-1}$.

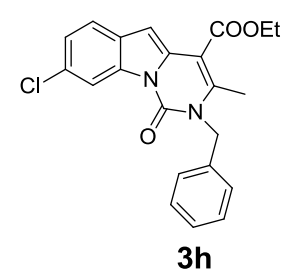

Ethyl 2-benzyl-8-chloro-3-methyl-1-oxo-1,2-dihydropyrimido[1,6-a]indole-4-carboxylate (3h) : Reddish solid; $25.6 \mathrm{mg}, 65 \%$ yield; m.p. $178.6-179.3{ }^{\circ} \mathrm{C} .{ }^{1} \mathrm{H}$ NMR (400 MHz, $\left.\mathrm{CDCl}_{3}\right) \delta$ $8.68(\mathrm{~s}, 1 \mathrm{H}), 7.52(\mathrm{t}, J=16.9 \mathrm{~Hz}, 1 \mathrm{H}), 7.36-7.31(\mathrm{~m}, 3 \mathrm{H}), 7.30-7.25(\mathrm{~m}, 1 \mathrm{H}), 7.20(\mathrm{~d}, J=7.3$ 
$\mathrm{Hz}, 2 \mathrm{H}), 6.82$ (s, 1H), 5.38 (s, 2H), 4.43 (q, $J=7.1 \mathrm{~Hz}, 2 \mathrm{H}), 2.56$ (s, 3H), 1.45 (t, $J=7.1 \mathrm{~Hz}, 3 \mathrm{H}$ ); ${ }^{13} \mathrm{C}$ NMR $\left(101 \mathrm{MHz}, \mathrm{CDCl}_{3}\right) \delta 165.5,148.1,143.3,136.3,133.2,132.2,129.0,128.4,127.7$, 126.0, 124.8, 120.5, 116.3, 104.5, 99.6, 61.4, 47.7, 17.3, 14.3; HR-MS (ESI) calcd for $[\mathrm{M}+1]^{+}$: $\mathrm{C}_{22} \mathrm{H}_{20} \mathrm{ClN}_{2} \mathrm{O}_{3}$ : 395.1157, found: 395.1161; IR (KBr): 2926, 1695, 1616, 1495, 1452, 1417, 1372 , 1316, 1235, $1055 \mathrm{~cm}$-1. IR (KBr): 2929, 1704, 1594, 1553, 1493, 1412, 1329, 1274, 1230, 1176 , $1054 \mathrm{~cm}^{-1}$.

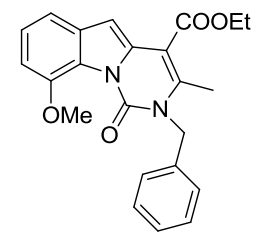

3i

Ethyl 2-benzyl-9-methoxy-3-methyl-1-oxo-1,2-dihydropyrimido[1,6-a]indole-4-carboxylate (3i) : White solid; $35.8 \mathrm{mg}, 92 \%$ yield; m.p. $160.8-161.6{ }^{\circ} \mathrm{C} .{ }^{1} \mathrm{H} \mathrm{NMR}\left(400 \mathrm{MHz}, \mathrm{CDCl}_{3}\right) \delta 7.30(\mathrm{t}$, $J=7.5 \mathrm{~Hz}, 3 \mathrm{H}), 7.27-7.21(\mathrm{~m}, 2 \mathrm{H}), 7.18(\mathrm{~d}, J=7.4 \mathrm{~Hz}, 2 \mathrm{H}), 6.88(\mathrm{~d}, J=8.5 \mathrm{~Hz}, 2 \mathrm{H}), 5.39(\mathrm{~s}$, $2 \mathrm{H}), 4.41(\mathrm{q}, J=7.1 \mathrm{~Hz}, 2 \mathrm{H}), 4.00(\mathrm{~s}, 2 \mathrm{H}), 2.51(\mathrm{~s}, 3 \mathrm{H}), 1.43(\mathrm{t}, J=7.1 \mathrm{~Hz}, 3 \mathrm{H}) ;{ }^{13} \mathrm{C}$ NMR $(101$ $\left.\mathrm{MHz}, \mathrm{CDCl}_{3}\right) \delta 165.8,149.2,147.5,143.4,136.8,134.1,133.2,128.9,127.4,126.1,125.5,122.7$, 113.1, 107.6, 104.3, 101.5, 61.2, 57.0, 48.2, 17.2, 14.3; HR-MS (ESI) calcd for $[\mathrm{M}+1]^{+}$: $\mathrm{C}_{23} \mathrm{H}_{23} \mathrm{~N}_{2} \mathrm{O}_{4}$ : 391.1652, found: 391.1652; IR (KBr): 2926, 1691, 1589, 1486, 1422, 1369, 1281, $1254,1138,1051 \mathrm{~cm}^{-1}$.

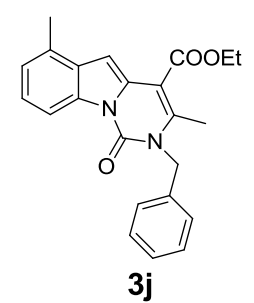

Ethyl 2-benzyl-3,6-dimethyl-1-oxo-1,2-dihydropyrimido[1,6-a]indole-4-carboxylate $(3 \mathbf{3 j})$ : White solid; $34.7 \mathrm{mg}, 93 \%$ yield; m.p. $157.0-157.5{ }^{\circ} \mathrm{C} .{ }^{1} \mathrm{H}$ NMR $\left(400 \mathrm{MHz}, \mathrm{CDCl}_{3}\right) \delta 8.48(\mathrm{~d}, J=$ $8.2 \mathrm{~Hz}, 1 \mathrm{H}), 7.34-7.29(\mathrm{~m}, 2 \mathrm{H}), 7.24(\mathrm{t}, J=5.2 \mathrm{~Hz}, 2 \mathrm{H}), 7.21-7.15(\mathrm{~m}, 3 \mathrm{H}), 6.88(\mathrm{~s}, 1 \mathrm{H}), 5.37$ (s, 2H), 4.44 (q, $J=7.1 \mathrm{~Hz}, 2 \mathrm{H}), 2.59$ (s, 3H), $2.52(\mathrm{~s}, 3 \mathrm{H}), 1.45(\mathrm{t}, J=7.1 \mathrm{~Hz}, 3 \mathrm{H}) ;{ }^{13} \mathrm{C}$ NMR $\left(101 \mathrm{MHz}, \mathrm{CDCl}_{3}\right) \delta 165.9,148.4,142.5,136.6,132.8,131.1,130.3,129.2,129.0,127.6,126.0$, 124.3, 122.9, 113.8, 104.7, 98.3, 61.3, 47.5, 18.6, 17.3, 14.3; HR-MS (ESI) calcd for $[\mathrm{M}+1]^{+}$: $\mathrm{C}_{23} \mathrm{H}_{23} \mathrm{~N}_{2} \mathrm{O}_{3}$ : 375.1703, found: 375.1706; IR (KBr): 2926, 1695, 1616, 1495, 1452, 1417, 1372 , $1316,1235,1055 \mathrm{~cm}^{-1}$. 


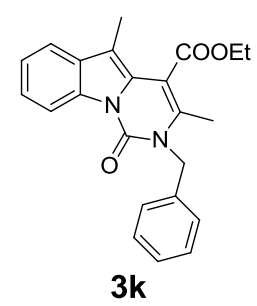

Ethyl 2-benzyl-3,5-dimethyl-1-oxo-1,2-dihydropyrimido[1,6-a]indole-4-carboxylate (3k) : White solid; $30.6 \mathrm{mg}, 82 \%$ yield; m.p. $151.8-152.4{ }^{\circ} \mathrm{C} .{ }^{1} \mathrm{H}$ NMR $\left(400 \mathrm{MHz}, \mathrm{CDCl}_{3}\right) \delta 8.64$ (d, J= $8.1 \mathrm{~Hz}, 1 \mathrm{H}), 7.60(\mathrm{~d}, J=7.5 \mathrm{~Hz}, 1 \mathrm{H}), 7.40-7.30(\mathrm{~m}, 4 \mathrm{H}), 7.23$ (dd, $J=14.4,4.8 \mathrm{~Hz}, 3 \mathrm{H}), 5.32$ (s, 2H), $4.41(\mathrm{q}, J=7.1 \mathrm{~Hz}, 2 \mathrm{H}), 2.28(\mathrm{~s}, 3 \mathrm{H}), 2.26(\mathrm{~s}, 3 \mathrm{H}), 1.40(\mathrm{t}, J=7.1 \mathrm{~Hz}, 3 \mathrm{H}) ;{ }^{13} \mathrm{C}$ NMR $(101$ $\left.\mathrm{MHz}, \mathrm{CDCl}_{3}\right) \delta 167.0,148.7,136.8,135.3,132.5,131.7,128.9,127.5,126.5,126.1,123.7,123.07$, $117.7,116.1,106.6,105.5,61.8,47.2,16.8,14.1,8.8$; HR-MS (ESI) calcd for $[\mathrm{M}+1]^{+}$: $\mathrm{C}_{23} \mathrm{H}_{23} \mathrm{~N}_{2} \mathrm{O}_{3}$ : 375.1703, found: 375.1701; IR (KBr): 2924, 1724, 1691, 1630, 1453, 1378, 1356, $1262,1133,1113 \mathrm{~cm}^{-1}$.

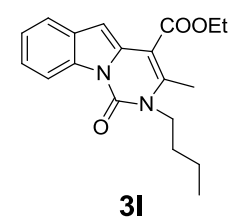

Ethyl 2-butyl-3-methyl-1-oxo-1,2-dihydropyrimido[1,6-a]indole-4-carboxylate (31) : White solid; $28.0 \mathrm{mg}, 86 \%$ yield; m.p. 99.7-100.3 ${ }^{\circ} \mathrm{C} .{ }^{1} \mathrm{H}$ NMR $\left(400 \mathrm{MHz}, \mathrm{CDCl}_{3}\right) \delta 8.63(\mathrm{~d}, J=7.8 \mathrm{~Hz}$, $1 \mathrm{H}), 7.63(\mathrm{~d}, J=7.1 \mathrm{~Hz}, 1 \mathrm{H}), 7.38-7.29(\mathrm{~m}, 2 \mathrm{H}), 6.81(\mathrm{~s}, 1 \mathrm{H}), 4.45(\mathrm{q}, J=7.1 \mathrm{~Hz}, 2 \mathrm{H}), 4.11-$ $4.03(\mathrm{~m}, 2 \mathrm{H}), 2.62(\mathrm{~s}, 3 \mathrm{H}), 1.72(\mathrm{dt}, J=15.5,7.8 \mathrm{~Hz}, 2 \mathrm{H}), 1.46(\mathrm{dd}, J=15.5,8.3 \mathrm{~Hz}, 5 \mathrm{H}), 1.00(\mathrm{t}$, $J=7.3 \mathrm{~Hz}, 3 \mathrm{H}) ;{ }^{13} \mathrm{C} \mathrm{NMR}\left(101 \mathrm{MHz}, \mathrm{CDCl}_{3}\right) \delta 166.0,147.8,142.5,132.8,131.7,130.6,124.0$, 122.5, 119.7, 116.1, 104.2, 99.2, 61.2, 44.6, 31.3, 20.1, 17.0, 14.3, 13.7; HR-MS (ESI) calcd for $[\mathrm{M}+1]^{+}: \mathrm{C}_{19} \mathrm{H}_{23} \mathrm{~N}_{2} \mathrm{O}_{3}: 327.1703$, found: 327.1704; IR (KBr): 2929, 1694, 1615, 1594, 1451, $1362,1316,1292,1180,1052 \mathrm{~cm}^{-1}$.

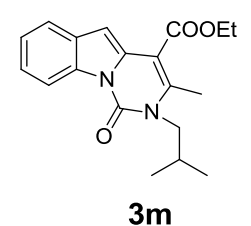

Ethyl 2-isobutyl-3-methyl-1-oxo-1,2-dihydropyrimido[1,6-a]indole-4-carboxylate (3m) : White solid; $27.4 \mathrm{mg}, 84 \%$ yield; m.p. 96.1-96.8 ${ }^{\circ} \mathrm{C} .{ }^{1} \mathrm{H}$ NMR $\left(400 \mathrm{MHz}, \mathrm{CDCl}_{3}\right) \delta 8.63(\mathrm{~d}, J=$ $7.8 \mathrm{~Hz}, 1 \mathrm{H}), 7.64(\mathrm{~d}, J=7.3 \mathrm{~Hz}, 1 \mathrm{H}), 7.39-7.30(\mathrm{~m}, 2 \mathrm{H}), 6.81(\mathrm{~s}, 1 \mathrm{H}), 4.45(\mathrm{q}, J=7.0 \mathrm{~Hz}, 2 \mathrm{H})$, $3.97(\mathrm{~d}, J=7.2 \mathrm{~Hz}, 2 \mathrm{H}), 2.61(\mathrm{~s}, 3 \mathrm{H}), 2.16(\mathrm{dt}, J=13.7,6.8 \mathrm{~Hz}, 1 \mathrm{H}), 1.47(\mathrm{t}, J=7.1 \mathrm{~Hz}, 3 \mathrm{H}), 0.99$ $(\mathrm{d}, J=6.6 \mathrm{~Hz}, 6 \mathrm{H}) ;{ }^{13} \mathrm{C}$ NMR $\left(101 \mathrm{MHz}, \mathrm{CDCl}_{3}\right) \delta 166.1,148.2,142.7,133.0,131.7,130.6$, 124.0, 122.5, 119.7, 116.2, 104.4, 99.3, 61.3, 50.9, 28.7, 20.0, 17.4, 14.3; HR-MS (ESI) calcd for 
$[\mathrm{M}+1]^{+}: \mathrm{C}_{19} \mathrm{H}_{23} \mathrm{~N}_{2} \mathrm{O}_{3}: 327.1703$, found: 327.1706; IR (KBr): 2927, 1695, 1615, 1594, 1451, $1362,1316,1261,1134,1051 \mathrm{~cm}^{-1}$.

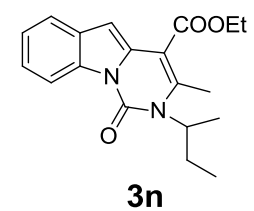

Ethyl 2-(sec-butyl)-3-methyl-1-oxo-1,2-dihydropyrimido[1,6-a]indole-4-carboxylate (3n) : White solid; $26.4 \mathrm{mg}, 81 \%$ yield; m.p. $98.4-98.9{ }^{\circ} \mathrm{C} .{ }^{1} \mathrm{H}$ NMR $\left(400 \mathrm{MHz}, \mathrm{CDCl}_{3}\right) \delta 8.63(\mathrm{~d}, J=$ $7.9 \mathrm{~Hz}, 1 \mathrm{H}), 7.63(\mathrm{~d}, J=7.3 \mathrm{~Hz}, 1 \mathrm{H}), 7.37-7.29(\mathrm{~m}, 2 \mathrm{H}), 6.74(\mathrm{~s}, 1 \mathrm{H}), 4.45$ (q, $J=7.0 \mathrm{~Hz}, 2 \mathrm{H})$, $4.31(\mathrm{~s}, 1 \mathrm{H}), 2.58(\mathrm{~s}, 3 \mathrm{H}), 2.42-2.29(\mathrm{~m}, 1 \mathrm{H}), 2.00(\mathrm{dt}, J=14.2,7.2 \mathrm{~Hz}, 1 \mathrm{H}), 1.64(\mathrm{~d}, J=6.7 \mathrm{~Hz}$, $3 \mathrm{H}), 1.47(\mathrm{t}, J=7.1 \mathrm{~Hz}, 3 \mathrm{H}), 0.91(\mathrm{t}, J=7.4 \mathrm{~Hz}, 3 \mathrm{H}) ;{ }^{13} \mathrm{C} \mathrm{NMR}\left(101 \mathrm{MHz}, \mathrm{CDCl}_{3}\right) \delta 166.4,142.6$, 132.9, 131.8, 130.5, 123.9, 122.5, 119.7, 116.2, 104.9, 98.7, 61.4, 18.5, 18.1, 14.3, 11.6; HR-MS (ESI) calcd for $[\mathrm{M}+1]^{+}: \mathrm{C}_{19} \mathrm{H}_{23} \mathrm{~N}_{2} \mathrm{O}_{3}: 327.1703$, found: 327.1706; IR (KBr): 2927, 1697, 1595, $1452,1417,1354,1292,1235,1188,1016 \mathrm{~cm}^{-1}$.

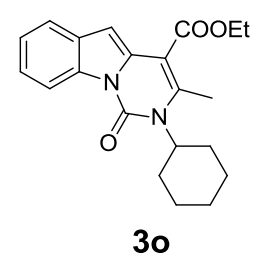

Ethyl 2-cyclohexyl-3-methyl-1-oxo-1,2-dihydropyrimido[1,6-a]indole-4-carboxylate (3o) : White solid; $29.2 \mathrm{mg}, 83 \%$ yield; m.p. 81.5-82.0 ${ }^{\circ} \mathrm{C} .{ }^{1} \mathrm{H}$ NMR $\left(400 \mathrm{MHz}, \mathrm{CDCl}_{3}\right) \delta 8.62$ (d, $J=$ $8.1 \mathrm{~Hz}, 1 \mathrm{H}), 7.62(\mathrm{~d}, J=7.6 \mathrm{~Hz}, 1 \mathrm{H}), 7.36-7.27(\mathrm{~m}, 2 \mathrm{H}), 6.73(\mathrm{~s}, 1 \mathrm{H}), 4.45(\mathrm{q}, J=7.1 \mathrm{~Hz}, 2 \mathrm{H})$, 4.09 (s, 1H), $2.74(\mathrm{~d}, J=10.4 \mathrm{~Hz}, 2 \mathrm{H}), 2.59(\mathrm{~s}, 3 \mathrm{H}), 1.93(\mathrm{~d}, J=5.1 \mathrm{~Hz}, 2 \mathrm{H}), 1.81-1.70(\mathrm{~m}, 3 \mathrm{H})$, $1.46(\mathrm{t}, J=7.1 \mathrm{~Hz}, 3 \mathrm{H}) ;{ }^{13} \mathrm{C} \mathrm{NMR}\left(101 \mathrm{MHz}, \mathrm{CDCl}_{3}\right) \delta 166.4,147.5,142.3,133.0,131.7,130.5$, 123.9, 122.48, 119.7, 116.2, 105.0, 98.7, 61.3, 59.8, 29.6, 26.6, 25.1, 18.0, 14.3; HR-MS (ESI) calcd for $[\mathrm{M}+1]^{+}: \mathrm{C}_{21} \mathrm{H}_{25} \mathrm{~N}_{2} \mathrm{O}_{3}: 353.1860$, found: 353.1865; IR (KBr): 2927, 1697, 1615, 1595, $1452,1416,1355,1234,1116,1014 \mathrm{~cm}^{-1}$.

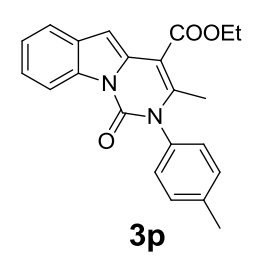

Ethyl 3-methyl-1-oxo-2-(p-tolyl)-1,2-dihydropyrimido[1,6-a]indole-4-carboxylate (3p) : White solid; $25.9 \mathrm{mg}, 72 \%$ yield; m.p. $171.1-171.8{ }^{\circ} \mathrm{C} .{ }^{1} \mathrm{H} \mathrm{NMR}\left(400 \mathrm{MHz}, \mathrm{CDCl}_{3}\right) \delta 8.55(\mathrm{~d}, J=$ $8.1 \mathrm{~Hz}, 1 \mathrm{H}), 7.66(\mathrm{~d}, J=7.7 \mathrm{~Hz}, 1 \mathrm{H}), 7.37-7.28(\mathrm{~m}, 4 \mathrm{H}), 7.18(\mathrm{~d}, J=8.1 \mathrm{~Hz}, 2 \mathrm{H}), 6.94(\mathrm{~s}, 1 \mathrm{H})$, 4.46 (q, $J=7.1 \mathrm{~Hz}, 2 \mathrm{H}), 2.44$ (s, 3H), 2.25 (s, 3H), 1.47 (t, $J=7.1 \mathrm{~Hz}, 3 \mathrm{H}) ;{ }^{13} \mathrm{C}$ NMR $(101 \mathrm{MHz}$, $\left.\mathrm{CDCl}_{3}\right) \delta 165.8,147.9,143.7,139.4,134.8,133.1,131.7,130.6,130.5,128.7,124.1,122.9,119.9$, 
116.1, 104.0, 100.3, 61.3, 21.2, 19.2, 14.3; HR-MS (ESI) calcd for $[\mathrm{M}+1]^{+}: \mathrm{C}_{22} \mathrm{H}_{21} \mathrm{~N}_{2} \mathrm{O}_{3}$ : 361.1547, found: 361.1549; IR (KBr): 2925, 1702, 1617, 1593, 1452, 1405, 1364, 1319, 1251, $1135,1016 \mathrm{~cm}^{-1}$.

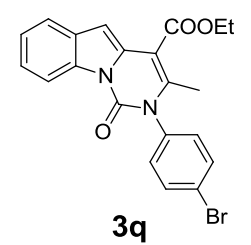

Ethyl 2-(4-bromophenyl)-3-methyl-1-oxo-1,2-dihydropyrimido[1,6-a]indole-4-carboxylate (3q) : Reddish solid; $25.8 \mathrm{mg}, 61 \%$ yield; m.p. 192.6-192.9 ${ }^{\circ} \mathrm{C} .{ }^{1} \mathrm{H}$ NMR $\left(400 \mathrm{MHz}, \mathrm{CDCl}_{3}\right) \delta$ $8.51(\mathrm{~d}, J=8.1 \mathrm{~Hz}, 1 \mathrm{H}), 7.67(\mathrm{t}, J=8.8 \mathrm{~Hz}, 3 \mathrm{H}), 7.33(\mathrm{ddd}, J=15.4,14.0,6.8 \mathrm{~Hz}, 2 \mathrm{H}), 7.19$ (d, $J$ $=8.5 \mathrm{~Hz}, 2 \mathrm{H}), 6.95(\mathrm{~s}, 1 \mathrm{H}), 4.46(\mathrm{q}, J=7.1 \mathrm{~Hz}, 2 \mathrm{H}), 2.22(\mathrm{~s}, 3 \mathrm{H}), 1.47(\mathrm{t}, J=7.1 \mathrm{~Hz}, 3 \mathrm{H}) ;{ }^{13} \mathrm{C}$ NMR (101 MHz, $\left.\mathrm{CDCl}_{3}\right) \delta 165.6,147.6,142.6,136.5,133.1,131.4,130.8,130.6,124.3,123.4$, $123.1,120.0,116.0,104.5,100.9,61.4,19.2,14.3$; HR-MS (ESI) calcd for $[\mathrm{M}+1]^{+}$: $\mathrm{C}_{21} \mathrm{H}_{18} \mathrm{BrN}_{2} \mathrm{O}_{3}$ : 425.0495, found: 425.0498; IR (KBr): 2924, 1688, 1452, 1370, 1274, 1183, 1140 $\mathrm{cm}^{-1}$.

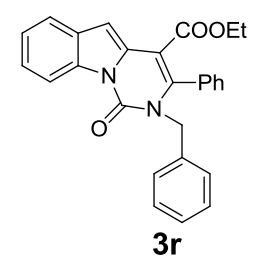

Ethyl 2-benzyl-1-oxo-3-phenyl-1,2-dihydropyrimido[1,6-a]indole-4-carboxylate (3r) : White solid; $31.7 \mathrm{mg}, 75 \%$ yield; m.p. 176.3-176.7 ${ }^{\circ} \mathrm{C} .{ }^{1} \mathrm{H}$ NMR $\left(400 \mathrm{MHz}, \mathrm{CDCl}_{3}\right) \delta 8.71(\mathrm{~d}, J=6.7 \mathrm{~Hz}$, 1H), $7.70(\mathrm{~d}, J=6.1 \mathrm{~Hz}, 1 \mathrm{H}), 7.40(\mathrm{~s}, 3 \mathrm{H}), 7.32(\mathrm{t}, J=6.7 \mathrm{~Hz}, 2 \mathrm{H}), 7.18(\mathrm{~s}, 3 \mathrm{H}), 7.12(\mathrm{~d}, J=6.8$ $\mathrm{Hz}, 2 \mathrm{H}), 7.03$ (s, 1H), 6.88 (s, 2H), 5.06 (s, 2H), 3.98 (dd, $J=12.4,5.9 \mathrm{~Hz}, 2 \mathrm{H}), 0.88$ (t, $J=6.1$ $\mathrm{Hz}, 3 \mathrm{H}) ;{ }^{13} \mathrm{C}$ NMR $\left(101 \mathrm{MHz}, \mathrm{CDCl}_{3}\right) \delta 164.8,148.3,143.9,137.0,133.5,133.3,131.6,130.7$, 129.3, 129.2, 128.4, 128.1, 127.3, 126.5, 124.2, 123.1, 120.1, 116.3, 106.1, 100.7, 60.9, 48.8, 13.5; HR-MS (ESI) calcd for $[\mathrm{M}+1]^{+}: \mathrm{C}_{27} \mathrm{H}_{23} \mathrm{~N}_{2} \mathrm{O}_{3}: 423.1703$, found: 423.1701; IR (KBr): 2926, 1695, $1593,1492,1449,1416,1355,1293,1228,1137,1026 \mathrm{~cm}^{-1}$.

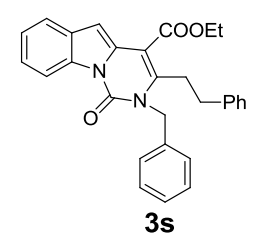

Ethyl 2-benzyl-1-oxo-3-phenethyl-1,2-dihydropyrimido[1,6-a]indole-4-carboxylate (3s) : White solid; $33.7 \mathrm{mg}, 75 \%$ yield; m.p. $183.2-183.7{ }^{\circ} \mathrm{C} .{ }^{1} \mathrm{H} \mathrm{NMR}\left(400 \mathrm{MHz}, \mathrm{CDCl}_{3}\right) \delta 8.66(\mathrm{~d}, J=$ $7.6 \mathrm{~Hz}, 1 \mathrm{H}), 8.66(\mathrm{~d}, J=7.6 \mathrm{~Hz}, 1 \mathrm{H}), 7.67(\mathrm{~d}, J=7.2 \mathrm{~Hz}, 1 \mathrm{H}), 7.34(\mathrm{dt}, J=31.8,10.8 \mathrm{~Hz}, 7 \mathrm{H})$, $7.88-7.06(\mathrm{~m}, 16 \mathrm{H}), 7.22(\mathrm{~s}, 1 \mathrm{H}), 7.18(\mathrm{t}, J=8.3 \mathrm{~Hz}, 4 \mathrm{H}), 6.94(\mathrm{~s}, 1 \mathrm{H}), 6.94(\mathrm{~s}, 1 \mathrm{H}), 5.41(\mathrm{~s}, 2 \mathrm{H})$, 
$4.44(\mathrm{q}, J=6.9 \mathrm{~Hz}, 2 \mathrm{H}), 3.20(\mathrm{~d}, J=6.9 \mathrm{~Hz}, 2 \mathrm{H}), 2.98-2.92(\mathrm{~m}, 2 \mathrm{H}), 1.46(\mathrm{t}, J=6.9 \mathrm{~Hz}, 3 \mathrm{H})$;

${ }^{13} \mathrm{C}$ NMR $\left(101 \mathrm{MHz}, \mathrm{CDCl}_{3}\right) \delta 165.4,148.5,145.9,140.0,136.7,133.1,131.5,130.7,129.0$, 128.7, 128.2, 127.6, 126.6, 125.9, 124.3, 122.9, 119.9, 116.3, 104.6, 100.6, 77.3, 77.0, 76.7, 61.4, 47.1, 36.0, 32.0, 14.4; HR-MS (ESI) calcd for $[\mathrm{M}+1]^{+}: \mathrm{C}_{29} \mathrm{H}_{26} \mathrm{~N}_{2} \mathrm{O}_{3}: 451.2016$, found: 451.2020; IR (KBr): 2927, 1694, 1593, 1495, 1451, 1412, 1370, 1317, 1290, 1121, $1028 \mathrm{~cm}^{-1}$.

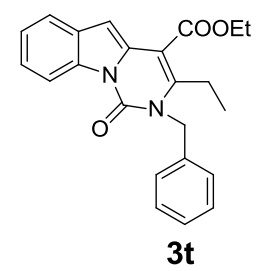

Ethyl 2-benzyl-3-ethyl-1-oxo-1,2-dihydropyrimido[1,6-a]indole-4-carboxylate (3t) : White solid; $29.2 \mathrm{mg}$, 78\% yield; m.p. 137.0-137.7 ${ }^{\circ} \mathrm{C} .{ }^{1} \mathrm{H}$ NMR (400 MHz, $\left.\mathrm{CDCl}_{3}\right) \delta 8.64(\mathrm{~d}, J=8.0 \mathrm{~Hz}$, $1 \mathrm{H}), 7.66(\mathrm{~d}, J=7.5 \mathrm{~Hz}, 1 \mathrm{H}), 7.39-7.30(\mathrm{~m}, 4 \mathrm{H}), 7.26(\mathrm{~d}, J=8.5 \mathrm{~Hz}, 1 \mathrm{H}), 7.18(\mathrm{~d}, J=7.4 \mathrm{~Hz}$, 2H), $6.90(\mathrm{~s}, 1 \mathrm{H}), 5.42(\mathrm{~s}, 2 \mathrm{H}), 4.45(\mathrm{q}, J=7.1 \mathrm{~Hz}, 2 \mathrm{H}), 2.91(\mathrm{~d}, J=7.0 \mathrm{~Hz}, 2 \mathrm{H}), 1.47$ (t, $J=7.1$ $\mathrm{Hz}, 3 \mathrm{H}), 1.32(\mathrm{t}, J=7.3 \mathrm{~Hz}, 3 \mathrm{H}) ;{ }^{13} \mathrm{C}$ NMR $\left(101 \mathrm{MHz}, \mathrm{CDCl}_{3}\right) \delta 165.5,148.4,147.8,136.8$, 133.1, 131.6, 130.6, 129.0, 127.6, 125.8, 124.2, 122.8, 119.8, 116.3, 104.0, 100.1, 61.3, 46.9, 23.5, 14.34, 14.1; HR-MS (ESI) calcd for [M + 1] $]^{+} \mathrm{C}_{23} \mathrm{H}_{23} \mathrm{~N}_{2} \mathrm{O}_{3}: 375.1703$, found: 375.1698 ; IR (KBr): 2927, 1694, 1593, 1451, 1414, 1370, 1290, 1173, 1125, $1026 \mathrm{~cm}^{-1}$.

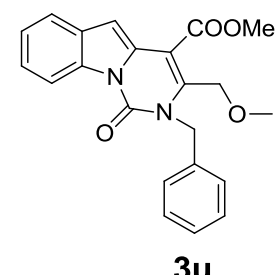

Methyl 2-benzyl-3-(methoxymethyl)-1-oxo-1,2-dihydropyrimido[1,6-a]indole-4-carboxylate (3u) : White oil; $34.3 \mathrm{mg}, 91 \%$ yield. ${ }^{1} \mathrm{H}$ NMR $\left(400 \mathrm{MHz}, \mathrm{CDCl}_{3}\right) \delta 8.68(\mathrm{~d}, J=5.9 \mathrm{~Hz}, 1 \mathrm{H}), 7.69$ (d, $J=5.0 \mathrm{~Hz}, 1 \mathrm{H}), 7.39$ (d, $J=4.0 \mathrm{~Hz}, 2 \mathrm{H}), 7.32(\mathrm{t}, J=7.0 \mathrm{~Hz}, 2 \mathrm{H}), 7.27(\mathrm{~d}, J=7.3 \mathrm{~Hz}, 1 \mathrm{H})$, $7.21(\mathrm{~d}, J=7.0 \mathrm{~Hz}, 2 \mathrm{H}), 6.92(\mathrm{~s}, 1 \mathrm{H}), 5.56(\mathrm{~s}, 2 \mathrm{H}), 4.57(\mathrm{~s}, 2 \mathrm{H}), 4.00(\mathrm{~s}, 3 \mathrm{H}), 3.43(\mathrm{~s}, 3 \mathrm{H}) ;{ }^{13} \mathrm{C}$ NMR $\left(101 \mathrm{MHz}, \mathrm{CDCl}_{3}\right) \delta 165.6,148.3,139.2,137.2,133.3,130.8,130.3,128.9,127.5,126.0$, 124.3, 123.4, 120.1, 116.4, 107.6, 101.7, 66.4, 58.1, 52.5, 47.0; HR-MS (ESI) calcd for $[\mathrm{M}+1]^{+}$: $\mathrm{C}_{22} \mathrm{H}_{20} \mathrm{~N}_{2} \mathrm{O}_{4}$ : 377.1496, found: 377.1498; IR (KBr): 2925, 1698, 1617, 1451, 1414, 1359, 1317 , $1238,1126,1032 \mathrm{~cm}^{-1}$.

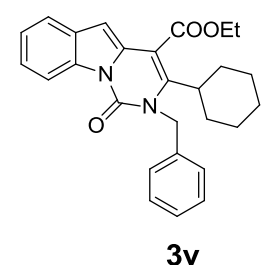


Ethyl 2-benzyl-3-cyclohexyl-1-oxo-1,2-dihydropyrimido[1,6-a]indole-4-carboxylate (3v) : White oil; $30.9 \mathrm{mg}, 72 \%$ yield. ${ }^{1} \mathrm{H}$ NMR $\left(400 \mathrm{MHz}, \mathrm{CDCl}_{3}\right) \delta 8.61(\mathrm{~d}, J=7.8 \mathrm{~Hz}, 1 \mathrm{H}), 7.62(\mathrm{~d}, J$ $=7.3 \mathrm{~Hz}, 1 \mathrm{H}), 7.33(\mathrm{t}, J=7.2 \mathrm{~Hz}, 4 \mathrm{H}), 7.26(\mathrm{~d}, J=8.1 \mathrm{~Hz}, 1 \mathrm{H}), 7.20(\mathrm{~d}, J=7.3 \mathrm{~Hz}, 2 \mathrm{H}), 6.51(\mathrm{~s}$, 1H), $5.41(\mathrm{~s}, 2 \mathrm{H}), 4.46(\mathrm{q}, J=7.0 \mathrm{~Hz}, 2 \mathrm{H}), 2.79(\mathrm{~s}, 1 \mathrm{H}), 1.75(\mathrm{~s}, 6 \mathrm{H}), 1.66(\mathrm{~d}, J=8.9 \mathrm{~Hz}, 1 \mathrm{H})$, $1.46(\mathrm{t}, J=7.1 \mathrm{~Hz}, 3 \mathrm{H}), 1.15(\mathrm{~d}, J=8.6 \mathrm{~Hz}, 3 \mathrm{H}) ;{ }^{13} \mathrm{C} \mathrm{NMR}\left(101 \mathrm{MHz}, \mathrm{CDCl}_{3}\right) \delta 166.7,148.7$, 137.3, 133.2, 132.2, 130.5, 128.9, 127.4, 125.8, 124.1, 122.7, 119.7, 116.2, 106.3, 97.7, 61.8, 47.5, 41.8, 30.8, 27.0, 25.7, 14.1; HR-MS (ESI) calcd for $[\mathrm{M}+1]^{+}: \mathrm{C}_{2} 7 \mathrm{H}_{29} \mathrm{~N}_{2} \mathrm{O}_{3}: 429.2173$, found: 429.2171; IR (KBr): 2928, 1726, 1693, 1620, 1495, 1450, 1374, 1291, 1118, $1023 \mathrm{~cm}^{-1}$.

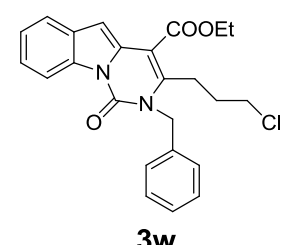

3w

Ethyl 2-benzyl-3-(3-chloropropyl)-1-oxo-1,2-dihydropyrimido[1,6-a]indole-4-carboxylate (3w) : White oil; $33.8 \mathrm{mg}, 80 \%$ yield. ${ }^{1} \mathrm{H}$ NMR $\left(400 \mathrm{MHz}, \mathrm{CDCl}_{3}\right) \delta 8.66(\mathrm{~d}, J=7.7 \mathrm{~Hz}, 1 \mathrm{H})$, $7.68-7.63(\mathrm{~m}, 1 \mathrm{H}), 7.40-7.30(\mathrm{~m}, 4 \mathrm{H}), 7.26(\mathrm{dd}, J=8.1,5.4 \mathrm{~Hz}, 1 \mathrm{H}), 7.21(\mathrm{~d}, J=7.2 \mathrm{~Hz}, 2 \mathrm{H})$, $6.92(\mathrm{~s}, 1 \mathrm{H}), 5.44(\mathrm{~s}, 2 \mathrm{H}), 4.44(\mathrm{q}, J=7.1 \mathrm{~Hz}, 2 \mathrm{H}), 3.63(\mathrm{t}, J=5.9 \mathrm{~Hz}, 2 \mathrm{H}), 3.08-3.00(\mathrm{~m}, 2 \mathrm{H})$, $2.15(\mathrm{td}, J=11.9,6.0 \mathrm{~Hz}, 2 \mathrm{H}), 1.47(\mathrm{t}, J=7.1 \mathrm{~Hz}, 3 \mathrm{H}) ;{ }^{13} \mathrm{C} \mathrm{NMR}\left(101 \mathrm{MHz}, \mathrm{CDCl}_{3}\right) \delta 165.4$, 148.4, 145.6, 136.6, 133.1, 131.3, 130.6, 129.0, 127.7, 126.0, 124.3, 123.0, 119.9, 116.3, 104.7, 100.6, 61.4, 46.9, 44.5, 32.4, 28.0, 14.3; HR-MS (ESI) calcd for $[\mathrm{M}+1]^{+}: \mathrm{C}_{24} \mathrm{H}_{24} \mathrm{ClN}_{2} \mathrm{O}_{3}$ : 423.1470, found: 423.1469; IR (KBr): 2923, 1691, 1605, 1538, 1469, 1365, 1154, $1056 \mathrm{~cm}^{-1}$.

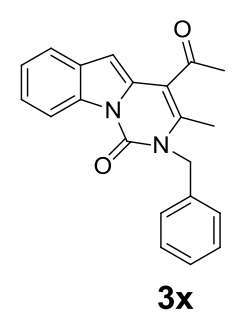

4-Acetyl-2-benzyl-3-methylpyrimido[1,6-a]indol-1(2H)-one (3x) : White solid; $18.8 \mathrm{mg}, 57 \%$ yield; m.p. 168.1-168.9 ${ }^{\circ} \mathrm{C} .{ }^{1} \mathrm{H}$ NMR $\left(400 \mathrm{MHz}, \mathrm{CDCl}_{3}\right) \delta 8.67$ (d, $\left.J=7.6 \mathrm{~Hz}, 1 \mathrm{H}\right), 7.65(\mathrm{~d}, J=$ $7.2 \mathrm{~Hz}, 1 \mathrm{H}), 7.36(\mathrm{dt}, J=21.2,7.1 \mathrm{~Hz}, 5 \mathrm{H}), 7.28(\mathrm{~d}, J=6.7 \mathrm{~Hz}, 2 \mathrm{H}), 7.22(\mathrm{~d}, J=7.4 \mathrm{~Hz}, 2 \mathrm{H})$, 6.52 (s, 1H), 5.37 (s, 2H), 2.63 (s, 3H), 2.33 (s, 3H); $\left.{ }^{13} \mathrm{C} \mathrm{NMR} \mathrm{(101} \mathrm{MHz,} \mathrm{CDCl}{ }_{3}\right) \delta 199.8,148.4$, 137.7, 136.5, 133.0, 131.5, 130.4, 129.0, 127.6, 126.1, 124.3, 123.0, 119.7, 116.3, 114.0, 98.1, 47.4, 31.5, 16.8; HR-MS (ESI) calcd for $[\mathrm{M}+1]^{+}: \mathrm{C}_{21} \mathrm{H}_{19} \mathrm{~N}_{2} \mathrm{O}_{2}: 331.1441$, found: 331.1444; IR (KBr): 2927, 1731, 1695, 1593, 1478, 1369, 1311, 1253, 1128, $1057 \mathrm{~cm}^{-1}$. 


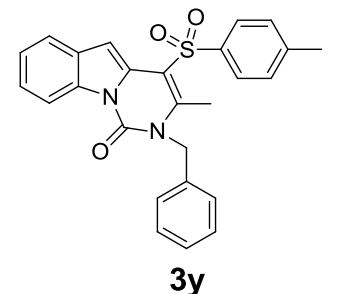

$3 y$

2-Benzyl-3-methyl-4-tosylpyrimido[1,6-a]indol-1(2H)-one (3y) : yellow solid; $35.8 \mathrm{mg}, 81 \%$ yield; m.p. 190.5-191.0 ${ }^{\circ} \mathrm{C} .{ }^{1} \mathrm{H}$ NMR $\left(400 \mathrm{MHz}, \mathrm{CDCl}_{3}\right) \delta 8.60(\mathrm{~d}, J=7.6 \mathrm{~Hz}, 1 \mathrm{H}), 7.84(\mathrm{~d}, J=$ $7.5 \mathrm{~Hz}, 2 \mathrm{H}), 7.71(\mathrm{~d}, J=7.2 \mathrm{~Hz}, 1 \mathrm{H}), 7.47$ (s, 1H), $7.42-7.24(\mathrm{~m}, 9 \mathrm{H}), 7.15$ (d, $J=6.9 \mathrm{~Hz}, 2 \mathrm{H})$, 5.37 (s, 2H), 2.72 (s, 3H), 2.38 (s, 3H); ${ }^{13} \mathrm{C} \mathrm{NMR}\left(101 \mathrm{MHz}, \mathrm{CDCl}_{3}\right) \delta$ 147.7, 144.4, 144.1, 139.5, 135.9, 133.0, 130.5, 129.8, 129.1, 129.0, 127.8, 126.3, 125.9, 124.5, 123.5, 120.4, 116.2, 113.6, 102.2, 47.8, 21.5, 16.7; HR-MS (ESI) calcd for $[\mathrm{M}+1]^{+}: \mathrm{C}_{26} \mathrm{H}_{23} \mathrm{~N}_{2} \mathrm{O}_{3} \mathrm{~S}: 443.1424$, found: 443.1433; IR (KBr): 2923, 1702, 1591, 1494, 1451, 1360, 1315, 1249, 1132, $1083 \mathrm{~cm}^{-1}$.<smiles>CCOC(=O)c1c(C)n(Cc2ccccc2)c(=O)n2cccc12</smiles>

Ethyl 2-benzyl-3-methyl-1-oxo-1,2-dihydropyrrolo[1,2-c]pyrimidine-4-carboxylate $(3 z)$ : yellow oil; $14.6 \mathrm{mg}, 47 \%$ yield. ${ }^{1} \mathrm{H}$ NMR $\left(400 \mathrm{MHz}, \mathrm{CDCl}_{3}\right) \delta 7.63(\mathrm{~s}, 1 \mathrm{H}), 7.33(\mathrm{t}, J=7.5 \mathrm{~Hz}$, 2H), $7.27(\mathrm{~d}, J=8.8 \mathrm{~Hz}, 1 \mathrm{H}), 7.18(\mathrm{~d}, J=7.5 \mathrm{~Hz}, 2 \mathrm{H}), 6.67(\mathrm{t}, J=2.9 \mathrm{~Hz}, 1 \mathrm{H}), 6.62(\mathrm{~s}, 1 \mathrm{H}), 5.39$ $(\mathrm{s}, 2 \mathrm{H}), 4.40(\mathrm{q}, J=7.1 \mathrm{~Hz}, 2 \mathrm{H}), 2.55(\mathrm{~s}, 3 \mathrm{H}), 1.42(\mathrm{t}, J=7.1 \mathrm{~Hz}, 3 \mathrm{H}) ;{ }^{13} \mathrm{C}$ NMR $(101 \mathrm{MHz}$, $\left.\mathrm{CDCl}_{3}\right) \delta 165.8,147.3,139.1,136.3,128.9,127.6,126.1,115.1,115.0,105.9,105.4,61.2,48.0$, 16.7, 14.2; HR-MS (ESI) calcd for $[\mathrm{M}+1]^{+}: \mathrm{C}_{18} \mathrm{H}_{19} \mathrm{~N}_{2} \mathrm{O}_{3}: 311.1390$, found: 311.1397; IR (KBr): 2924, 1694, 1604, 1434, 1374, 1343, 1310, 1241, 1153, $1097 \mathrm{~cm}^{-1}$.

\section{Synthetic Application for This Transformation}

2.1 Procedure for the synthesis of ethyl 3-methyl-1-oxo-1,2-dihydropyrimido[1,6-a]indole-4carboxylate (3a-1) ${ }^{[3]}$

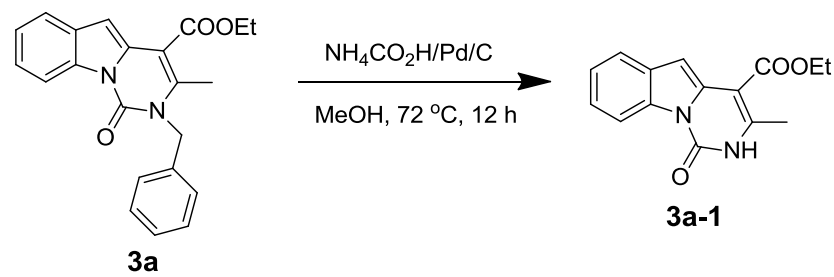

A mixture of compound $\mathbf{3 a}(36 \mathrm{mg}, 0.1 \mathrm{mmol})$, ammonium formate $(3.3 \mathrm{~mL}$ of a $0.4 \mathrm{~N}$ solution in 
dry $\mathrm{MeOH}$ ) and $10 \%$ palladium_charcoal $(77 \mathrm{mg} 10 \% \mathrm{Pd} / \mathrm{C}, 0.072 \mathrm{mmol}$ of $\mathrm{Pd}, 0.55$ equiv of $\mathrm{Pd})$ was refluxed at $72{ }^{\circ} \mathrm{C}$ for $12 \mathrm{~h}$. The corresponding reaction mixture was cooled to room temperature and then filtered through Celite, and the solid residue was extensively washed with $\mathrm{MeOH}$ and $\mathrm{CHCl}_{3}$. Removal of solvents under reduced pressure and following column chromatography to give the pure free N-H product 3a-1.

Ethyl 3-methyl-1-oxo-1,2-dihydropyrimido[1,6-a]indole-4-carboxylate (3a-1) : White solid; $23.2 \mathrm{mg}, 86 \%$ yield; m.p. $134.8-135.6{ }^{\circ} \mathrm{C} .{ }^{1} \mathrm{H}$ NMR (400 MHz, DMSO) $\delta 11.36$ (s, $\left.1 \mathrm{H}\right), 8.48$ (d, $J$ $=8.0 \mathrm{~Hz}, 1 \mathrm{H}), 7.68(\mathrm{~d}, J=7.7 \mathrm{~Hz}, 1 \mathrm{H}), 7.36-7.27(\mathrm{~m}, 2 \mathrm{H}), 6.98(\mathrm{~s}, 1 \mathrm{H}), 4.33(\mathrm{q}, J=7.1 \mathrm{~Hz}, 2 \mathrm{H})$, 2.50 (s, 3H), 1.37 (t, $J=7.1 \mathrm{~Hz}, 3 \mathrm{H}) .{ }^{13} \mathrm{C}$ NMR (101 MHz, DMSO) $\delta 164.5,146.7,145.6,133.16$, 131.7, 130.3, 123.7, 121.9, 119.7, 115.1, 99.7, 99.1, 60.3, 18.2, 14.1; HR-MS (ESI) calcd for [M + $1]^{+}: \mathrm{C}_{15} \mathrm{H}_{15} \mathrm{~N}_{2} \mathrm{O}_{3}: 271.0251$, found: 271.0254; IR (KBr): 3402, 2924, 1701, 1635, 1583, 14768 , $1365,1301,1233,1130,1051 \mathrm{~cm}^{-1}$.

2.2 Procedure for the synthesis of 2-benzyl-4-(hydroxymethyl)-3-methylpyrimido[1,6a]indol-1(2H)-one (3a-2) ${ }^{[4]}$

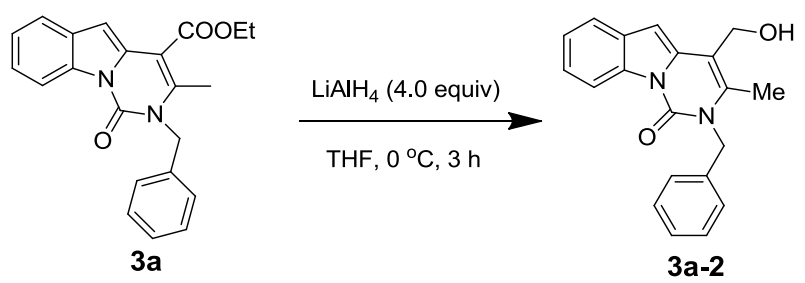

A solution of $3 \mathbf{a}(36 \mathrm{mg}, 0.1 \mathrm{mmol})$ in THF $(5 \mathrm{~mL})$ was cooled to $0{ }^{\circ} \mathrm{C}$. $\mathrm{LiAlH}_{4}(0.4 \mathrm{~mL}, 0.4$ mmol, $1 \mathrm{M}$ in THF) was added dropwise, and the reaction mixture was stirred at room temperature for $3 \mathrm{~h}$. The reaction was quenched by the dropwise addition of absolute ethanol followed by saturated aqueous $\mathrm{NaHCO}_{3}$. The precipitate was removed by filtration and then washed with EtOAc. The aqueous layer was extracted with EtOAc $(3 \times 10 \mathrm{~mL})$. The combined organic layers were washed with brine, dried over $\mathrm{MgSO}_{4}$, filtered and concentrated. The residue was purified by flash chromatography on silical gel using ethyl acetate/petroleum ether as eluent to afford the desired product 3a-2.

2-Benzyl-4-(hydroxymethyl)-3-methylpyrimido[1,6-a]indol-1(2H)-one (3a-2) : White solid; $26.1 \mathrm{mg}, 82 \%$ yield; m.p. 174.1-174.59 ${ }^{\circ} \mathrm{C} .{ }^{1} \mathrm{H}$ NMR (400 MHz, DMSO) $\delta 8.54(\mathrm{~d}, J=8.2 \mathrm{~Hz}$, 1H), 7.69 (d, $J=7.8 \mathrm{~Hz}, 1 \mathrm{H}), 7.38-7.26(\mathrm{~m}, 6 \mathrm{H}), 7.23$ (d, $J=7.6 \mathrm{~Hz}, 2 \mathrm{H}), 6.72(\mathrm{~s}, 1 \mathrm{H}), 5.35$ (s, 2H), $5.04(\mathrm{t}, J=5.0 \mathrm{~Hz}, 1 \mathrm{H}), 4.54(\mathrm{~d}, J=4.8 \mathrm{~Hz}, 2 \mathrm{H}), 2.29(\mathrm{~s}, 3 \mathrm{H}) ;{ }^{13} \mathrm{C}$ NMR (101 MHz, DMSO) $\delta 148.4,137.7,135.9,135.6,132.4,130.3,128.7,127.1,125.9,123.6,121.6,119.5,115.5,109.0$, 95.3, 56.8, 46.6, 14.9; HR-MS (ESI) calcd for $[\mathrm{M}+1]^{+}: \mathrm{C}_{20} \mathrm{H}_{19} \mathrm{~N}_{2} \mathrm{O}_{2}: 319.1441$, found: 319.1442; IR (KBr): 3458, 2924, 1692, 1618, 1492, 1374, 1358, 1283, 1113, $1048 \mathrm{~cm}^{-1}$.

2.3 Procedure for the synthesis of 2-benzyl-3-methylpyrimido[1,6-a]indol-1(2H)-one (3a-3) ${ }^{[5]}$ 


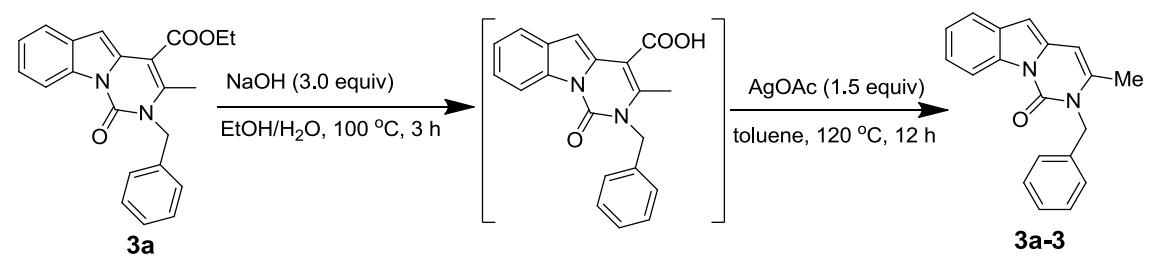

3a $(36 \mathrm{mg}, 0.1 \mathrm{mmol})$ was dissolved in $\mathrm{EtOH}(2 \mathrm{~mL})$ and aqueous $1 \mathrm{~N} \mathrm{NaOH}(0.3 \mathrm{~mL})$ were added, and the reaction mixture was heated at reflux for $3.5 \mathrm{~h}$. The mixture was cooled to room temperature and transferred to a separatory funnel containing saturated aqueous $\mathrm{NaHCO}_{3}(15 \mathrm{~mL})$. The aqueous layer was extracted with EtOAc $(3 \times 15 \mathrm{~mL})$. The combined organic layers were washed with brine, dried over $\mathrm{MgSO}_{4}$, filtered and concentrated to give the crude carboxylic acid product. The crude solid was dissolved in toluene $(1 \mathrm{~mL})$ and then $\mathrm{AgOAc}$ (1.5 equiv) was added. The reaction mixture was stirred at $120{ }^{\circ} \mathrm{C}$ for $12 \mathrm{~h}$. The corresponding reaction mixture was cooled to room temperature and then filtered through a pad of Celite, washed wih EtOAc and concentrated. The residue was purified by flash chromatography on silical gel using ethyl acetate/petroleum ether as eluent to afford the decarbonylated product 3a-3.

2-Benzyl-3-methylpyrimido[1,6-a]indol-1(2H)-one (3a-3) : White solid; $19.9 \mathrm{mg}$, 69\% yield; m.p. 154.2-154.7 ${ }^{\circ} \mathrm{C} .{ }^{1} \mathrm{H}$ NMR $\left(400 \mathrm{MHz}, \mathrm{CDCl}_{3}\right) \delta 8.67(\mathrm{~d}, J=7.9 \mathrm{~Hz}, 1 \mathrm{H}), 7.61(\mathrm{~d}, J=7.2 \mathrm{~Hz}$, 1H), $7.37-7.26(\mathrm{~m}, 5 \mathrm{H}), 7.21$ (d, J=7.3 Hz, 2H), 6.40 (s, 1H), 6.25 (s, 1H), 5.30 (s, 2H), 2.25 (s, $3 \mathrm{H}) ;{ }^{13} \mathrm{C} \mathrm{NMR}\left(101 \mathrm{MHz}, \mathrm{CDCl}_{3}\right) \delta 149.5,137.2,136.2,134.4,133.2,130.8,128.9,127.4,126.1$, 123.8, 122.0, 119.4, 116.1, 99.0, 96.1, 47.0, 20.0; HR-MS (ESI) calcd for $[\mathrm{M}+1]^{+}: \mathrm{C}_{19} \mathrm{H}_{17} \mathrm{~N}_{2} \mathrm{O}$ : 288.1155, found: 288.1159; IR (KBr): 2922, 1698, 1618, 1485, 1448, 1374, 1278, $1051 \mathrm{~cm}^{-1}$.

\subsection{Procedure for the synthesis of ethyl 2-benzyl-3-methyl-1-oxo-1,2,3,4-} tetrahydropyrimido[1,6-a]indole-4-carboxylate $(3 \mathrm{a}-4)^{[6]}$

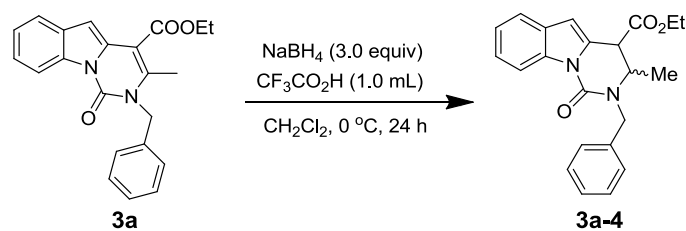

$\mathrm{NaBH}_{4}(11.4 \mathrm{mg}, 0.3 \mathrm{mmol})$ was added to $\mathrm{CF}_{3} \mathrm{COOH}(1.0 \mathrm{~mL})$ in portion at $0{ }^{\circ} \mathrm{C}$ under argon. To the mixture was added dropwise a solution $3 \mathbf{a}(36 \mathrm{mg}, 0.1 \mathrm{mmol})$ in dry DCM $(1 \mathrm{~mL})$ at $0{ }^{\circ} \mathrm{C}$. The reaction mixture was then stirred at $25{ }^{\circ} \mathrm{C}$ for $24 \mathrm{~h}$. Upon the completion of the reaction as determined by TLC, the reaction was quenched by $\mathrm{H}_{2} \mathrm{O}$, basified with a $1 \mathrm{M}$ aqueous $\mathrm{NaOH}$ solution and extracted with DCM $(3 \times 10 \mathrm{~mL})$. The combined organic layers were dried over $\mathrm{MgSO}_{4}$, filtered and concentrated. The product was purified by flash column chromatography on silica gel to give the corresponding product 3a-4. The ratio of cis/trans isomers of 3a-4 (Z/E=1:1) was determined by ${ }^{1} \mathrm{H}$ NMR spectrum.

Ethyl 2-benzyl-3-methyl-1-oxo-1,2,3,4-tetrahydropyrimido[1,6-a]indole-4-carboxylate (3a-4) : White solid; $29.3 \mathrm{mg}, 81 \%$ yield; m.p. $139.1-139.7{ }^{\circ} \mathrm{C} .{ }^{1} \mathrm{H} \mathrm{NMR}\left(400 \mathrm{MHz}, \mathrm{CDCl}_{3}\right) \delta 8.43(\mathrm{~d}, J=$ 
$8.3 \mathrm{~Hz}, 1 \mathrm{H}), 7.54$ (d, $J=7.8 \mathrm{~Hz}, 1 \mathrm{H}), 7.34$ (ddd, $J=19.3,12.4,6.9 \mathrm{~Hz}, 6 \mathrm{H}), 7.23$ (t, $J=7.5 \mathrm{~Hz}$, 1H), 6.49 (s, 1H), $5.12(\mathrm{~s}, 1 \mathrm{H}), 5.09(\mathrm{~s}, 1 \mathrm{H}), 4.32(\mathrm{~s}, 1 \mathrm{H}), 4.28(\mathrm{~s}, 0 \mathrm{H}), 4.07$ (q, $J=6.4 \mathrm{~Hz}, 1 \mathrm{H})$, $3.96-3.89(\mathrm{~m}, 1 \mathrm{H}), 3.83-3.76(\mathrm{~m}, 2 \mathrm{H}), 1.14(\mathrm{~d}, J=6.7 \mathrm{~Hz}, 3 \mathrm{H}), 1.02(\mathrm{t}, J=7.1 \mathrm{~Hz}, 3 \mathrm{H}),{ }^{13} \mathrm{C}$ NMR $\left(101 \mathrm{MHz}, \mathrm{CDCl}_{3}\right) \delta 169.4,150.1,137.2,135.6,129.9,128.9,128.7,128.6,127.7,124.2$, 122.6, 120.2, 115.4, 106.7, 61.7, 53.4, 49.6, 46.0, 18.5, 13.8; HR-MS (ESI) calcd for $[\mathrm{M}+1]^{+}$: $\mathrm{C}_{22} \mathrm{H}_{23} \mathrm{~N}_{2} \mathrm{O}_{3}$ : 362.1523, found: 362.1525; IR (KBr): 2927, 1702, 1693, 1583, 1495, 1421, 1378, $1338,1132,1041 \mathrm{~cm}^{-1}$.

\section{Controlled experiments for mechanism studies}

(a) H/D Exchange of $N$-carbamoyl indole (1k)
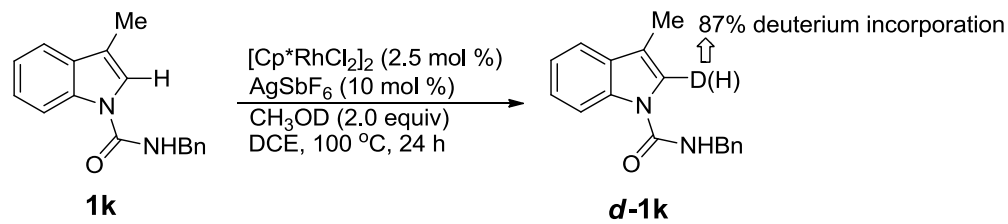

To the solution of $N$-carbamoyl indole 1a $(26.4 \mathrm{mg}, 0.1 \mathrm{mmol})$ in DCE $(1.0 \mathrm{~mL})$ were added $\left[\mathrm{Cp}^{*} \mathrm{RhCl}_{2}\right]_{2}(1.6 \mathrm{mg}, 2.5 \mathrm{~mol} \%), \mathrm{AgSbF}_{6}(5.1 \mathrm{mg}, 15 \mathrm{~mol} \%)$ and $\mathrm{CD}_{3} \mathrm{OD}$ (2.0 equiv) under Ar. The reaction mixture was stirred at $100{ }^{\circ} \mathrm{C}$ for $24 \mathrm{~h}$ and then cooled down to room temperature. After removal of the solvent, the resulted crude product was quickly purified by flash column chromatography to give the desired compound $\boldsymbol{d}-\mathbf{1 k}$ (96\% yield) as white solid. The deuterium incorporation was determined to be $87 \%$ at the C-2 position by ${ }^{1} \mathrm{H}$ NMR method (Figure 1). ${ }^{1} \mathrm{H}$ NMR $\left(400 \mathrm{MHz}, \mathrm{CDCl}_{3}\right) \delta 8.11(\mathrm{~d}, J=8.2 \mathrm{~Hz}, 1 \mathrm{H}), 7.51(\mathrm{~d}, J=7.7 \mathrm{~Hz}, 1 \mathrm{H}), 7.35(\mathrm{~d}, J=3.3 \mathrm{~Hz}$, 4H), $7.30(\mathrm{dd}, J=12.0,4.9 \mathrm{~Hz}, 2 \mathrm{H}), 7.25-7.20(\mathrm{~m}, 1 \mathrm{H}), 7.18(\mathrm{~s}, 0.13 \mathrm{H}), 5.85(\mathrm{~s}, 1 \mathrm{H}), 4.60(\mathrm{~d}, J$ $=5.5 \mathrm{~Hz}, 2 \mathrm{H}), 2.25(\mathrm{~s}, 3 \mathrm{H}) ;{ }^{13} \mathrm{C} \mathrm{NMR}\left(101 \mathrm{MHz}, \mathrm{CDCl}_{3}\right) \delta 152.1,137.9,135.5,130.9,128.8$, $127.8,127.7,124.3,122.0,120.9,119.1,116.4,114.3,44.8,9.5$.

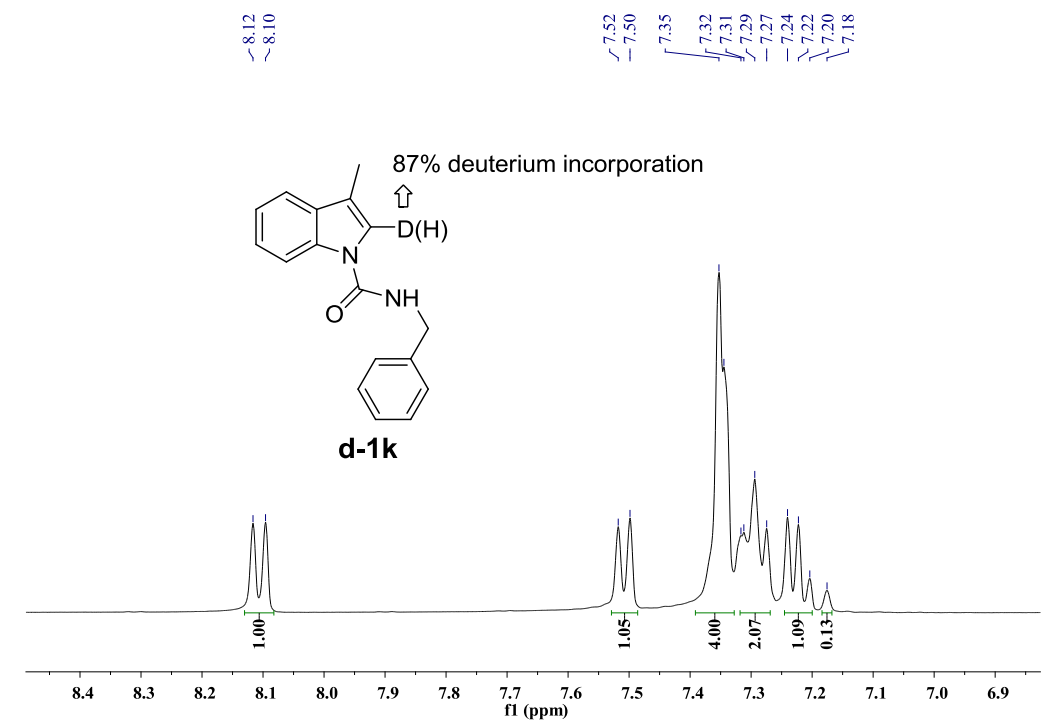

Figure 1. The ${ }^{1} \mathrm{H}$ NMR spectra of $\boldsymbol{d}-\mathbf{- 1 a}$ 
(b): H/D Exchange of $N$-acetyl indole (1t)<smiles></smiles>

$1 \mathbf{t}$
$\left[\mathrm{Cp}^{*} \mathrm{RhCl}_{2}\right]_{2}(2.5 \mathrm{~mol} \%)$ $\mathrm{AgSbF}_{6}(10 \mathrm{~mol} \%)$ $\mathrm{CH}_{3} \mathrm{OD}$ (2.0 equiv) DCE, $100^{\circ} \mathrm{C}, 24 \mathrm{~h}$

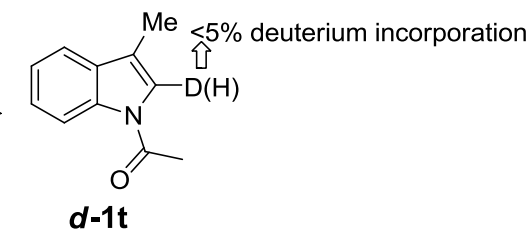

To the solution of $N$-acetyl indole $1 \mathrm{t}(26.4 \mathrm{mg}, 0.1 \mathrm{mmol})$ in DCE $(1.0 \mathrm{~mL})$ were added $\left[\mathrm{Cp}^{*} \mathrm{RhCl}_{2}\right]_{2}(1.6 \mathrm{mg}, 2.5 \mathrm{~mol} \%), \mathrm{AgSbF}_{6}(5.1 \mathrm{mg}, 15 \mathrm{~mol} \%)$ and $\mathrm{CD}_{3} \mathrm{OD}$ (2.0 equiv) under Ar. The reaction mixture was stirred at $100{ }^{\circ} \mathrm{C}$ for $24 \mathrm{~h}$ and then cooled down to room temperature. After removal of the solvent, the resulted crude product was quickly purified by flash column chromatography to give the desired compound $\boldsymbol{d}$-1t (97\% yield) as white oil. The deuterium incorporation was determined to be $<5 \%$ at the $\mathrm{C}-2$ position by ${ }^{1} \mathrm{H}$ NMR method (Figure 2). ${ }^{1} \mathrm{H}$ $\operatorname{NMR}\left(400 \mathrm{MHz}, \mathrm{CDCl}_{3}\right) \delta 8.41(\mathrm{~d}, J=6.5 \mathrm{~Hz}, 1 \mathrm{H}), 7.49(\mathrm{~d}, J=7.5 \mathrm{~Hz}, 1 \mathrm{H}), 7.37-7.32(\mathrm{~m}, 1 \mathrm{H})$, $7.29(\mathrm{dd}, J=10.8,4.1 \mathrm{~Hz}, 1 \mathrm{H}), 7.16(\mathrm{~s}, 1 \mathrm{H}), 2.58(\mathrm{~s}, 3 \mathrm{H}), 2.28(\mathrm{~s}, 3 \mathrm{H}) ;{ }^{13} \mathrm{C}$ NMR $(101 \mathrm{MHz}$, $\left.\mathrm{CDCl}_{3}\right) \delta 168.2,135.8,131.4,125.1,123.3,122.2,118.8,118.3,116.5,23.9,9.6$.
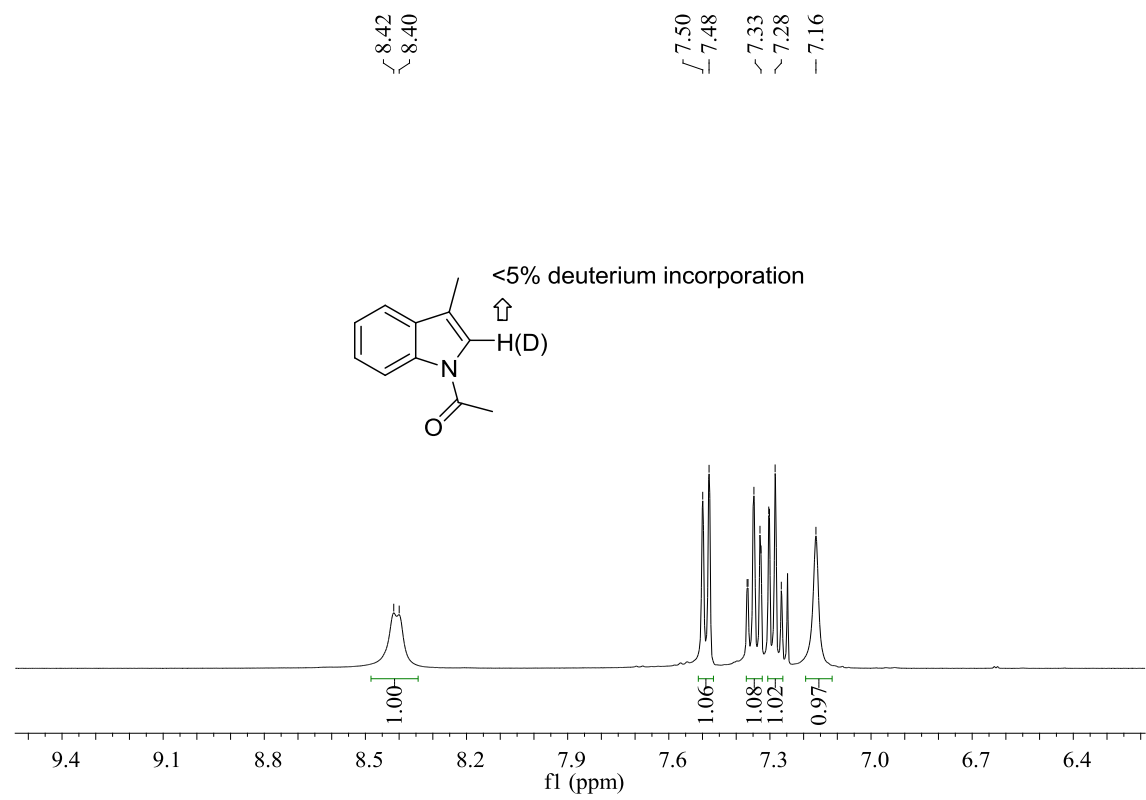

Figure 2. The ${ }^{1} \mathrm{H}$ NMR spectra of $\boldsymbol{d}$-1t

(c): Kinetic isotope effect of the [4 + 2] annulation of indole (1k) with diazo compound (2a). 
<smiles>Cc1c(CNCc2ccccc2)n(C(=O)NCc2ccccc2)c2ccccc12</smiles>

$1 \mathrm{k}$

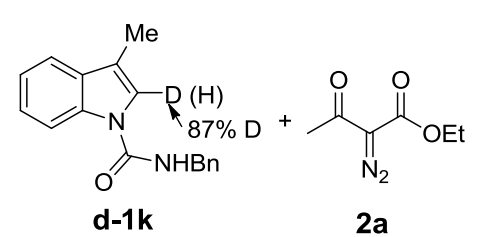

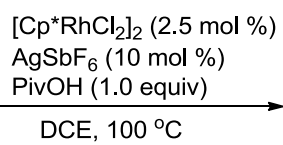

DCE, $100^{\circ} \mathrm{C}$

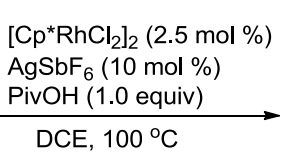

DCE, $100^{\circ} \mathrm{C}$

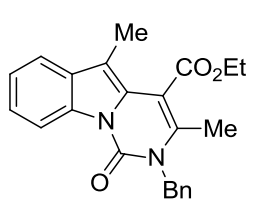

3k

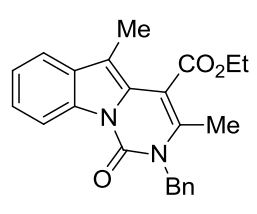

3k

A $10 \mathrm{~mL}$ of reaction tube was charged with $N$-carbamoyl indole (1k: $26.4 \mathrm{mg}, 0.1 \mathrm{mmol}$; or $\boldsymbol{d}$-1k: $26.4 \mathrm{mg}, 0.1 \mathrm{mmol}$ ), [Cp* $\left.\mathrm{RhCl}_{2}\right]_{2}(1.6 \mathrm{mg}, 2.5 \mathrm{~mol} \%), \mathrm{AgSbF}_{6}$ (3.4 mg, $\left.10 \mathrm{~mol} \%\right)$, PivOH (10.2 $\mathrm{mg}, 1$ equiv) and DCE (2.0 mL) under Ar. Diazo compound 2a (31 mg, $0.2 \mathrm{mmol})$ in DCE $(0.5$ $\mathrm{mL}$ ) was then added in one-pot under $\mathrm{Ar}$ and the mixture was stirred at $100{ }^{\circ} \mathrm{C}$. Aliquots $(0.4 \mathrm{~mL})$ were extracted at 2 hour intervals for the first 8 hours of the reaction. After the solvent of each aliquot $(0.4 \mathrm{~mL})$ was removed under reduced pressure conditions and analyzed by ${ }^{1} \mathrm{H}$ NMR spectrum (see Figure 3 and Figure 4). A sample plot of the initial rate data for the reactionn of both $1 \mathbf{k}$ and $\boldsymbol{d}$-1k was shown in Figure $\mathbf{5}$. The reaction progress in the early stage (0-8 hours) indicated a kinetic isotope effect (KIE) of 2.0.

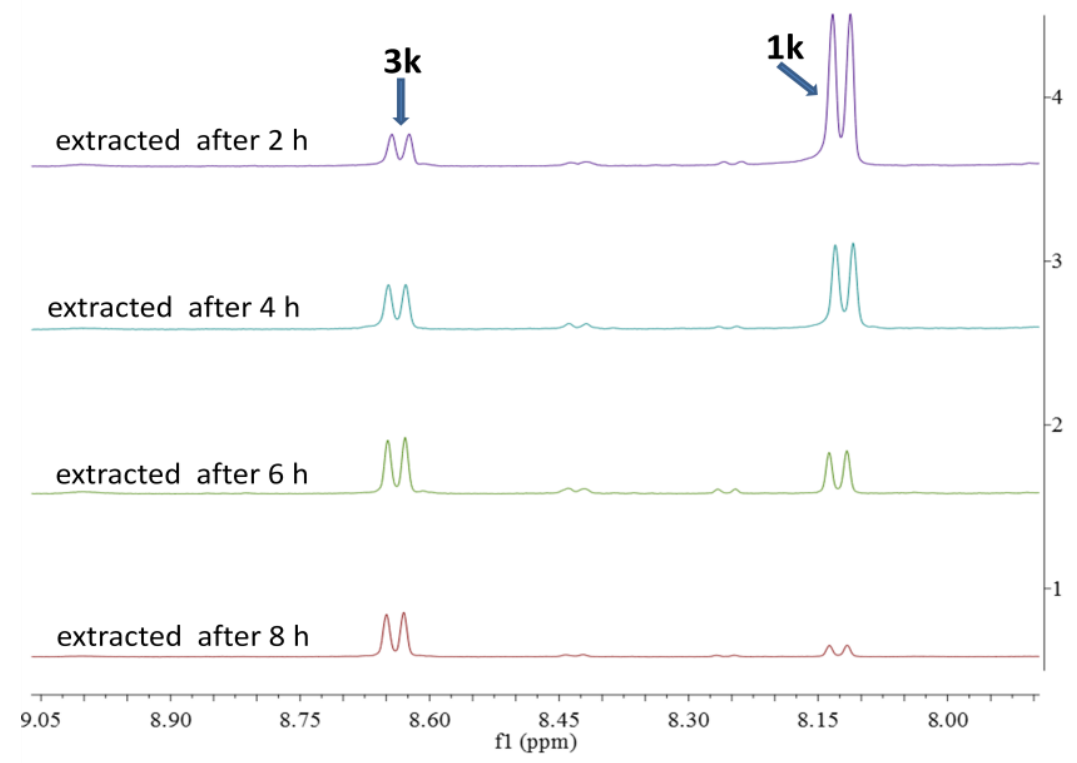

Figure 3, The conversion of $\mathbf{1 k}$ was monitored by ${ }^{1} \mathrm{H}$ NMR method 


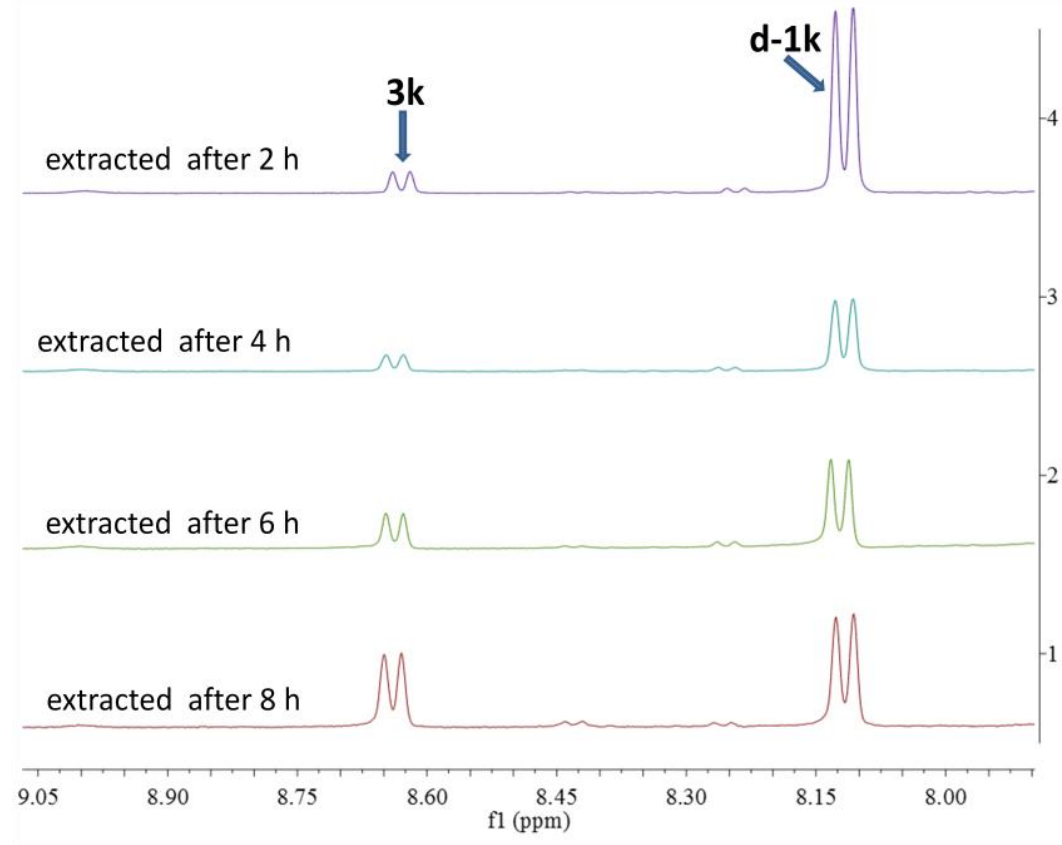

Figure 4. The conversion of $\boldsymbol{d}-\mathbf{1 k}$ was monitored by ${ }^{1} \mathrm{H}$ NMR method

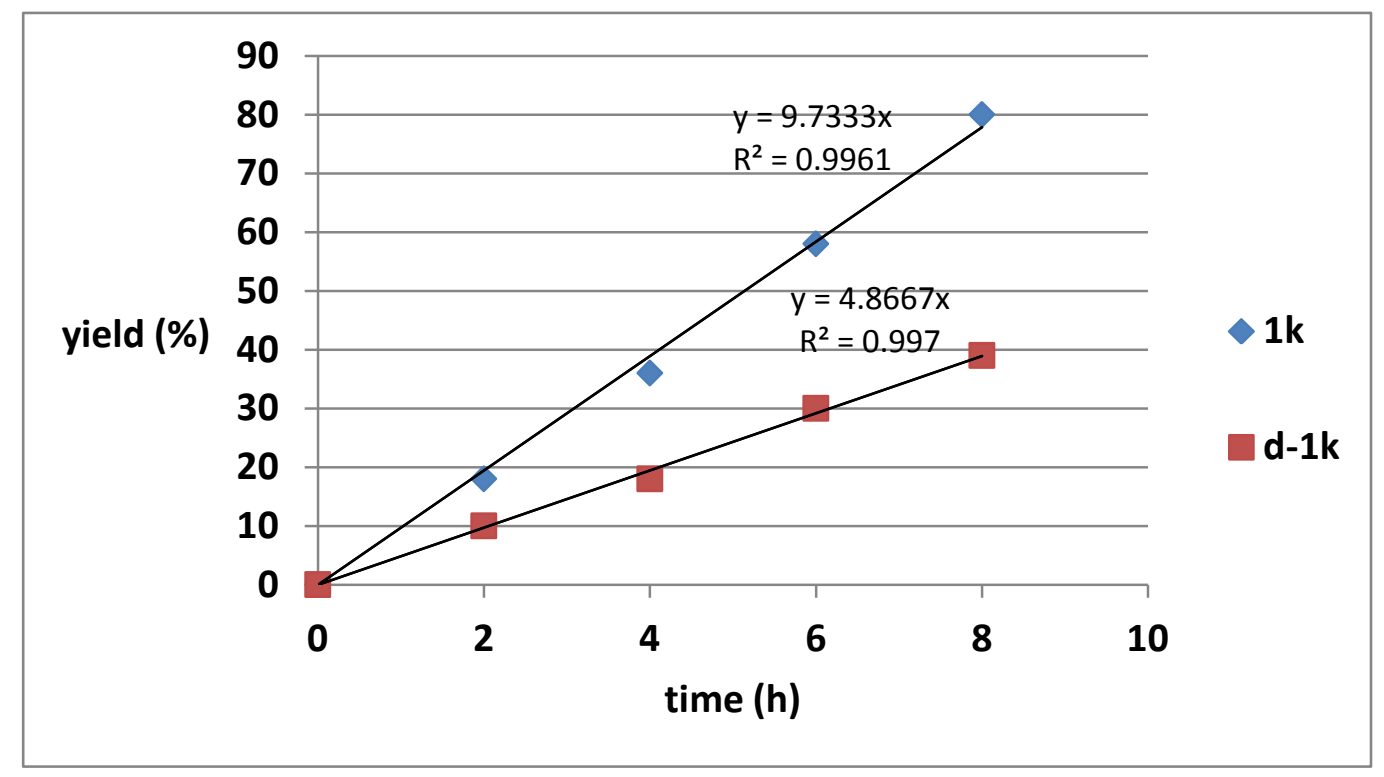

Figure 5. The plot of initial rates for KIE measurements 


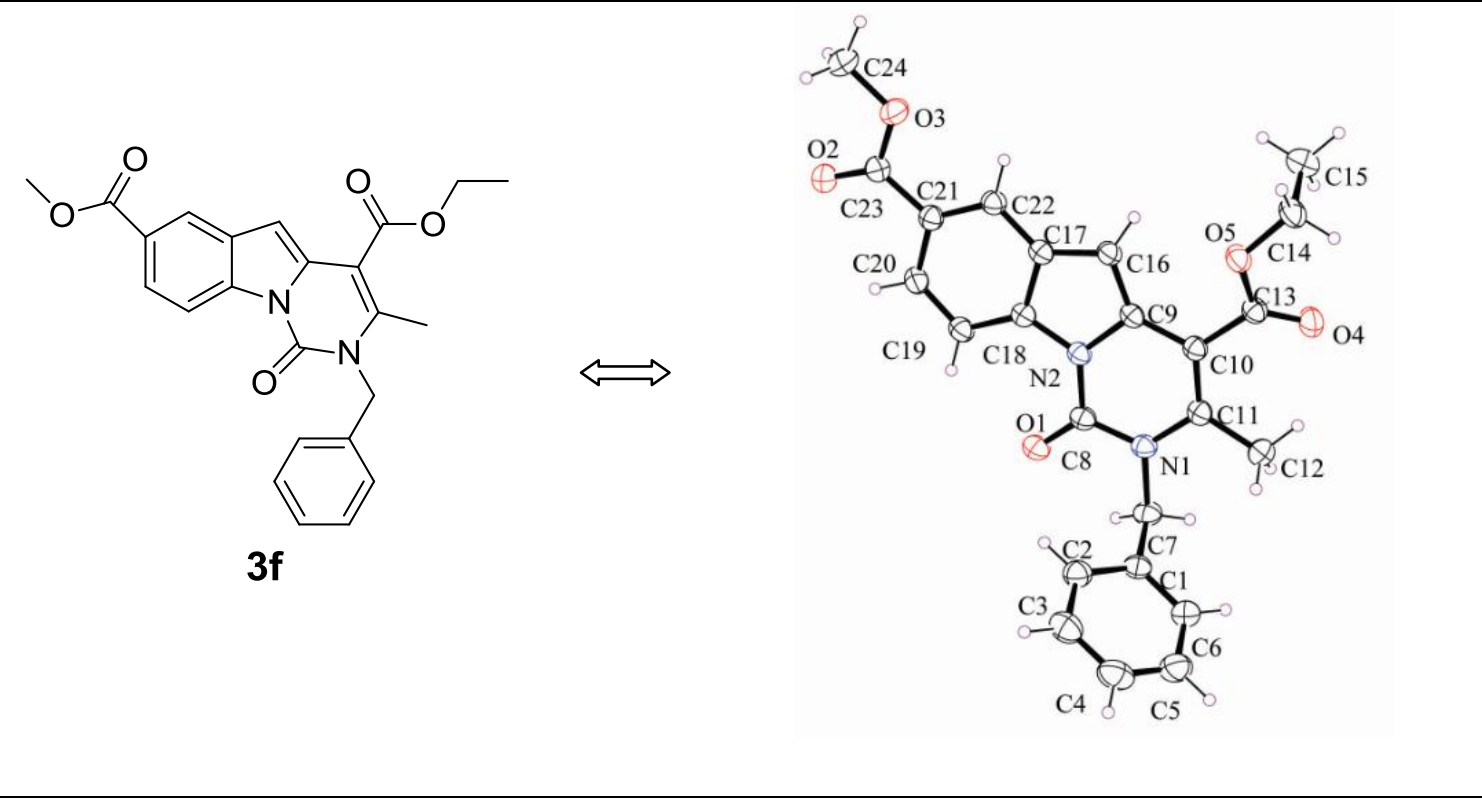

Figure 1. The Single crystal structure of compound $3 f$

Table 7. Crystal data and structure refinement for $3 f$.

Identification code

Empirical formula

Formula weight

Temperature

Wavelength

Crystal system, space group

Unit cell dimensions

Volume

Z, Calculated density

Absorption coefficient

$\mathrm{F}(000)$

Crystal size

Theta range for data collection

Limiting indices

Reflections collected / unique

Completeness to theta $=27.51$

Refinement method

Data / restraints / parameters
$3 \mathrm{f}$

$\mathrm{C}_{24} \mathrm{H}_{22} \mathrm{~N}_{2} \mathrm{O}_{5}$

418.44

296(2) K

$0.71073 \mathrm{~A}$

Monoclinic, $\mathrm{C} 2 / \mathrm{C}$

$\mathrm{a}=8.322(6) \mathrm{A} \quad$ alpha $=90 \mathrm{deg}$.

$\mathrm{b}=21.996(16)$ A $\quad$ beta $=107.208(8)$ deg .

$\mathrm{c}=11.667(9) \mathrm{A} \quad$ gamma $=90 \mathrm{deg}$.

2040(3) $\mathrm{A}^{\wedge} 3$

4, $1.362 \mathrm{Mg} / \mathrm{m}^{\wedge} 3$

$0.096 \mathrm{~mm}^{\wedge}-1$

880

$0.28 \times 0.21 \times 0.20 \mathrm{~mm}^{3}$

1.85 to $27.51 \mathrm{deg}$.

$-10<=\mathrm{h}<=10,-28<=\mathrm{k}<=27,-14<=1<=14$

$22581 / 4569[\mathrm{R}(\mathrm{int})=0.0196]$

$97.2 \%$

Full-matrix least-squares on $\mathrm{F}^{\wedge} 2$

4569 / 0 / 280 
Goodness-of-fit on $\mathrm{F}^{\wedge} 2 \quad 1.050$

Final $\mathrm{R}$ indices $[\mathrm{I}>2 \operatorname{sigma}(\mathrm{I})] \quad \mathrm{R} 1=0.0452, \mathrm{wR} 2=0.1230$

$\mathrm{R}$ indices (all data) $\quad \mathrm{R} 1=0.0613, \mathrm{wR} 2=0.1372$

Largest diff. peak and hole $\quad 0.188$ and -0.202 e.A^^-3

Table 8. Atomic coordinates $\left(\times 10^{4}\right)$ and equivalent isotropic displacement parameters $\left(\AA^{2} \times\right.$ $10^{3}$ ) for $3 \mathbf{f}$.

$\mathrm{U}(\mathrm{eq})$ is defined as one third of the trace of the orthogonalized $\mathrm{U}^{\mathrm{ij}}$ tensor.

\begin{tabular}{|c|c|c|c|c|}
\hline & $\mathrm{X}$ & $\mathrm{y}$ & $\mathrm{z}$ & $\mathrm{U}(\mathrm{eq})$ \\
\hline $\mathrm{O}(1)$ & $-2149(2)$ & $4766(1)$ & $12423(1)$ & $58(1)$ \\
\hline $\mathrm{O}(2)$ & $-6090(2)$ & $7188(1)$ & $8640(1)$ & $73(1)$ \\
\hline $\mathrm{O}(3)$ & $-5211(2)$ & $6885(1)$ & $7108(1)$ & $66(1)$ \\
\hline $\mathrm{O}(4)$ & $-85(2)$ & $3016(1)$ & $8775(1)$ & $65(1)$ \\
\hline $\mathrm{O}(5)$ & $-116(1)$ & $3944(1)$ & 7997(1) & $54(1)$ \\
\hline $\mathrm{N}(1)$ & $-862(2)$ & $3957(1)$ & 11827(1) & $45(1)$ \\
\hline $\mathrm{N}(2)$ & $-2101(1)$ & 4731(1) & $10490(1)$ & $41(1)$ \\
\hline $\mathrm{C}(1)$ & 1071(2) & $3868(1)$ & 13941(1) & $48(1)$ \\
\hline $\mathrm{C}(2)$ & $1453(2)$ & $4470(1)$ & $14284(2)$ & $57(1)$ \\
\hline $\mathrm{C}(3)$ & $2920(2)$ & $4615(1)$ & $15160(2)$ & $71(1)$ \\
\hline $\mathrm{C}(4)$ & $4023(2)$ & $4163(1)$ & $15706(2)$ & $77(1)$ \\
\hline$C(5)$ & $3670(3)$ & $3569(1)$ & $15384(2)$ & $74(1)$ \\
\hline$C(6)$ & 2191(2) & $3421(1)$ & $14501(2)$ & $60(1)$ \\
\hline $\mathrm{C}(7)$ & $-582(2)$ & $3699(1)$ & $13043(1)$ & $50(1)$ \\
\hline $\mathrm{C}(8)$ & $-1738(2)$ & $4504(1)$ & $11637(1)$ & $45(1)$ \\
\hline $\mathrm{C}(9)$ & $-1644(2)$ & $4456(1)$ & $9540(1)$ & $41(1)$ \\
\hline$C(10)$ & $-760(2)$ & $3884(1)$ & $9804(1)$ & $42(1)$ \\
\hline $\mathrm{C}(11)$ & $-388(2)$ & $3647(1)$ & 10931(1) & $44(1)$ \\
\hline$C(12)$ & $575(2)$ & $3071(1)$ & $11328(2)$ & $59(1)$ \\
\hline$C(13)$ & $-286(2)$ & $3558(1)$ & $8836(1)$ & $46(1)$ \\
\hline$C(14)$ & $239(2)$ & $3687(1)$ & $6953(2)$ & $58(1)$ \\
\hline$C(15)$ & $-1344(3)$ & $3512(1)$ & $6031(2)$ & $70(1)$ \\
\hline$C(16)$ & $-2220(2)$ & $4817(1)$ & $8548(1)$ & $44(1)$ \\
\hline $\mathrm{C}(17)$ & $-3058(2)$ & $5326(1)$ & $8869(1)$ & $42(1)$ \\
\hline $\mathrm{C}(18)$ & $-2982(2)$ & $5267(1)$ & $10082(1)$ & $41(1)$ \\
\hline$C(19)$ & $-3719(2)$ & $5687(1)$ & $10666(1)$ & $48(1)$ \\
\hline $\mathrm{C}(20)$ & $-4503(2)$ & $6179(1)$ & 10011(1) & $49(1)$ \\
\hline
\end{tabular}




$\begin{array}{lllll}\mathrm{C}(21) & -4563(2) & 6259(1) & 8808(1) & 45(1) \\ \mathrm{C}(22) & -3853(2) & 5833(1) & 8226(1) & 45(1) \\ \mathrm{C}(23) & -5365(2) & 6821(1) & 8206(1) & 50(1) \\ \mathrm{C}(24) & -5868(3) & 7439(1) & 6491(2) & 79(1)\end{array}$

Table 9. Bond lengths $[\mathrm{A}]$ and angles [deg] for $3 f$.

\begin{tabular}{ll}
\hline $\mathrm{O}(1)-\mathrm{C}(8)$ & $1.2146(18)$ \\
$\mathrm{O}(2)-\mathrm{C}(23)$ & $1.2048(19)$ \\
$\mathrm{O}(3)-\mathrm{C}(23)$ & $1.332(2)$ \\
$\mathrm{O}(3)-\mathrm{C}(24)$ & $1.438(2)$ \\
$\mathrm{O}(4)-\mathrm{C}(13)$ & $1.209(2)$ \\
$\mathrm{O}(5)-\mathrm{C}(13)$ & $1.3339(19)$ \\
$\mathrm{O}(5)-\mathrm{C}(14)$ & $1.4509(19)$ \\
$\mathrm{N}(1)-\mathrm{C}(8)$ & $1.391(2)$ \\
$\mathrm{N}(1)-\mathrm{C}(11)$ & $1.3987(19)$ \\
$\mathrm{N}(1)-\mathrm{C}(7)$ & $1.481(2)$ \\
$\mathrm{N}(2)-\mathrm{C}(8)$ & $1.376(2)$ \\
$\mathrm{N}(2)-\mathrm{C}(18)$ & $1.3955(19)$ \\
$\mathrm{N}(2)-\mathrm{C}(9)$ & $1.4106(18)$ \\
$\mathrm{C}(1)-\mathrm{C}(6)$ & $1.380(2)$ \\
$\mathrm{C}(1)-\mathrm{C}(2)$ & $1.391(2)$ \\
$\mathrm{C}(1)-\mathrm{C}(7)$ & $1.509(2)$ \\
$\mathrm{C}(2)-\mathrm{C}(3)$ & $1.378(3)$ \\
$\mathrm{C}(2)-\mathrm{H}(2 \mathrm{~A})$ & 0.9300 \\
$\mathrm{C}(3)-\mathrm{C}(4)$ & $1.375(3)$ \\
$\mathrm{C}(3)-\mathrm{H}(3 \mathrm{~A})$ & 0.9300 \\
$\mathrm{C}(4)-\mathrm{C}(5)$ & $1.369(3)$ \\
$\mathrm{C}(4)-\mathrm{H}(4 \mathrm{~A})$ & 0.9300 \\
$\mathrm{C}(5)-\mathrm{C}(6)$ & $1.390(3)$ \\
$\mathrm{C}(5)-\mathrm{H}(5 \mathrm{~A})$ & 0.9300 \\
$\mathrm{C}(6)-\mathrm{H}(6 \mathrm{~A})$ & 0.9300 \\
$\mathrm{C}(7)-\mathrm{H}(7 \mathrm{~A})$ & 0.9700 \\
$\mathrm{C}(7)-\mathrm{H}(7 \mathrm{~B})$ & 0.9700 \\
$\mathrm{C}(9)-\mathrm{C}(16)$ & $1.368(2)$ \\
$\mathrm{C}(9)-\mathrm{C}(10)$ & $1.444(2)$ \\
$\mathrm{C}(10)-\mathrm{C}(11)$ & $1.361(2)$ \\
&
\end{tabular}




\begin{tabular}{|c|c|}
\hline $\mathrm{C}(10)-\mathrm{C}(13)$ & $1.486(2)$ \\
\hline $\mathrm{C}(11)-\mathrm{C}(12)$ & $1.498(2)$ \\
\hline $\mathrm{C}(12)-\mathrm{H}(12 \mathrm{~A})$ & 0.9600 \\
\hline $\mathrm{C}(12)-\mathrm{H}(12 \mathrm{~B})$ & 0.9600 \\
\hline $\mathrm{C}(12)-\mathrm{H}(12 \mathrm{C})$ & 0.9600 \\
\hline$C(14)-C(15)$ & $1.484(3)$ \\
\hline $\mathrm{C}(14)-\mathrm{H}(14 \mathrm{~A})$ & 0.9700 \\
\hline $\mathrm{C}(14)-\mathrm{H}(14 \mathrm{~B})$ & 0.9700 \\
\hline $\mathrm{C}(15)-\mathrm{H}(15 \mathrm{~A})$ & 0.9600 \\
\hline $\mathrm{C}(15)-\mathrm{H}(15 \mathrm{~B})$ & 0.9600 \\
\hline $\mathrm{C}(15)-\mathrm{H}(15 \mathrm{C})$ & 0.9600 \\
\hline $\mathrm{C}(16)-\mathrm{C}(17)$ & $1.427(2)$ \\
\hline $\mathrm{C}(16)-\mathrm{H}(16 \mathrm{~A})$ & 0.9300 \\
\hline $\mathrm{C}(17)-\mathrm{C}(22)$ & $1.397(2)$ \\
\hline $\mathrm{C}(17)-\mathrm{C}(18)$ & $1.404(2)$ \\
\hline $\mathrm{C}(18)-\mathrm{C}(19)$ & $1.392(2)$ \\
\hline $\mathrm{C}(19)-\mathrm{C}(20)$ & $1.374(2)$ \\
\hline $\mathrm{C}(19)-\mathrm{H}(19 \mathrm{~A})$ & 0.9300 \\
\hline$C(20)-C(21)$ & $1.402(2)$ \\
\hline $\mathrm{C}(20)-\mathrm{H}(20 \mathrm{~A})$ & 0.9300 \\
\hline$C(21)-C(22)$ & $1.387(2)$ \\
\hline$C(21)-C(23)$ & $1.480(2)$ \\
\hline $\mathrm{C}(22)-\mathrm{H}(22 \mathrm{~A})$ & 0.9300 \\
\hline $\mathrm{C}(24)-\mathrm{H}(24 \mathrm{~A})$ & 0.9600 \\
\hline $\mathrm{C}(24)-\mathrm{H}(24 \mathrm{~B})$ & 0.9600 \\
\hline $\mathrm{C}(24)-\mathrm{H}(24 \mathrm{C})$ & 0.9600 \\
\hline $\mathrm{C}(23)-\mathrm{O}(3)-\mathrm{C}(24)$ & $116.10(14)$ \\
\hline $\mathrm{C}(13)-\mathrm{O}(5)-\mathrm{C}(14)$ & $117.33(13)$ \\
\hline $\mathrm{C}(8)-\mathrm{N}(1)-\mathrm{C}(11)$ & $123.59(12)$ \\
\hline $\mathrm{C}(8)-\mathrm{N}(1)-\mathrm{C}(7)$ & $114.23(12)$ \\
\hline $\mathrm{C}(11)-\mathrm{N}(1)-\mathrm{C}(7)$ & $122.01(13)$ \\
\hline $\mathrm{C}(8)-\mathrm{N}(2)-\mathrm{C}(18)$ & $125.46(12)$ \\
\hline $\mathrm{C}(8)-\mathrm{N}(2)-\mathrm{C}(9)$ & $125.12(13)$ \\
\hline $\mathrm{C}(18)-\mathrm{N}(2)-\mathrm{C}(9)$ & $109.42(11)$ \\
\hline$C(6)-C(1)-C(2)$ & $118.53(16)$ \\
\hline$C(6)-C(1)-C(7)$ & $120.11(15)$ \\
\hline$C(2)-C(1)-C(7)$ & $121.22(14)$ \\
\hline
\end{tabular}




\begin{tabular}{|c|c|}
\hline $\mathrm{C}(3)-\mathrm{C}(2)-\mathrm{C}(1)$ & $120.71(17)$ \\
\hline $\mathrm{C}(3)-\mathrm{C}(2)-\mathrm{H}(2 \mathrm{~A})$ & 119.6 \\
\hline $\mathrm{C}(1)-\mathrm{C}(2)-\mathrm{H}(2 \mathrm{~A})$ & 119.6 \\
\hline $\mathrm{C}(4)-\mathrm{C}(3)-\mathrm{C}(2)$ & $119.99(19)$ \\
\hline $\mathrm{C}(4)-\mathrm{C}(3)-\mathrm{H}(3 \mathrm{~A})$ & 120.0 \\
\hline $\mathrm{C}(2)-\mathrm{C}(3)-\mathrm{H}(3 \mathrm{~A})$ & 120.0 \\
\hline$C(5)-C(4)-C(3)$ & $120.23(19)$ \\
\hline $\mathrm{C}(5)-\mathrm{C}(4)-\mathrm{H}(4 \mathrm{~A})$ & 119.9 \\
\hline $\mathrm{C}(3)-\mathrm{C}(4)-\mathrm{H}(4 \mathrm{~A})$ & 119.9 \\
\hline$C(4)-C(5)-C(6)$ & $119.89(19)$ \\
\hline $\mathrm{C}(4)-\mathrm{C}(5)-\mathrm{H}(5 \mathrm{~A})$ & 120.1 \\
\hline $\mathrm{C}(6)-\mathrm{C}(5)-\mathrm{H}(5 \mathrm{~A})$ & 120.1 \\
\hline$C(1)-C(6)-C(5)$ & $120.65(18)$ \\
\hline $\mathrm{C}(1)-\mathrm{C}(6)-\mathrm{H}(6 \mathrm{~A})$ & 119.7 \\
\hline $\mathrm{C}(5)-\mathrm{C}(6)-\mathrm{H}(6 \mathrm{~A})$ & 119.7 \\
\hline $\mathrm{N}(1)-\mathrm{C}(7)-\mathrm{C}(1)$ & $115.08(12)$ \\
\hline $\mathrm{N}(1)-\mathrm{C}(7)-\mathrm{H}(7 \mathrm{~A})$ & 108.5 \\
\hline $\mathrm{C}(1)-\mathrm{C}(7)-\mathrm{H}(7 \mathrm{~A})$ & 108.5 \\
\hline $\mathrm{N}(1)-\mathrm{C}(7)-\mathrm{H}(7 \mathrm{~B})$ & 108.5 \\
\hline $\mathrm{C}(1)-\mathrm{C}(7)-\mathrm{H}(7 \mathrm{~B})$ & 108.5 \\
\hline $\mathrm{H}(7 \mathrm{~A})-\mathrm{C}(7)-\mathrm{H}(7 \mathrm{~B})$ & 107.5 \\
\hline $\mathrm{O}(1)-\mathrm{C}(8)-\mathrm{N}(2)$ & $122.14(14)$ \\
\hline $\mathrm{O}(1)-\mathrm{C}(8)-\mathrm{N}(1)$ & $122.83(14)$ \\
\hline $\mathrm{N}(2)-\mathrm{C}(8)-\mathrm{N}(1)$ & $115.03(12)$ \\
\hline $\mathrm{C}(16)-\mathrm{C}(9)-\mathrm{N}(2)$ & $107.84(13)$ \\
\hline$C(16)-C(9)-C(10)$ & $135.68(13)$ \\
\hline $\mathrm{N}(2)-\mathrm{C}(9)-\mathrm{C}(10)$ & $116.47(12)$ \\
\hline$C(11)-C(10)-C(9)$ & $119.71(13)$ \\
\hline$C(11)-C(10)-C(13)$ & $120.95(14)$ \\
\hline$C(9)-C(10)-C(13)$ & $119.34(13)$ \\
\hline $\mathrm{C}(10)-\mathrm{C}(11)-\mathrm{N}(1)$ & $120.06(14)$ \\
\hline $\mathrm{C}(10)-\mathrm{C}(11)-\mathrm{C}(12)$ & $124.77(14)$ \\
\hline $\mathrm{N}(1)-\mathrm{C}(11)-\mathrm{C}(12)$ & $115.13(13)$ \\
\hline $\mathrm{C}(11)-\mathrm{C}(12)-\mathrm{H}(12 \mathrm{~A})$ & 109.5 \\
\hline $\mathrm{C}(11)-\mathrm{C}(12)-\mathrm{H}(12 \mathrm{~B})$ & 109.5 \\
\hline $\mathrm{H}(12 \mathrm{~A})-\mathrm{C}(12)-\mathrm{H}(12 \mathrm{~B})$ & 109.5 \\
\hline $\mathrm{C}(11)-\mathrm{C}(12)-\mathrm{H}(12 \mathrm{C})$ & 109.5 \\
\hline $\mathrm{H}(12 \mathrm{~A})-\mathrm{C}(12)-\mathrm{H}(12 \mathrm{C})$ & 109.5 \\
\hline
\end{tabular}




\begin{tabular}{|c|c|}
\hline $\mathrm{H}(12 \mathrm{~B})-\mathrm{C}(12)-\mathrm{H}(12 \mathrm{C})$ & 109.5 \\
\hline $\mathrm{O}(4)-\mathrm{C}(13)-\mathrm{O}(5)$ & $122.58(14)$ \\
\hline $\mathrm{O}(4)-\mathrm{C}(13)-\mathrm{C}(10)$ & $126.26(14)$ \\
\hline $\mathrm{O}(5)-\mathrm{C}(13)-\mathrm{C}(10)$ & $111.15(13)$ \\
\hline $\mathrm{O}(5)-\mathrm{C}(14)-\mathrm{C}(15)$ & $110.64(15)$ \\
\hline $\mathrm{O}(5)-\mathrm{C}(14)-\mathrm{H}(14 \mathrm{~A})$ & 109.5 \\
\hline $\mathrm{C}(15)-\mathrm{C}(14)-\mathrm{H}(14 \mathrm{~A})$ & 109.5 \\
\hline $\mathrm{O}(5)-\mathrm{C}(14)-\mathrm{H}(14 \mathrm{~B})$ & 109.5 \\
\hline $\mathrm{C}(15)-\mathrm{C}(14)-\mathrm{H}(14 \mathrm{~B})$ & 109.5 \\
\hline $\mathrm{H}(14 \mathrm{~A})-\mathrm{C}(14)-\mathrm{H}(14 \mathrm{~B})$ & 108.1 \\
\hline $\mathrm{C}(14)-\mathrm{C}(15)-\mathrm{H}(15 \mathrm{~A})$ & 109.5 \\
\hline $\mathrm{C}(14)-\mathrm{C}(15)-\mathrm{H}(15 \mathrm{~B})$ & 109.5 \\
\hline $\mathrm{H}(15 \mathrm{~A})-\mathrm{C}(15)-\mathrm{H}(15 \mathrm{~B})$ & 109.5 \\
\hline $\mathrm{C}(14)-\mathrm{C}(15)-\mathrm{H}(15 \mathrm{C})$ & 109.5 \\
\hline $\mathrm{H}(15 \mathrm{~A})-\mathrm{C}(15)-\mathrm{H}(15 \mathrm{C})$ & 109.5 \\
\hline $\mathrm{H}(15 \mathrm{~B})-\mathrm{C}(15)-\mathrm{H}(15 \mathrm{C})$ & 109.5 \\
\hline $\mathrm{C}(9)-\mathrm{C}(16)-\mathrm{C}(17)$ & $108.09(13)$ \\
\hline $\mathrm{C}(9)-\mathrm{C}(16)-\mathrm{H}(16 \mathrm{~A})$ & 126.0 \\
\hline $\mathrm{C}(17)-\mathrm{C}(16)-\mathrm{H}(16 \mathrm{~A})$ & 126.0 \\
\hline $\mathrm{C}(22)-\mathrm{C}(17)-\mathrm{C}(18)$ & $119.18(13)$ \\
\hline$C(22)-C(17)-C(16)$ & $132.64(14)$ \\
\hline$C(18)-C(17)-C(16)$ & $108.18(13)$ \\
\hline $\mathrm{C}(19)-\mathrm{C}(18)-\mathrm{N}(2)$ & $131.00(13)$ \\
\hline$C(19)-C(18)-C(17)$ & $122.52(14)$ \\
\hline $\mathrm{N}(2)-\mathrm{C}(18)-\mathrm{C}(17)$ & $106.47(12)$ \\
\hline$C(20)-C(19)-C(18)$ & $117.08(14)$ \\
\hline $\mathrm{C}(20)-\mathrm{C}(19)-\mathrm{H}(19 \mathrm{~A})$ & 121.5 \\
\hline C(18)-C(19)-H(19A) & 121.5 \\
\hline$C(19)-C(20)-C(21)$ & $121.80(14)$ \\
\hline $\mathrm{C}(19)-\mathrm{C}(20)-\mathrm{H}(20 \mathrm{~A})$ & 119.1 \\
\hline $\mathrm{C}(21)-\mathrm{C}(20)-\mathrm{H}(20 \mathrm{~A})$ & 119.1 \\
\hline $\mathrm{C}(22)-\mathrm{C}(21)-\mathrm{C}(20)$ & $120.71(14)$ \\
\hline $\mathrm{C}(22)-\mathrm{C}(21)-\mathrm{C}(23)$ & $122.04(14)$ \\
\hline$C(20)-C(21)-C(23)$ & $117.22(13)$ \\
\hline $\mathrm{C}(21)-\mathrm{C}(22)-\mathrm{C}(17)$ & $118.67(14)$ \\
\hline $\mathrm{C}(21)-\mathrm{C}(22)-\mathrm{H}(22 \mathrm{~A})$ & 120.7 \\
\hline $\mathrm{C}(17)-\mathrm{C}(22)-\mathrm{H}(22 \mathrm{~A})$ & 120.7 \\
\hline $\mathrm{O}(2)-\mathrm{C}(23)-\mathrm{O}(3)$ & $122.40(15)$ \\
\hline
\end{tabular}




$\begin{array}{ll}\mathrm{O}(2)-\mathrm{C}(23)-\mathrm{C}(21) & 124.95(15) \\ \mathrm{O}(3)-\mathrm{C}(23)-\mathrm{C}(21) & 112.65(13) \\ \mathrm{O}(3)-\mathrm{C}(24)-\mathrm{H}(24 \mathrm{~A}) & 109.5 \\ \mathrm{O}(3)-\mathrm{C}(24)-\mathrm{H}(24 \mathrm{~B}) & 109.5 \\ \mathrm{H}(24 \mathrm{~A})-\mathrm{C}(24)-\mathrm{H}(24 \mathrm{~B}) & 109.5 \\ \mathrm{O}(3)-\mathrm{C}(24)-\mathrm{H}(24 \mathrm{C}) & 109.5 \\ \mathrm{H}(24 \mathrm{~A})-\mathrm{C}(24)-\mathrm{H}(24 \mathrm{C}) & 109.5 \\ \mathrm{H}(24 \mathrm{~B})-\mathrm{C}(24)-\mathrm{H}(24 \mathrm{C}) & 109.5\end{array}$

Symmetry transformations used to generate equivalent atoms:

Table 10. Anisotropic displacement parameters $\left(A^{\wedge} 2 \times 10^{\wedge} 3\right)$ for 3f. The anisotropic displacement factor exponent takes the form: $-2 \pi^{2}\left[\mathrm{~h}^{2} \mathrm{a}^{2} \mathrm{U}^{11}+\ldots+2 \mathrm{hka} * \mathrm{~b} * \mathrm{U}^{12}\right]$

\begin{tabular}{lcccccc}
\hline & $\mathrm{U} 11$ & $\mathrm{U} 22$ & $\mathrm{U} 33$ & $\mathrm{U} 23$ & $\mathrm{U} 13$ & $\mathrm{U} 12$ \\
\hline $\mathrm{O}(1)$ & $70(1)$ & $67(1)$ & $42(1)$ & $2(1)$ & $23(1)$ & $9(1)$ \\
$\mathrm{O}(2)$ & $102(1)$ & $58(1)$ & $60(1)$ & $1(1)$ & $27(1)$ & $25(1)$ \\
$\mathrm{O}(3)$ & $89(1)$ & $59(1)$ & $56(1)$ & $14(1)$ & $31(1)$ & $19(1)$ \\
$\mathrm{O}(4)$ & $97(1)$ & $43(1)$ & $61(1)$ & $-4(1)$ & $31(1)$ & $4(1)$ \\
$\mathrm{O}(5)$ & $71(1)$ & $48(1)$ & $50(1)$ & $-4(1)$ & $30(1)$ & $-1(1)$ \\
$\mathrm{N}(1)$ & $51(1)$ & $45(1)$ & $39(1)$ & $3(1)$ & $14(1)$ & $-5(1)$ \\
$\mathrm{N}(2)$ & $44(1)$ & $41(1)$ & $37(1)$ & $-2(1)$ & $14(1)$ & $-4(1)$ \\
$\mathrm{C}(1)$ & $53(1)$ & $54(1)$ & $39(1)$ & $5(1)$ & $17(1)$ & $-2(1)$ \\
$\mathrm{C}(2)$ & $64(1)$ & $56(1)$ & $51(1)$ & $2(1)$ & $15(1)$ & $-3(1)$ \\
$\mathrm{C}(3)$ & $72(1)$ & $74(1)$ & $64(1)$ & $-12(1)$ & $16(1)$ & $-16(1)$ \\
$\mathrm{C}(4)$ & $58(1)$ & $107(2)$ & $59(1)$ & $-11(1)$ & $8(1)$ & $-4(1)$ \\
$\mathrm{C}(5)$ & $65(1)$ & $90(2)$ & $60(1)$ & $4(1)$ & $10(1)$ & $17(1)$ \\
$\mathrm{C}(6)$ & $68(1)$ & $61(1)$ & $50(1)$ & $3(1)$ & $15(1)$ & $7(1)$ \\
$\mathrm{C}(7)$ & $57(1)$ & $52(1)$ & $43(1)$ & $8(1)$ & $17(1)$ & $-6(1)$ \\
$\mathrm{C}(8)$ & $46(1)$ & $49(1)$ & $40(1)$ & $0(1)$ & $14(1)$ & $-5(1)$ \\
$\mathrm{C}(9)$ & $43(1)$ & $42(1)$ & $39(1)$ & $-5(1)$ & $15(1)$ & $-8(1)$ \\
$\mathrm{C}(10)$ & $44(1)$ & $40(1)$ & $41(1)$ & $-3(1)$ & $13(1)$ & $-6(1)$ \\
$\mathrm{C}(11)$ & $46(1)$ & $40(1)$ & $46(1)$ & $-2(1)$ & $14(1)$ & $-8(1)$ \\
$\mathrm{C}(12)$ & $75(1)$ & $47(1)$ & $54(1)$ & $4(1)$ & $18(1)$ & $5(1)$ \\
$\mathrm{C}(13)$ & $48(1)$ & $44(1)$ & $46(1)$ & $-4(1)$ & $14(1)$ & $-4(1)$ \\
$\mathrm{C}(14)$ & $71(1)$ & $58(1)$ & $54(1)$ & $-6(1)$ & $34(1)$ & $0(1)$ \\
$\mathrm{C}(15)$ & $77(1)$ & $82(1)$ & $51(1)$ & $-3(1)$ & $20(1)$ & $-2(1)$ \\
& & & & & & \\
\hline
\end{tabular}




$\begin{array}{lllllll}\mathrm{C}(16) & 51(1) & 45(1) & 38(1) & -2(1) & 16(1) & -1(1) \\ \mathrm{C}(17) & 43(1) & 44(1) & 40(1) & -3(1) & 14(1) & -6(1) \\ \mathrm{C}(18) & 41(1) & 41(1) & 40(1) & -2(1) & 13(1) & -6(1) \\ \mathrm{C}(19) & 54(1) & 50(1) & 41(1) & -4(1) & 18(1) & -2(1) \\ \mathrm{C}(20) & 54(1) & 46(1) & 49(1) & -6(1) & 19(1) & 0(1) \\ \mathrm{C}(21) & 46(1) & 43(1) & 46(1) & -2(1) & 13(1) & -4(1) \\ \mathrm{C}(22) & 50(1) & 45(1) & 41(1) & 1(1) & 15(1) & -3(1) \\ \mathrm{C}(23) & 55(1) & 46(1) & 48(1) & -2(1) & 14(1) & -1(1) \\ \mathrm{C}(24) & 116(2) & 60(1) & 64(1) & 19(1) & 28(1) & 17(1)\end{array}$

Table 11. Hydrogen coordinates $\left(x 1^{\wedge} 4\right)$ and isotropic displacement parameters $\left(A^{\wedge} 2 \times 10^{\wedge} 3\right)$ for 3f.

\begin{tabular}{lcccc}
\hline & $\mathrm{x}$ & $\mathrm{y}$ & $\mathrm{z}$ & $\mathrm{U}(\mathrm{eq})$ \\
\hline $\mathrm{H}(2 \mathrm{~A})$ & 710 & 4777 & 13918 & 69 \\
$\mathrm{H}(3 \mathrm{~A})$ & 3163 & 5019 & 15382 & 85 \\
$\mathrm{H}(4 \mathrm{~A})$ & 5013 & 4262 & 16297 & 92 \\
$\mathrm{H}(5 \mathrm{~A})$ & 4419 & 3264 & 15754 & 88 \\
$\mathrm{H}(6 \mathrm{~A})$ & 1953 & 3016 & 14286 & 72 \\
$\mathrm{H}(7 \mathrm{~A})$ & -644 & 3259 & 12978 & 60 \\
$\mathrm{H}(7 \mathrm{~B})$ & -1487 & 3832 & 13349 & 60 \\
$\mathrm{H}(12 \mathrm{~A})$ & 849 & 2890 & 10660 & 88 \\
$\mathrm{H}(12 \mathrm{~B})$ & -98 & 2793 & 11624 & 88 \\
$\mathrm{H}(12 \mathrm{C})$ & 1592 & 3160 & 11953 & 88 \\
$\mathrm{H}(14 \mathrm{~A})$ & 950 & 3331 & 7190 & 69 \\
$\mathrm{H}(14 \mathrm{~B})$ & 839 & 3982 & 6618 & 69 \\
$\mathrm{H}(15 \mathrm{~A})$ & -1085 & 3343 & 5347 & 105 \\
$\mathrm{H}(15 \mathrm{~B})$ & -2042 & 3865 & 5789 & 105 \\
$\mathrm{H}(15 \mathrm{C})$ & -1929 & 3215 & 6359 & 105 \\
$\mathrm{H}(16 \mathrm{~A})$ & -2089 & 4743 & 7796 & 53 \\
$\mathrm{H}(19 \mathrm{~A})$ & -3681 & 5636 & 11465 & 57 \\
$\mathrm{H}(20 \mathrm{~A})$ & -5009 & 6467 & 10376 & 58 \\
$\mathrm{H}(22 \mathrm{~A})$ & -3906 & 5884 & 7424 & 54 \\
$\mathrm{H}(24 \mathrm{~A})$ & -5697 & 7439 & 5713 & 119 \\
$\mathrm{H}(24 \mathrm{~B})$ & -5296 & 7780 & 6945 & 119 \\
$\mathrm{H}(24 \mathrm{C})$ & -7049 & 7467 & 6404 & 119 \\
\hline & & & &
\end{tabular}




\section{References}

1. (a) Zhang, W.; Wei, J.; Fu, S.; Lin, D.; Jiang, H.; Zeng, W. Org. Lett. 2015, 17, 1349; (b) Sharma, S.; Han, S.; Kim, M.; Mishra, N. K.; Park, J.; Shin, Y.; Ha, J.; Kwak, J. H.; Jung, Y. H.; Kim, I. S. Org. Biomol. Chem. 2014, 12, 1703; (c) Ozuduru, G.; Schubach, T.; Boysen, M. M. K. Org. Lett. 2012, 14, 4990.

2. Chen, X.; Xie, Y.; Xiao, X.; Li, G.; Deng, Y.; Jiang, H.; Zeng, W. Chem. Commun. 2015, 51, 15328.

3. Cernova, M.; Cerna, I.; Pohl, R.; Hocek, M. J. Org. Chem. 2011, 76, 5309.

4. Hartz, R.; Nanda, K.; Ingalls, C.; Ahuja, V.; Molski, T.; Zhang, G.; Wong, H.; Peng, Y.; Kelley, M.; Lodge, N.; Zaczek, R.; Gilligan, P.; Trainor, G. J. Med. Chem. 2004, 47, 4741.

5. Goosen, L. J.; Linder, C.; Rodrguez, N.; Lange, P. P.; Fromm, A. Chem. Commun. 2009, 7173.

6. Cheng, Y.; Bolm, C. Angew. Chem. Int. Ed. 2015, 54, 12349. 
5. ${ }^{1} \mathrm{H}$ NMR and ${ }^{13} \mathrm{C}$ NMR spectrum for all isolated products.

(1) The ${ }^{1} \mathrm{H}$ NMR and ${ }^{13} \mathrm{C}$ NMR spectrum for $\mathbf{3 a}$ (using $\mathrm{CDCl}_{3}$ as solvent)

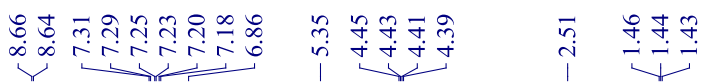<smiles>CCOC(=O)c1c(C)n(Cc2ccccc2)c(=O)n2c1cc1ccccc12</smiles>

$3 a$

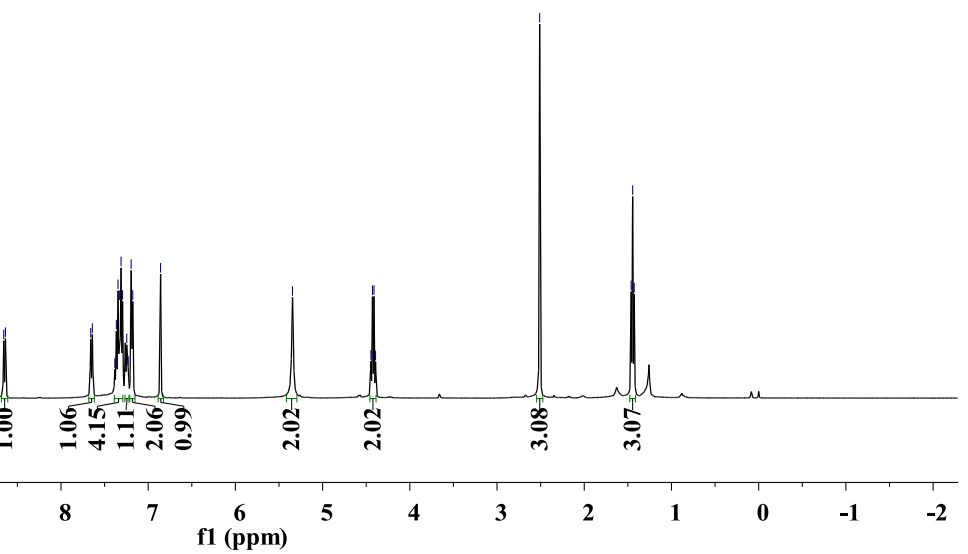

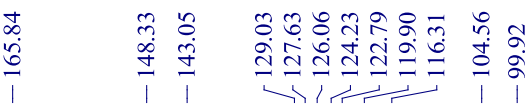

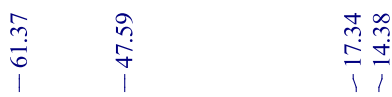<smiles>CCOC(=O)c1c(C)n(Cc2ccccc2)c(=O)n2c1cc1ccccc12</smiles>

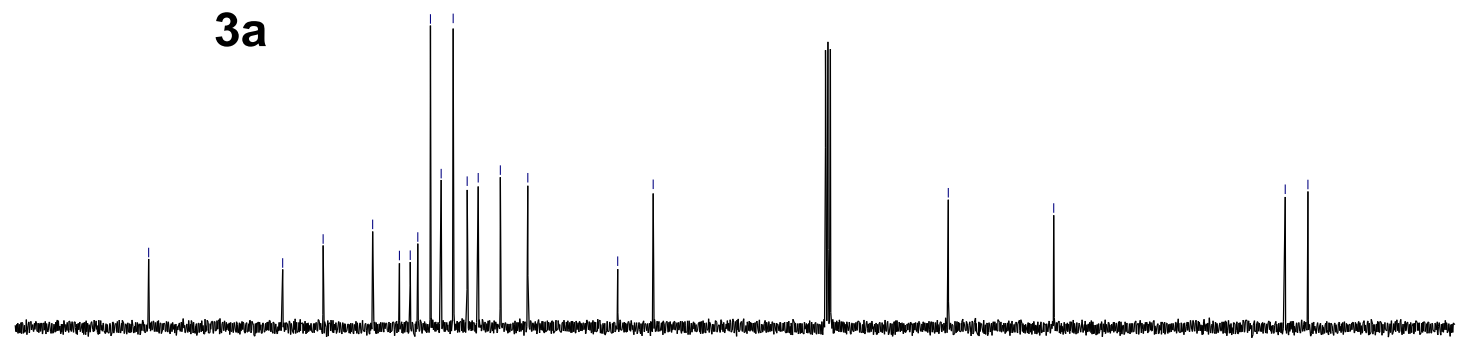

$\begin{array}{lllllllllllllllllll}180 & 170 & 160 & 150 & 140 & 130 & 120 & 110 & 100 \underset{f 1}{90}(\mathrm{ppm}) & 80 & 70 & 60 & 50 & 40 & 30 & 20 & 10 & 0\end{array}$ 
(2) The ${ }^{1} \mathrm{H}$ NMR and ${ }^{13} \mathrm{C}$ NMR spectrum for $\mathbf{3 b}$ (using $\mathrm{CDCl}_{3}$ as solvent)

$$
\text { لro }
$$<smiles></smiles>

$3 b$

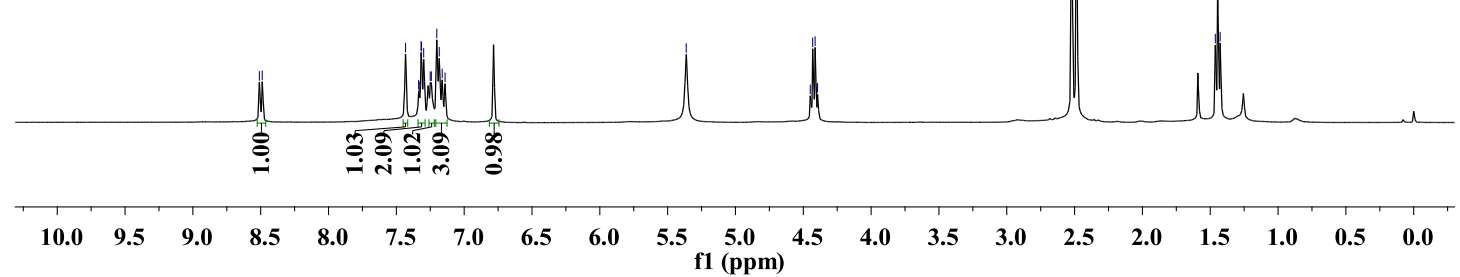

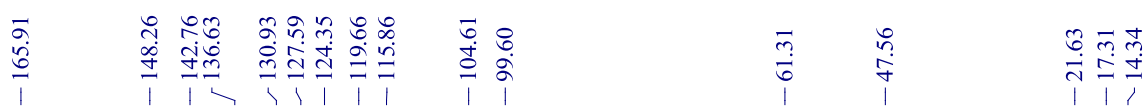<smiles></smiles>

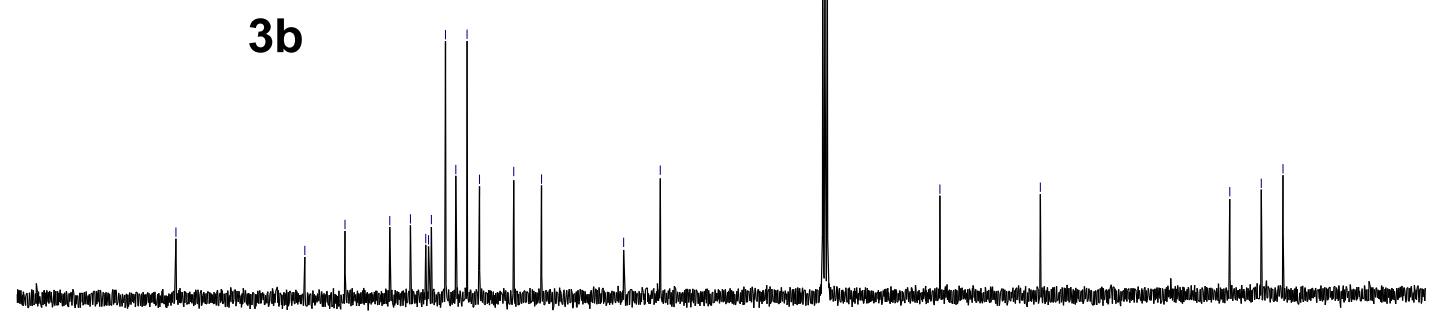

$\begin{array}{lllllllllllllllllll}180 & 170 & 160 & 150 & 140 & 130 & 120 & 110 & 100 & 90 & 80 & 70 & 60 & 50 & 40 & 30 & 20 & 10 & 0\end{array}$ 
(3) The ${ }^{1} \mathrm{H}$ NMR and ${ }^{13} \mathrm{C}$ NMR spectrum for $3 \mathbf{c}$ (using $\mathrm{CDCl}_{3}$ as solvent)

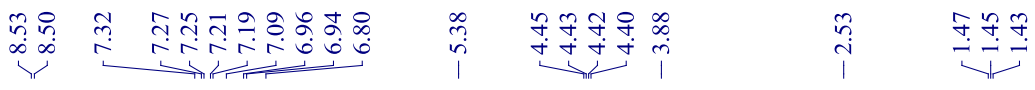<smiles>CCOC(=O)c1c(C)n(Cc2ccccc2)c(=O)n2c1cc1cc(OC)ccc12</smiles>

\section{3c}

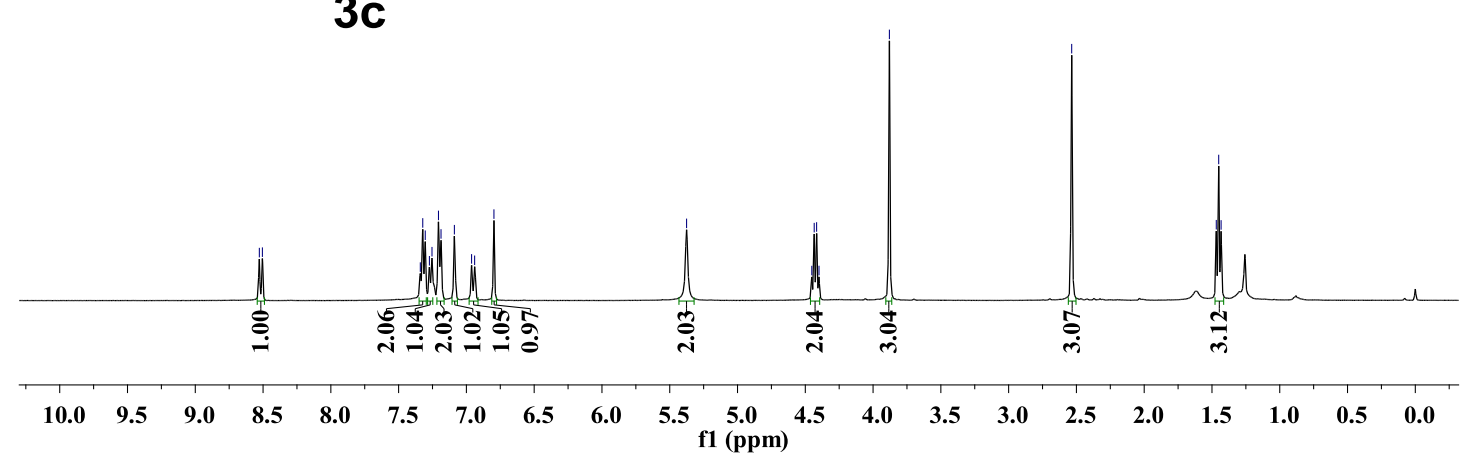

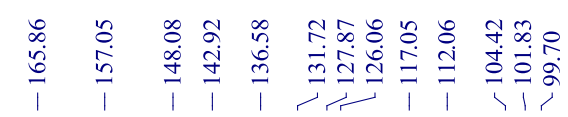

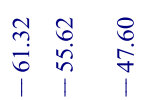

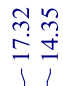<smiles></smiles>

3c

180

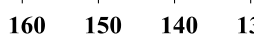

12011

$100 \underset{\text { f1 }}{90} 80$

7060

$\begin{array}{llllll}50 & 40 & 30 & 20 & 10 & 0\end{array}$ 
(4) The ${ }^{1} \mathrm{H}$ NMR and ${ }^{13} \mathrm{C}$ NMR spectrum for $\mathbf{3 d}$ (using $\mathrm{CDCl}_{3}$ as solvent)

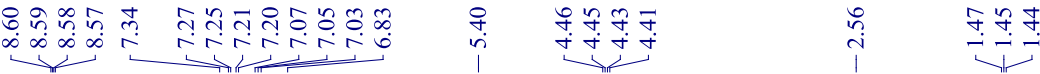<smiles></smiles>

3d

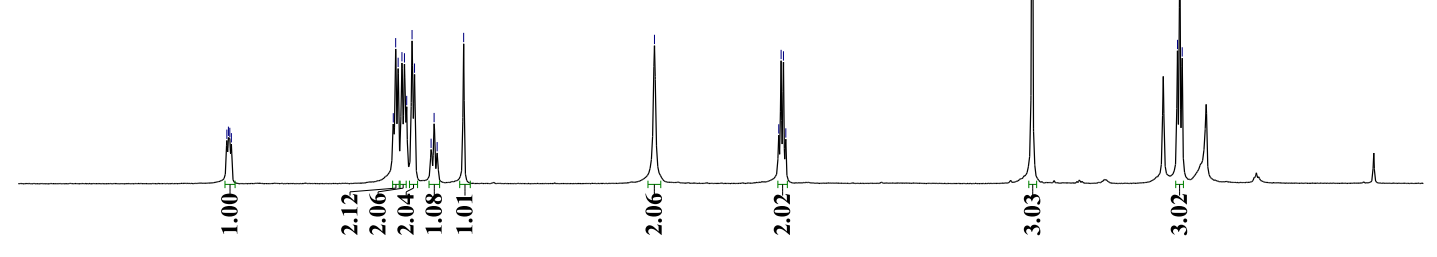

$\begin{array}{lllllllllllllllllllll}10.0 & 9.5 & 9.0 & 8.5 & 8.0 & 7.5 & 7.0 & 6.5 & 6.0 & 5.5 & \begin{array}{c}5.0 \\ \text { f1 (ppm) }\end{array} & 4.5 & 4.0 & 3.5 & 3.0 & 2.5 & 2.0 & 1.5 & 1.0 & 0.5 & 0.0\end{array}$

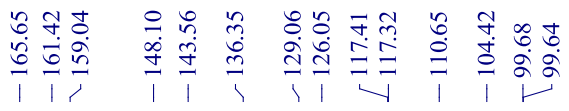

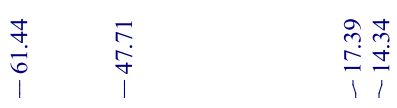<smiles></smiles>

3d

$\begin{array}{lllllllll}170 & 160 & 150 & 140 & 130 & 120 & 110 & 100 & \begin{array}{c}90 \\ \text { f1 (ppm) }\end{array}\end{array}$

$\begin{array}{llllllll}70 & 60 & 50 & 40 & 30 & 20 & 10 & 0\end{array}$ 
(5) The ${ }^{1} \mathrm{H}$ NMR and ${ }^{13} \mathrm{C}$ NMR spectrum for $3 \mathbf{e}$ (using $\mathrm{CDCl}_{3}$ as solvent)

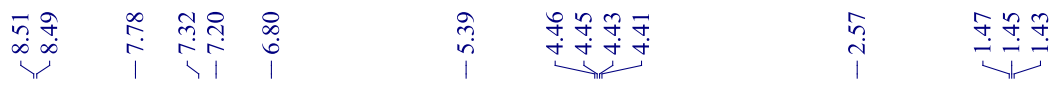<smiles>CCOC(=O)C1=C(C)N(Cc2ccccc2)C(=O)N2C=CC(Br)=CC=C12</smiles>

$3 e$

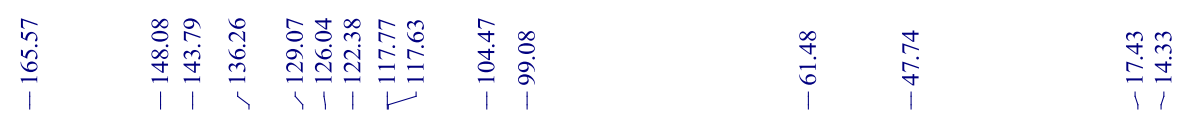<smiles>CCOC(=O)c1c(C)n(Cc2ccccc2)c(=O)n2c1cc1cc(Br)ccc12</smiles>

$3 e$

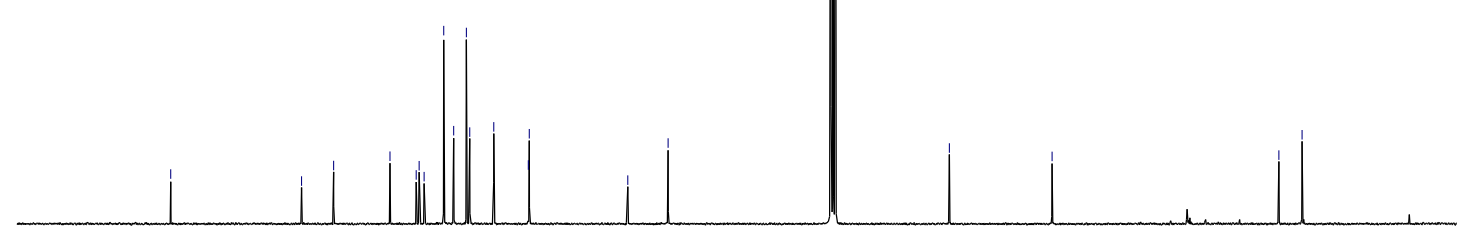

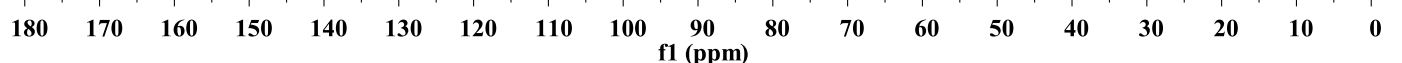


(6) The ${ }^{1} \mathrm{H}$ NMR and ${ }^{13} \mathrm{C}$ NMR spectrum for $\mathbf{3 f}$ (using $\mathrm{CDCl}_{3}$ as solvent)

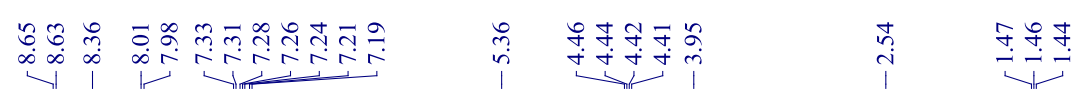<smiles>CCOC(=O)C1=C(C)N(Cc2ccccc2)C(=O)N2C=CC(C(C)=O)=CC=C12</smiles>

$3 f$

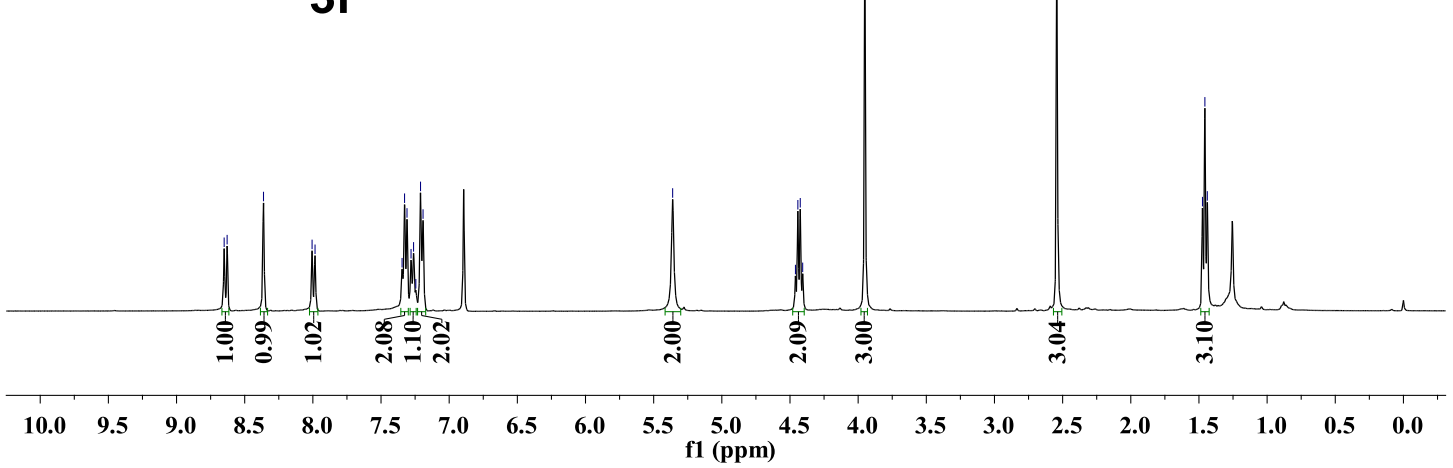

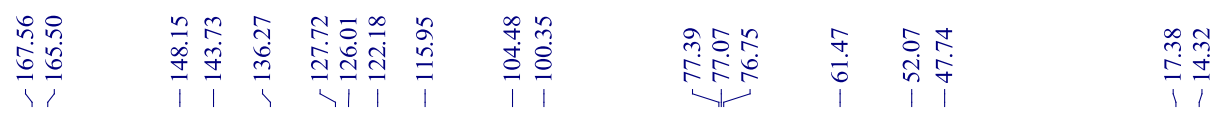<smiles>CCOC(=O)c1c(C)n(Cc2ccccc2)c(=O)n2c1cc1cc(C(=O)OC)ccc12</smiles>
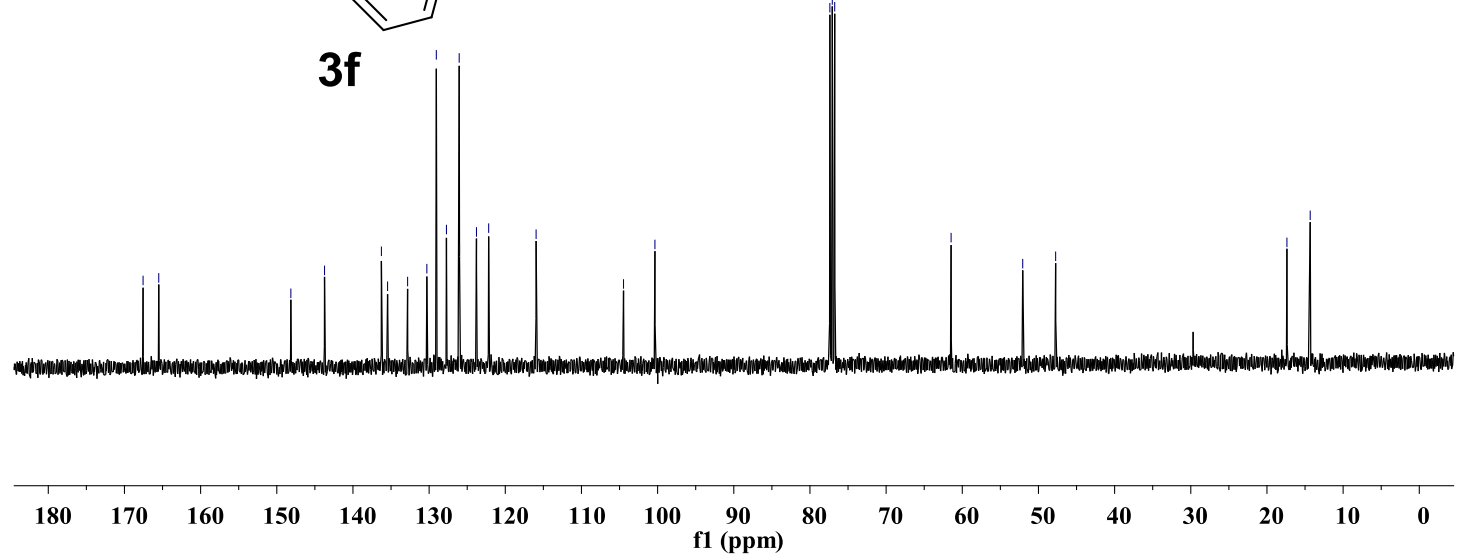
(7) The ${ }^{1} \mathrm{H}$ NMR and ${ }^{13} \mathrm{C}$ NMR spectrum for $\mathbf{3 g}$ (using $\mathrm{CDCl}_{3}$ as solvent)

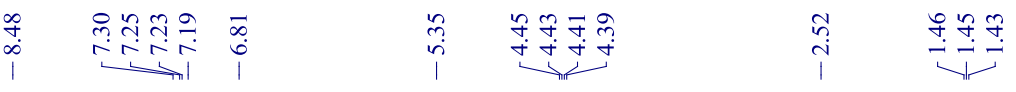<smiles>CCOC(=O)c1c(C)n(Cc2ccccc2)c(=O)n2c1cc1ccc(C)cc12</smiles>

$3 \mathrm{~g}$

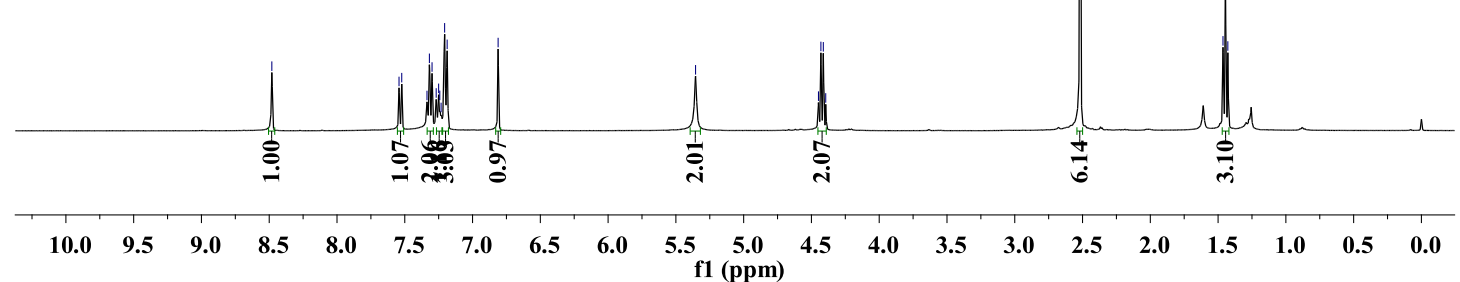

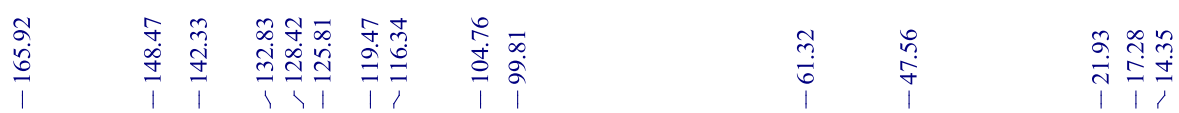<smiles>CCOC(=O)c1c(C)n(Cc2ccccc2)c(=O)n2c1cc1ccc(C)cc12</smiles>

$3 g$

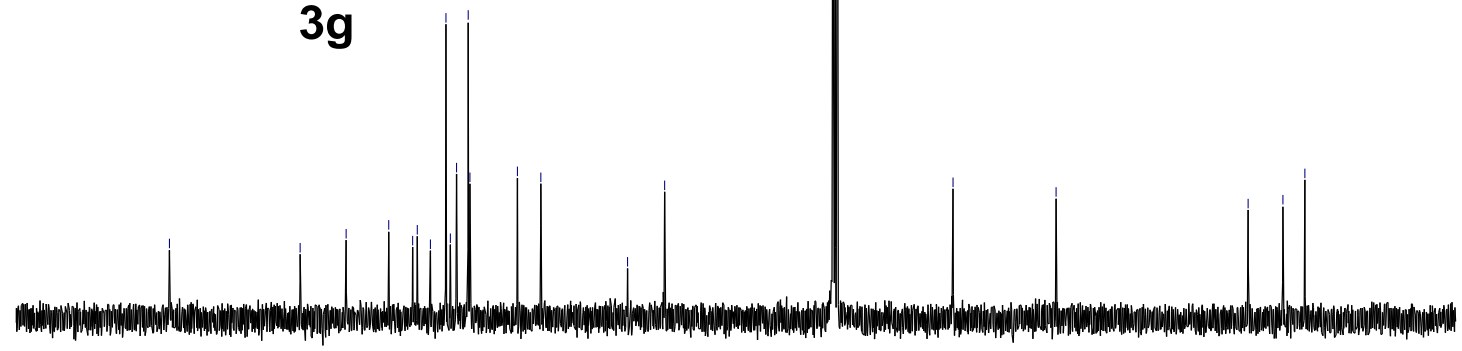

$\begin{array}{lllllllllllllllllll}180 & 170 & 160 & 150 & 140 & 130 & 120 & 110 & 100 \underset{\text { f1 }}{(\mathrm{ppm})} & 80 & 70 & 60 & 50 & 40 & 30 & 20 & 10 & 0\end{array}$ 
(8) The ${ }^{1} \mathrm{H}$ NMR and ${ }^{13} \mathrm{C}$ NMR spectrum for $\mathbf{3 h}$ (using $\mathrm{CDCl}_{3}$ as solvent)

$$
\text { 至 }
$$<smiles>CCOC(=O)c1c(C)n(Cc2ccccc2)c(=O)n2c1cc1ccc(Cl)cc12</smiles>

\section{$3 h$}<smiles>CCOC(=O)c1c(C)n(Cc2ccccc2)c(=O)n2c1cc1ccc(Cl)cc12</smiles>

\section{3h}

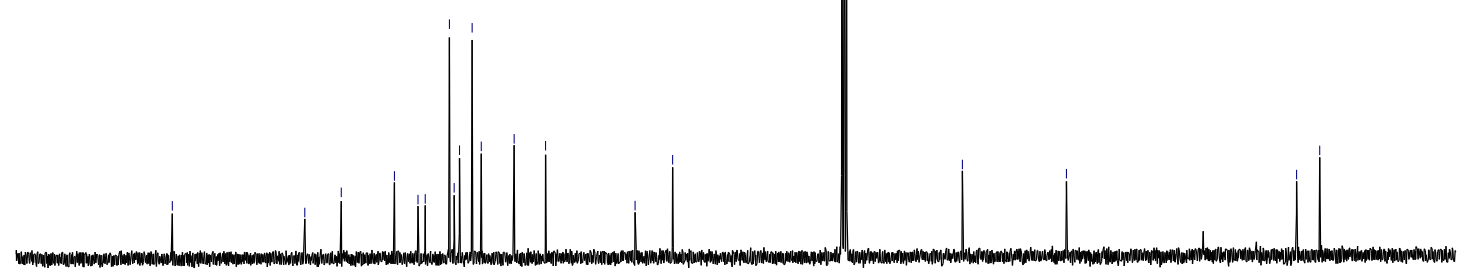

$\begin{array}{llllllllll}180 & 170 & 160 & 150 & 140 & 130 & 120 & 110 & 100 \underset{\text { f1 }}{(\mathrm{ppm})}\end{array}$ 
(9) The ${ }^{1} \mathrm{H}$ NMR and ${ }^{13} \mathrm{C}$ NMR spectrum for $3 \mathbf{i}$ (using $\mathrm{CDCl}_{3}$ as solvent)

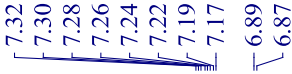

$$
\begin{aligned}
& \text { în }
\end{aligned}
$$

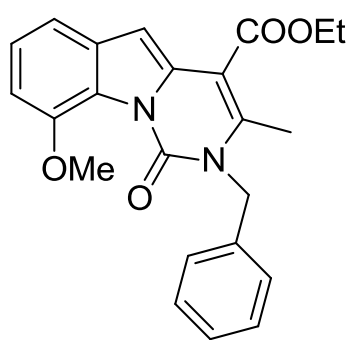

3i
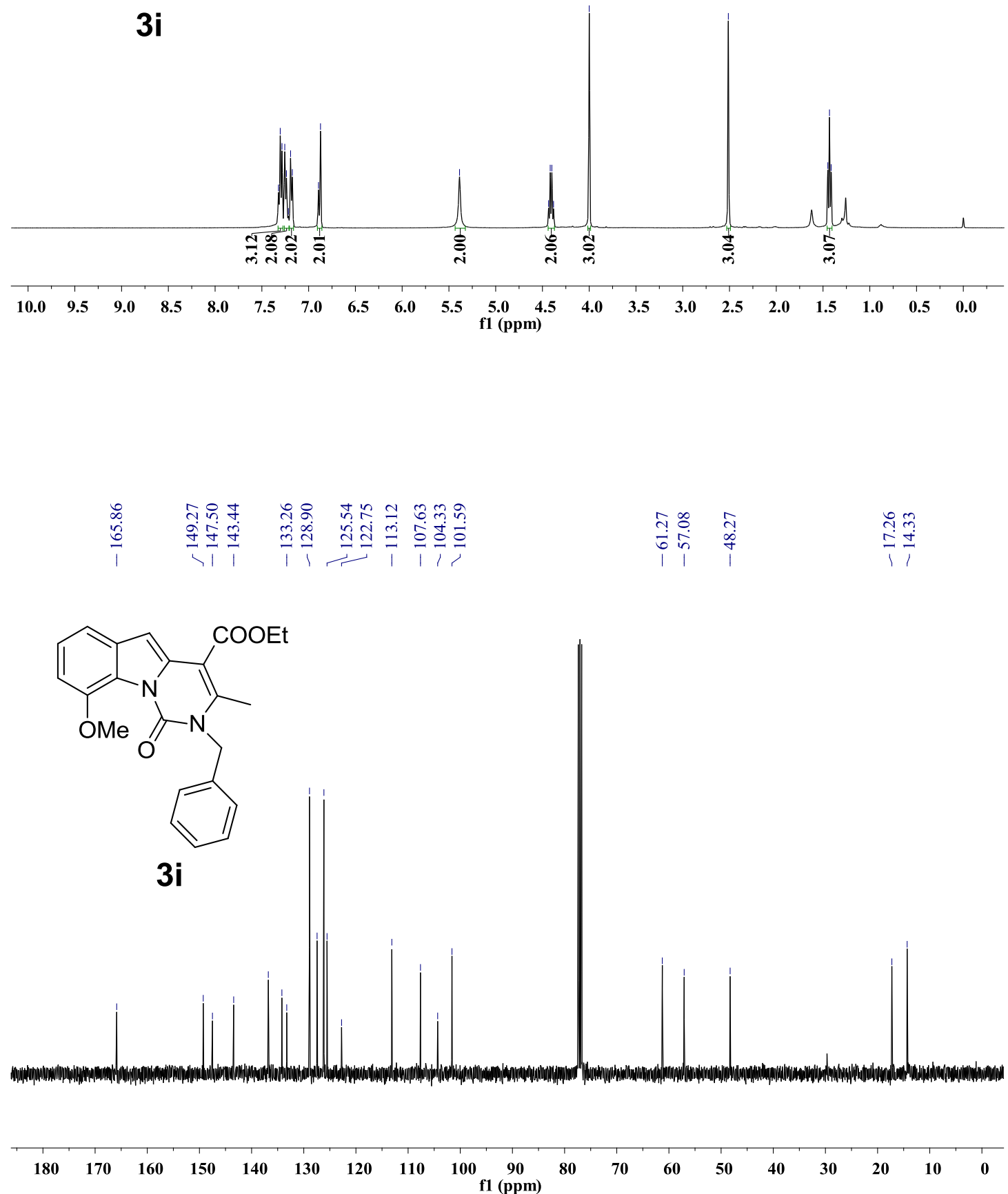
(10) The ${ }^{1} \mathrm{H}$ NMR and ${ }^{13} \mathrm{C}$ NMR spectrum for $\mathbf{3 j}$ (using $\mathrm{CDCl}_{3}$ as solvent)

$$
\text { لr }
$$<smiles>CCOC(=O)c1c(C)n(Cc2ccccc2)c(=O)n2c1cc1c(C)cccc12</smiles>

\section{3j}

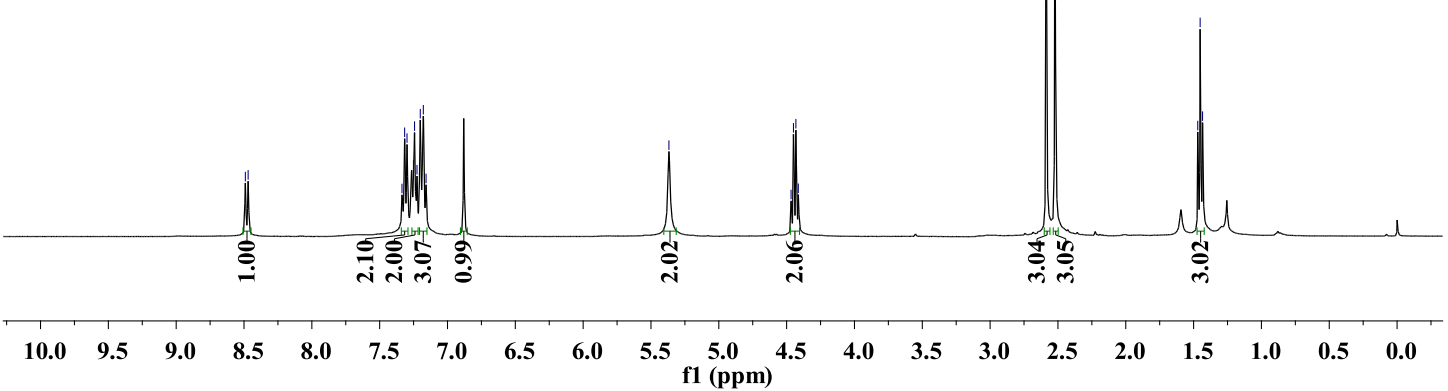<smiles>CCOC(=O)c1c(C)n(Cc2ccccc2)c(=O)n2c1cc1c(C)cccc12</smiles>

3j

180170

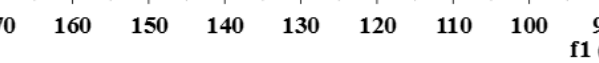
$\underset{\mathrm{f1}(\mathrm{ppm})}{90} 80$ 
(11) The ${ }^{1} \mathrm{H}$ NMR and ${ }^{13} \mathrm{C}$ NMR spectrum for $3 \mathbf{k}$ (using $\mathrm{CDCl}_{3}$ as solvent)

$$
\text { لy }
$$<smiles></smiles>

\section{3k}

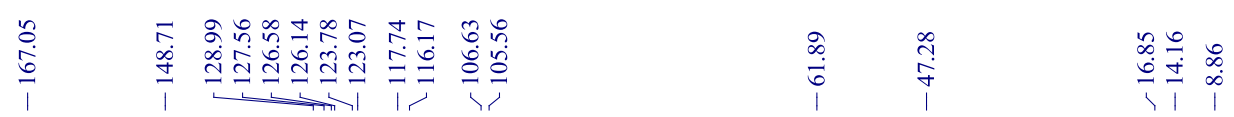<smiles>CCOC(=O)c1c(C)c2c(C)c3ccccc3n2c(=O)n1Cc1ccccc1</smiles>

3k

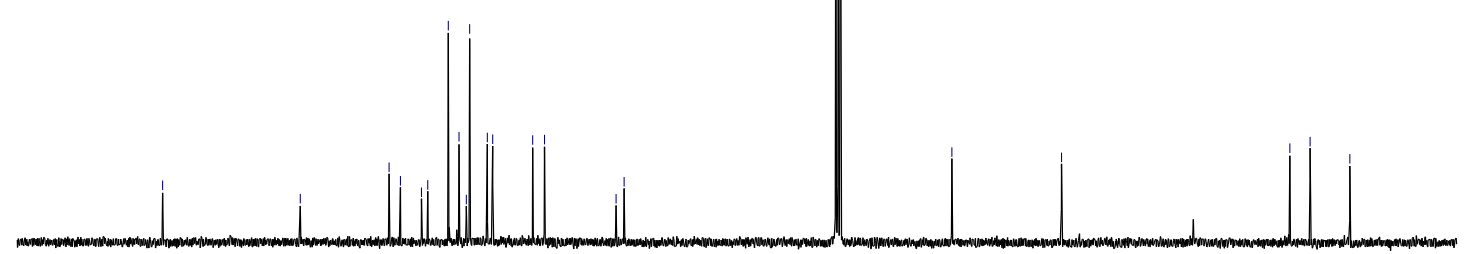

$\begin{array}{llllllllllllllllll}180 & 170 & 160 & 150 & 140 & 130 & 120 & 110 & 100 \underset{\text { f1 }}{\underset{(\mathrm{ppm})}{90}} & 80 & 70 & 60 & 50 & 40 & 30 & 20 & 10 & 0\end{array}$ 
(12) The ${ }^{1} \mathrm{H}$ NMR and ${ }^{13} \mathrm{C}$ NMR spectrum for $3 \mathbf{l}$ (using $\mathrm{CDCl}_{3}$ as solvent)

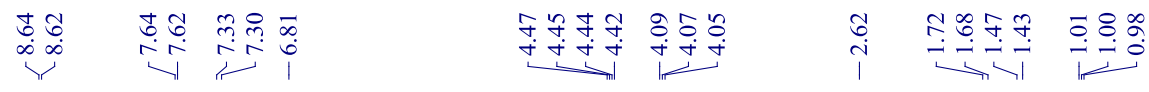<smiles>CCCCn1c(C)c(C(=O)OCC)c2cc3ccccc3n2c1=O</smiles>

\section{I}<smiles>CCCCn1c(C)c(C(=O)OCC)c2cc3ccccc3n2c1=O</smiles>

$180 \quad 170 \quad 160$

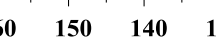

$\begin{array}{llll}130 & 120 & 110 & 10\end{array}$ $00 \underset{\text { f1 (ppm) }}{90} 80$ 
(13) The ${ }^{1} \mathrm{H}$ NMR and ${ }^{13} \mathrm{C}$ NMR spectrum for $\mathbf{3 m}$ (using $\mathrm{CDCl}_{3}$ as solvent)

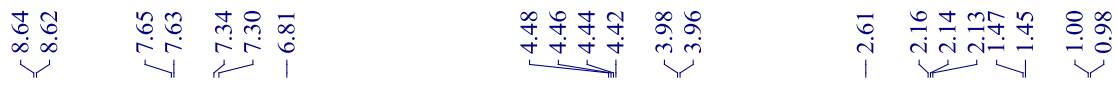<smiles>CCOC(=O)c1c(C)n(CC(C)C)c(=O)n2c1cc1ccccc12</smiles>

\section{$3 \mathrm{~m}$}

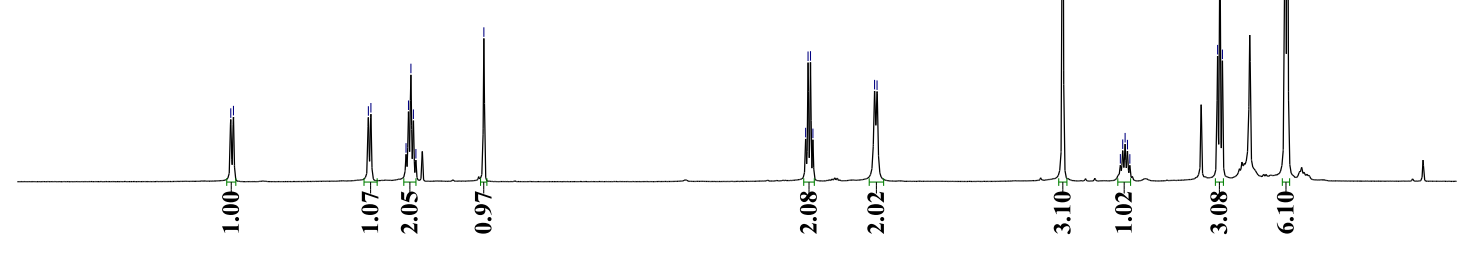

$\begin{array}{lllllllllllllllllllll}10.0 & 9.5 & 9.0 & 8.5 & 8.0 & 7.5 & 7.0 & 6.5 & 6.0 & 5.5 & \begin{array}{c}5.0 \\ \text { f1 }\end{array}(\mathrm{ppm}) & 4.5 & 4.0 & 3.5 & 3.0 & 2.5 & 2.0 & 1.5 & 1.0 & 0.5 & 0.0\end{array}$

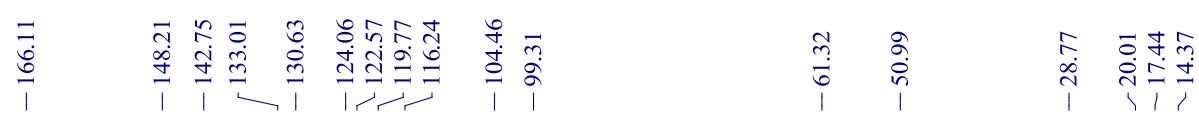<smiles>CCOC(=O)c1c(C)n(CC(C)C)c(=O)n2c1cc1ccccc12</smiles>

$3 \mathrm{~m}$ 
(14) The ${ }^{1} \mathrm{H}$ NMR and ${ }^{13} \mathrm{C}$ NMR spectrum for $3 \mathbf{n}$ (using $\mathrm{CDCl}_{3}$ as solvent)

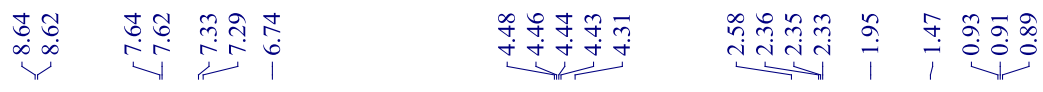<smiles>CCOC(=O)c1c(C)n(C(C)CC)c(=O)n2c1cc1ccccc12</smiles>

$3 n$

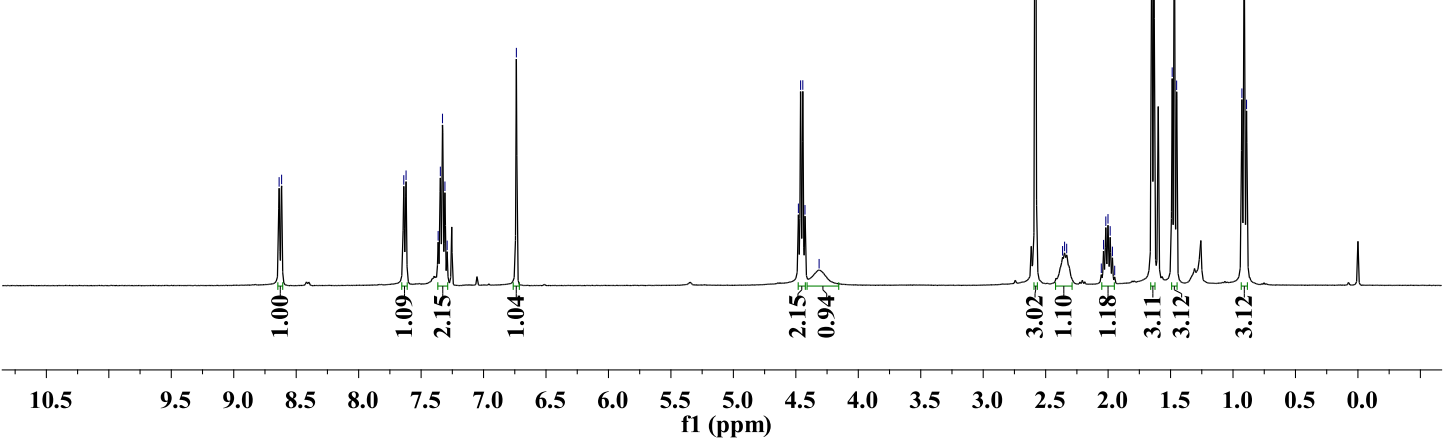

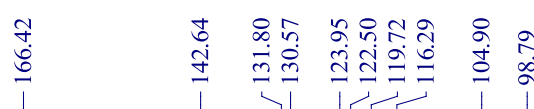

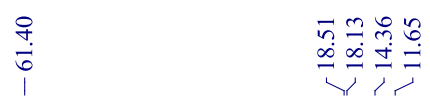<smiles>CCOC(=O)c1c(C)n(C(C)CC)c(=O)n2c1cc1ccccc12</smiles>

$3 n$

$\begin{array}{llll}180 & 170 & 160 & 150\end{array}$ $\begin{array}{llll}150 & 140 & 130 & 120\end{array}$ $120 \quad 110 \quad 100 \underset{\text { f1 (ppm) }}{90} 8$ $\begin{array}{lllllllll}80 & 70 & 60 & 50 & 40 & 30 & 20 & 10 & 0\end{array}$ 
(15) The ${ }^{1} \mathrm{H}$ NMR and ${ }^{13} \mathrm{C}$ NMR spectrum for 30 (using $\mathrm{CDCl}_{3}$ as solvent)

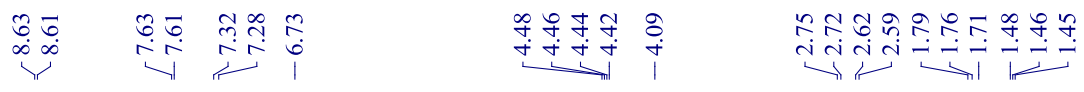<smiles>CCOC(=O)c1c(C)n(C2CCCCC2)c(=O)n2c1cc1ccccc12</smiles>

30

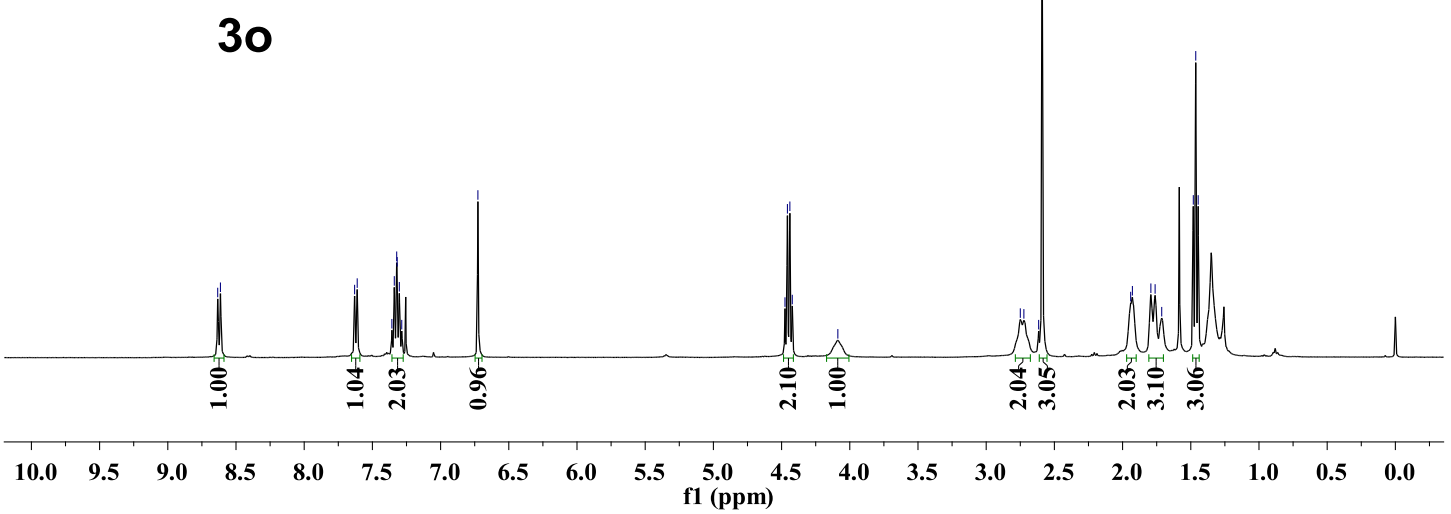<smiles>CCOC(=O)c1c(C)n(C2CCCCC2)c(=O)n2c1cc1ccccc12</smiles>

30 
(16) The ${ }^{1} \mathrm{H}$ NMR and ${ }^{13} \mathrm{C}$ NMR spectrum for $\mathbf{3 p}$ (using $\mathrm{CDCl}_{3}$ as solvent)

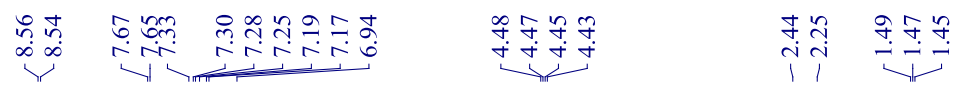

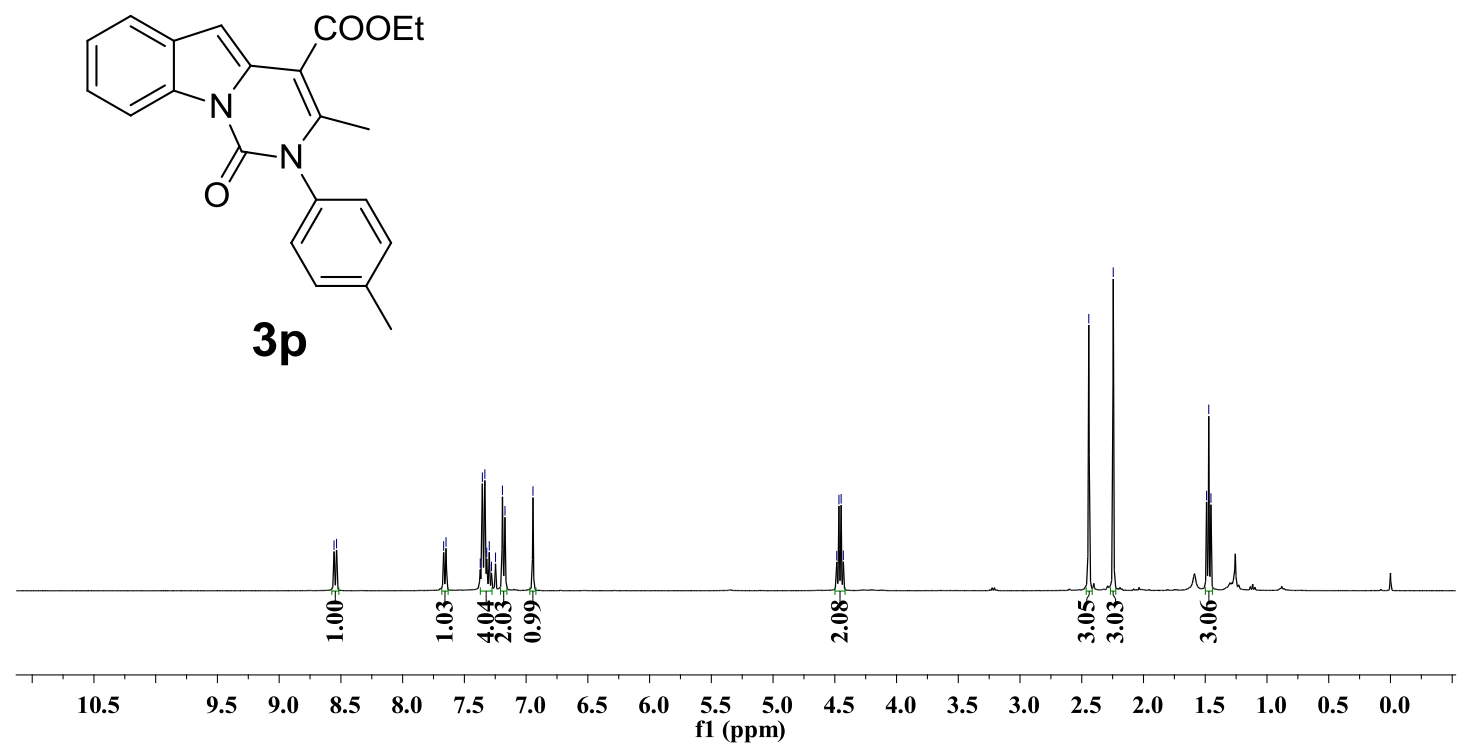

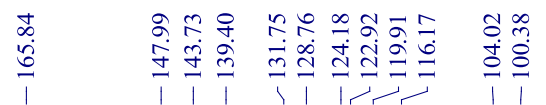
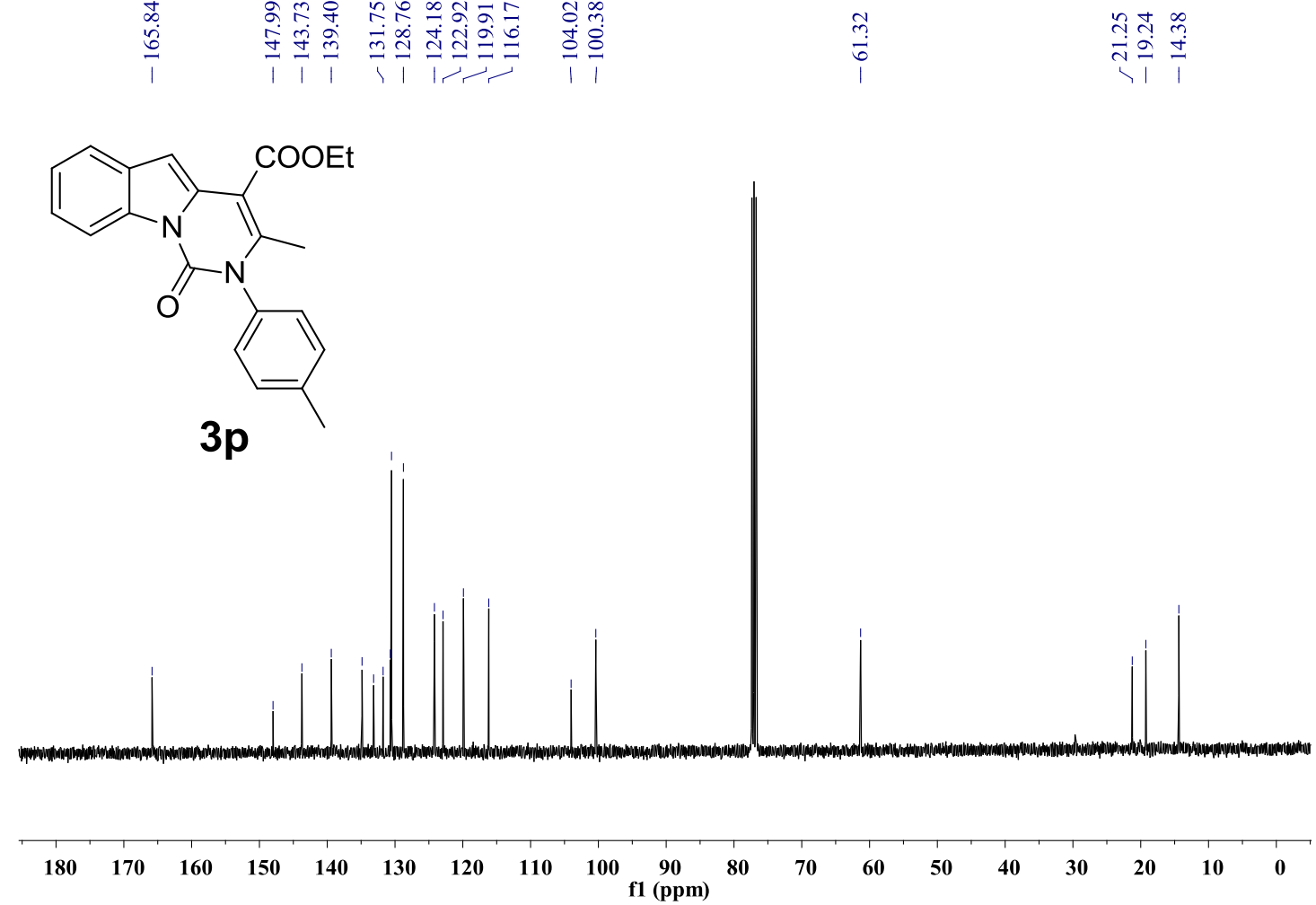
(17) The ${ }^{1} \mathrm{H}$ NMR and ${ }^{13} \mathrm{C}$ NMR spectrum for $\mathbf{3 q}$ (using $\mathrm{CDCl}_{3}$ as solvent)

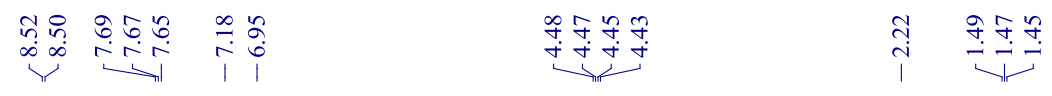<smiles>CCOC(=O)c1c(C)n(-c2ccc(Br)cc2)c(=O)n2c1cc1ccccc12</smiles>

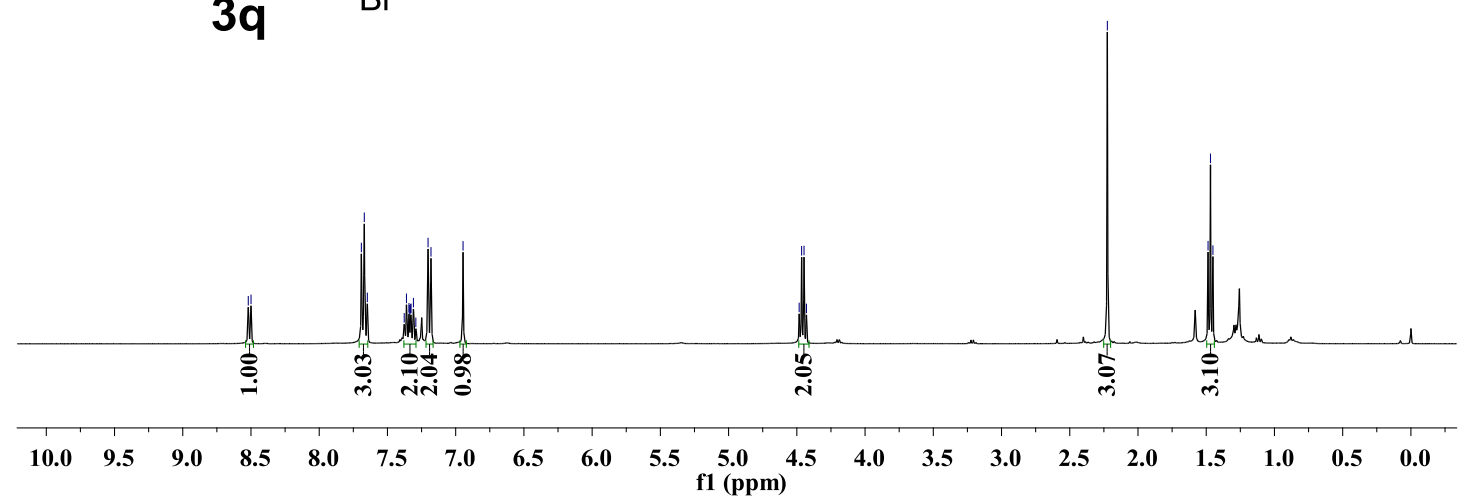

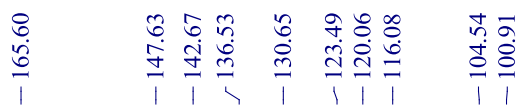

$\underset{\text { ষ্ণ }}{\text { i }}$

$\stackrel{\stackrel{\sim}{\sim}}{\stackrel{m}{ \pm}}$<smiles>CCOC(=O)c1c(C)n(-c2ccc(Br)cc2)c(=O)n2c1cc1ccccc12</smiles> 
(18) The ${ }^{1} \mathrm{H}$ NMR and ${ }^{13} \mathrm{C}$ NMR spectrum for $3 \mathbf{r}$ (using $\mathrm{CDCl}_{3}$ as solvent)

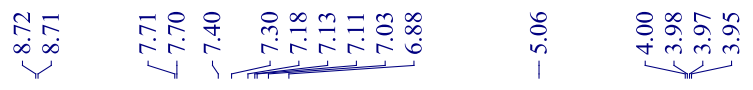<smiles>CCOC(=O)c1c(-c2ccccc2)n(Cc2ccccc2)c(=O)n2c1cc1ccccc12</smiles>

$3 r$

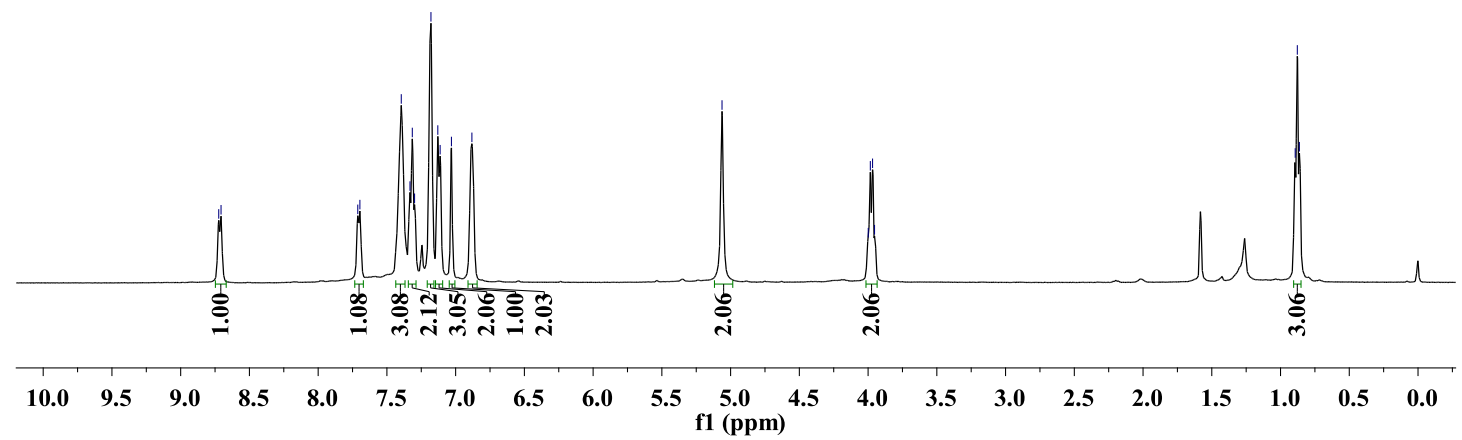

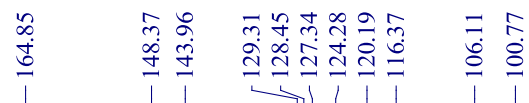

$\begin{array}{lll}8 & \infty \\ 0 & \infty \\ 0 & +\infty \\ 1 & 1\end{array}$

$\underset{\substack{1 \\ m}}{\stackrel{3}{m}}$<smiles>CCOC(=O)c1c(-c2ccccc2)n(Cc2ccccc2)c(=O)n2c1cc1ccccc12</smiles>

$3 r$

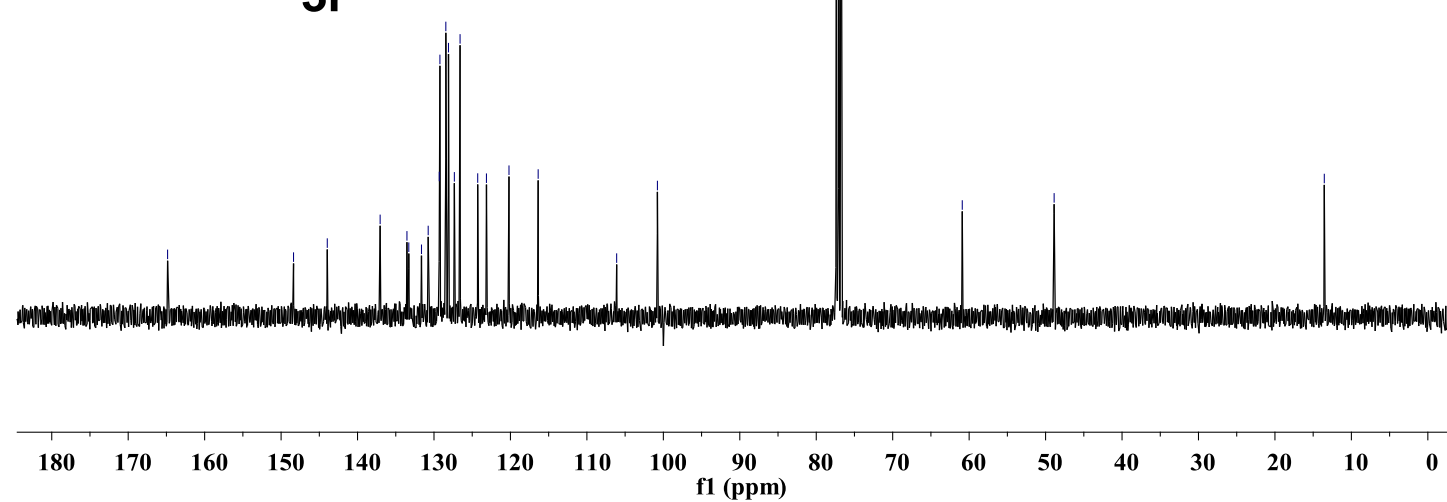


(19) The ${ }^{1} \mathrm{H}$ NMR and ${ }^{13} \mathrm{C}$ NMR spectrum for $3 \mathbf{s}$ (using $\mathrm{CDCl}_{3}$ as solvent)<smiles>CCOC(=O)c1c(CCP)n(Cc2ccccc2)c(=O)n2c1cc1ccccc12</smiles>

3s

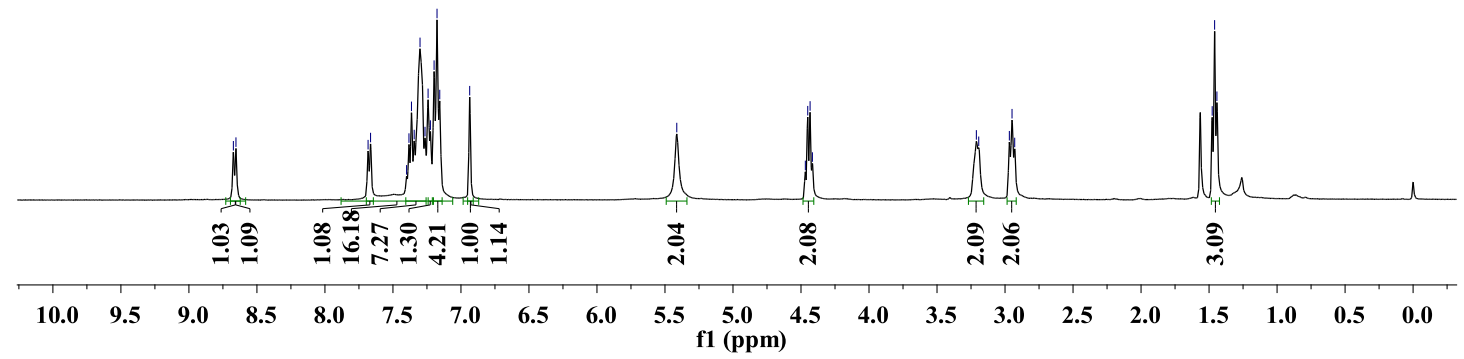

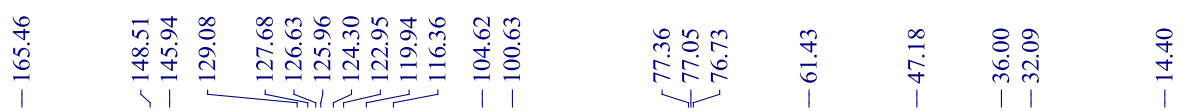<smiles>CCOC(=O)c1c(CCc2ccccc2)n(Cc2ccccc2)c(=O)n2c1cc1ccccc12</smiles>

3s

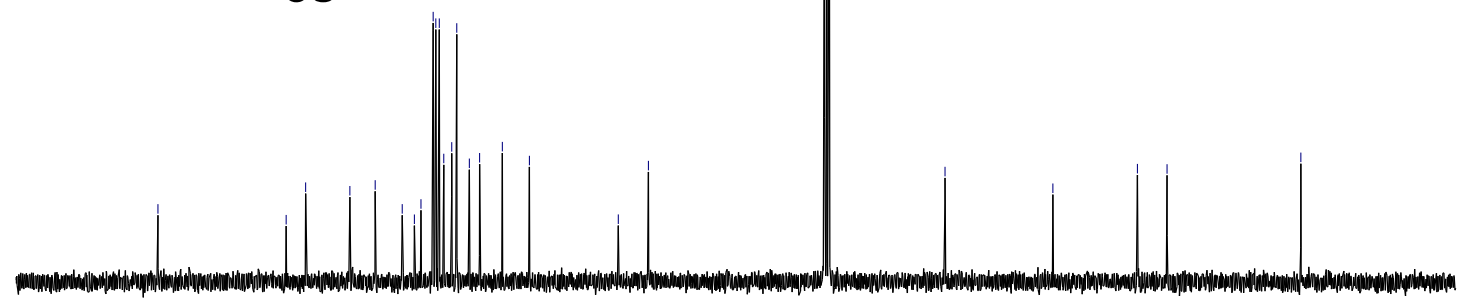


(20) The ${ }^{1} \mathrm{H}$ NMR and ${ }^{13} \mathrm{C}$ NMR spectrum for $\mathbf{3 t}$ (using $\mathrm{CDCl}_{3}$ as solvent)

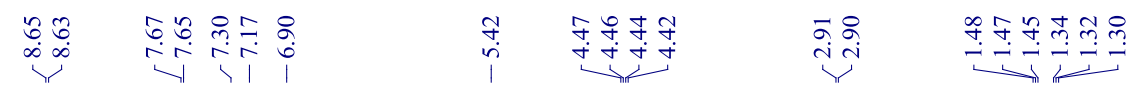<smiles>CCOC(=O)c1c(CC)n(Cc2ccccc2)c(=O)n2c1cc1ccccc12</smiles>

$3 \mathbf{t}$

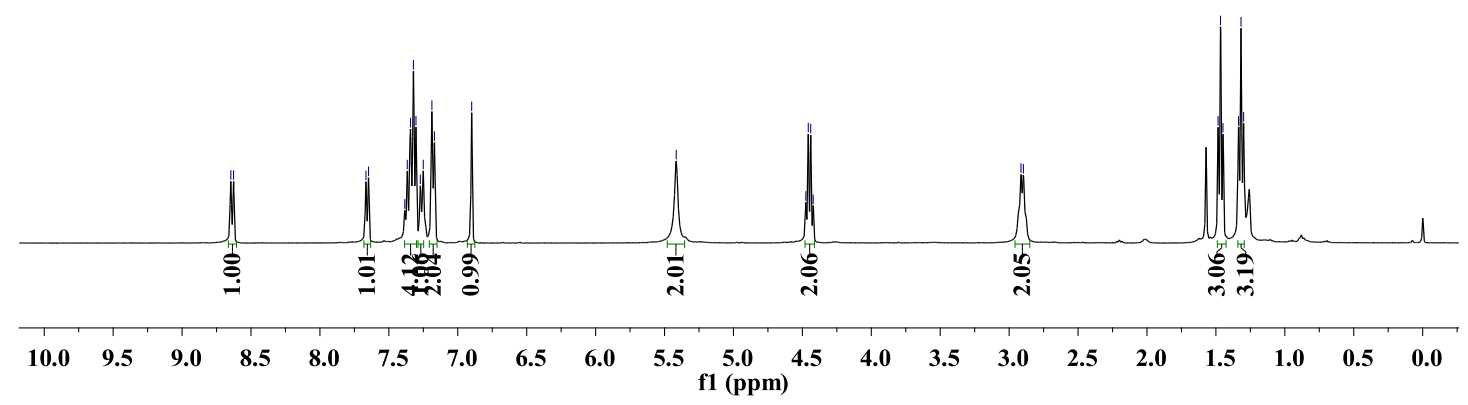<smiles>CCOC(=O)c1c(CC)n(Cc2ccccc2)c(=O)n2c1cc1ccccc12</smiles>

$3 t$

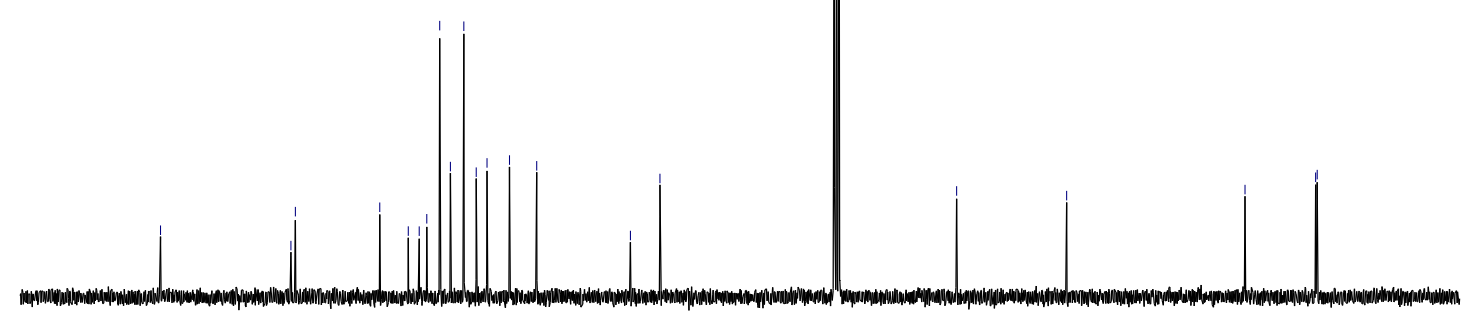


(21) The ${ }^{1} \mathrm{H}$ NMR and ${ }^{13} \mathrm{C}$ NMR spectrum for $3 \mathbf{u}$ (using $\mathrm{CDCl}_{3}$ as solvent)

$$
\text { 官 }
$$<smiles>COCc1c(C(=O)OC)n(Cc2ccccc2)c(=O)n2c1cc1ccccc12</smiles>

\section{$3 u$}

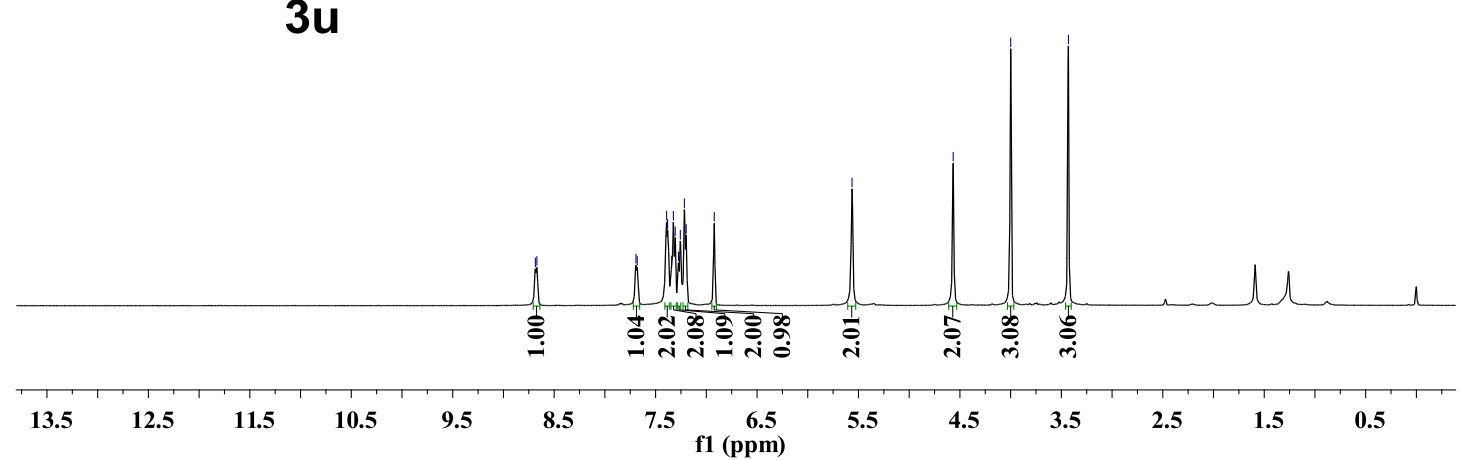

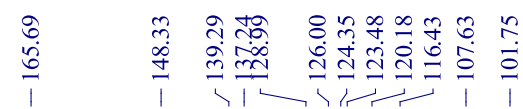

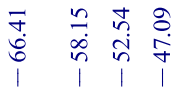<smiles>COCc1c(C(=O)OC)n(Cc2ccccc2)c(=O)n2c1cc1ccccc12</smiles>

$3 u$

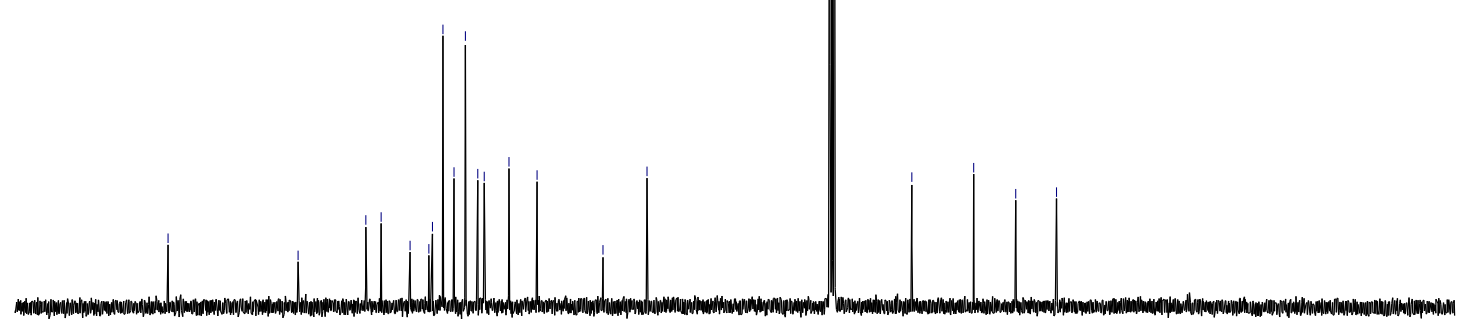

$\begin{array}{lllllllllllllllllll}180 & 170 & 160 & 150 & 140 & 130 & 120 & 110 & 100 & \underset{f 1}{90} & 80 & 70 & 60 & 50 & 40 & 30 & 20 & 10 & 0\end{array}$ 
(22) The ${ }^{1} \mathrm{H}$ NMR and ${ }^{13} \mathrm{C}$ NMR spectrum for $\mathbf{3 v}$ (using $\mathrm{CDCl}_{3}$ as solvent)

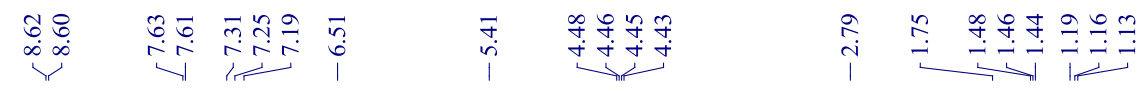<smiles>CCOC(=O)c1c(C2CCCCC2)n(Cc2ccccc2)c(=O)n2c1cc1ccccc12</smiles>

\section{3v}

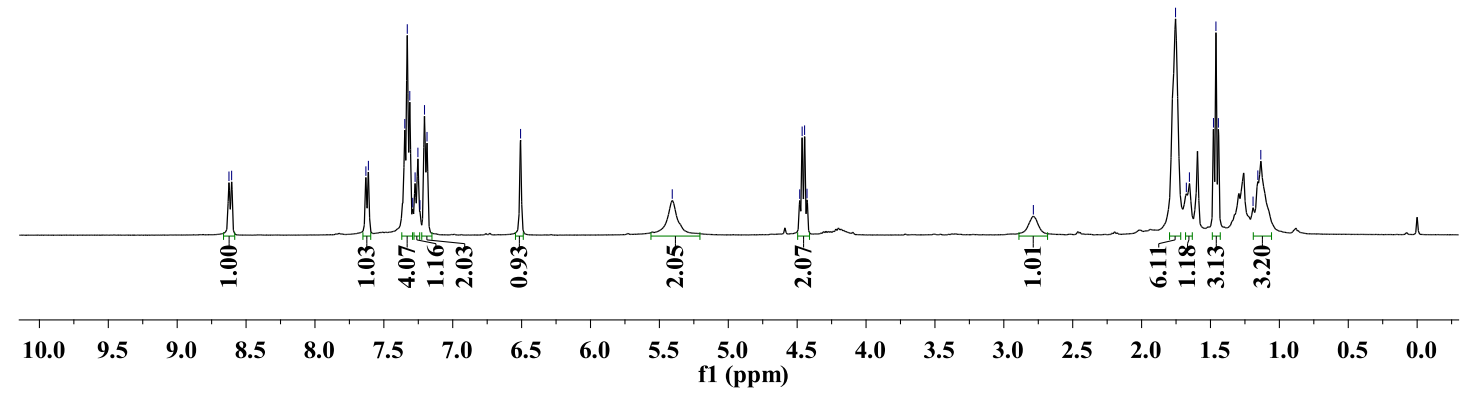

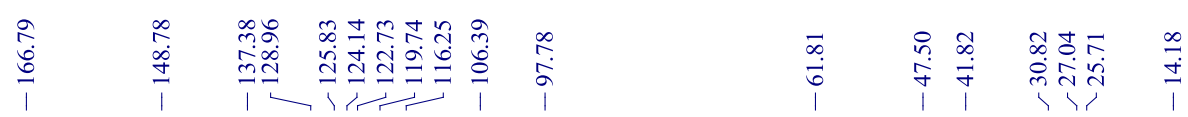<smiles>CCOC(=O)c1c(C2CCCCC2)n(Cc2ccccc2)c(=O)n2c1cc1ccccc12</smiles>

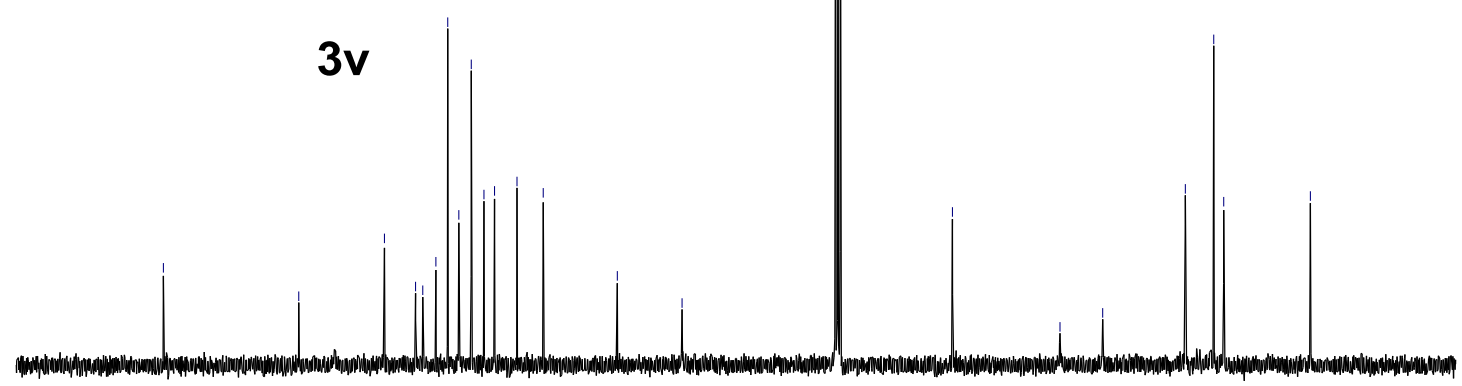

$\begin{array}{lllllllllllllllllll}180 & 170 & 160 & 150 & 140 & 130 & 120 & 110 & 100 \underset{\mathrm{f} 1(\mathrm{ppm})}{90} & 80 & 70 & 60 & 50 & 40 & 30 & 20 & 10 & 0\end{array}$ 
(23) The ${ }^{1} \mathrm{H}$ NMR and ${ }^{13} \mathrm{C}$ NMR spectrum for $3 \mathbf{w}$ (using $\mathrm{CDCl}_{3}$ as solvent)

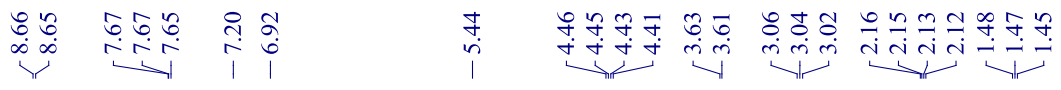<smiles>CCOC(=O)c1c(CCCCl)n(Cc2ccccc2)c(=O)n2c1cc1ccccc12</smiles>

\section{$3 w$}

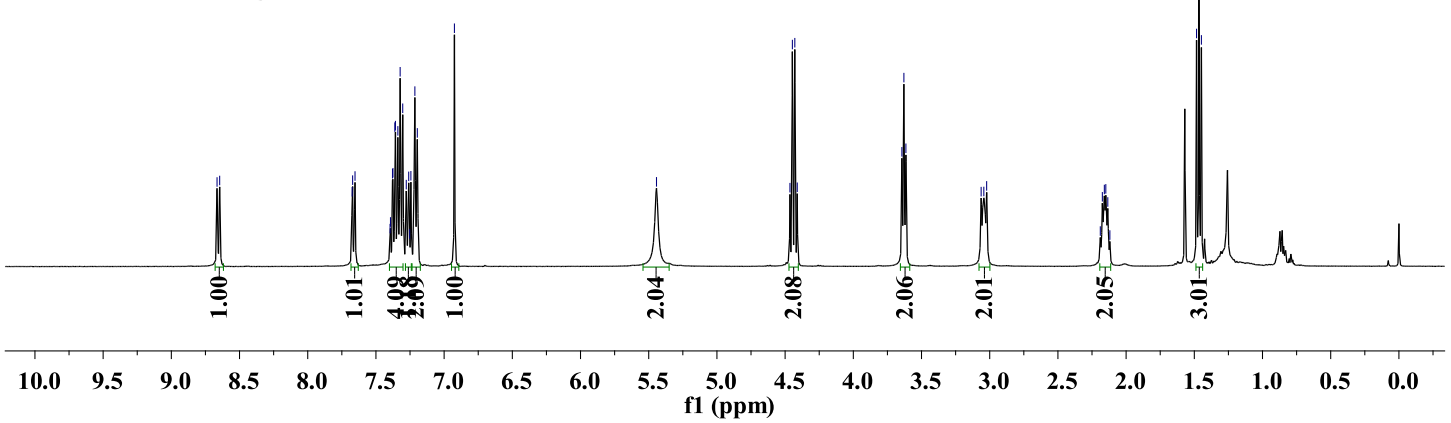<smiles>CCOC(=O)c1c(CCCCl)n(Cc2ccccc2)c(=O)n2c1cc1ccccc12</smiles>

\section{$3 w$}

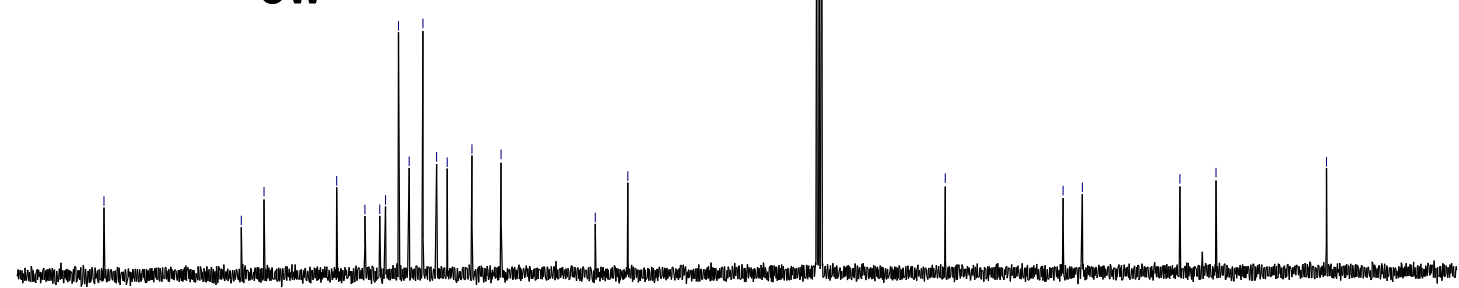

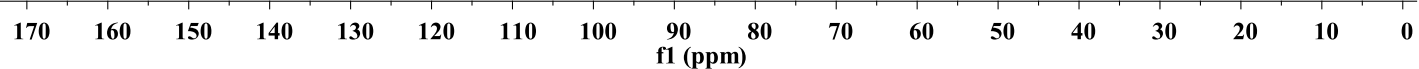


(24) The ${ }^{1} \mathrm{H}$ NMR and ${ }^{13} \mathrm{C}$ NMR spectrum for $\mathbf{3 x}$ (using $\mathrm{CDCl}_{3}$ as solvent)

$$
\text { 丩্ }
$$<smiles>CC(=O)c1c(C)n(Cc2ccccc2)c(=O)n2c1cc1ccccc12</smiles>

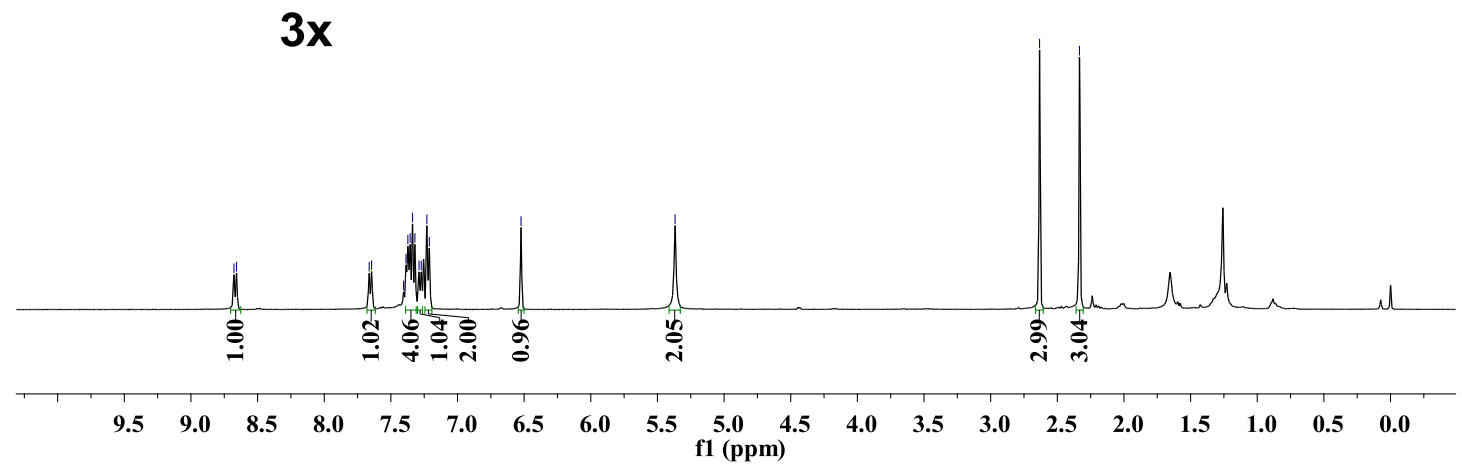

$\infty$
$\stackrel{2}{2}$
1<smiles>CC(=O)c1c(C)n(Cc2ccccc2)c(=O)n2c1cc1ccccc12</smiles>

$3 x$

$\begin{array}{llllllllllll}210 & 200 & 190 & 180 & 170 & 160 & 150 & 140 & 130 & 120 & \begin{array}{l}110 \\ \text { f1 (ppm) }\end{array}\end{array}$ 
(25) The ${ }^{1} \mathrm{H}$ NMR and ${ }^{13} \mathrm{C}$ NMR spectrum for $\mathbf{3 y}$ (using $\mathrm{CDCl}_{3}$ as solvent)

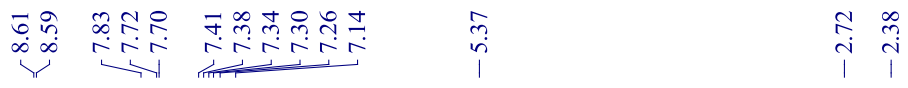<smiles>Cc1ccc(S(=O)(=O)c2c(C)n(Cc3ccccc3)c(=O)n3c2cc2ccccc23)cc1</smiles>

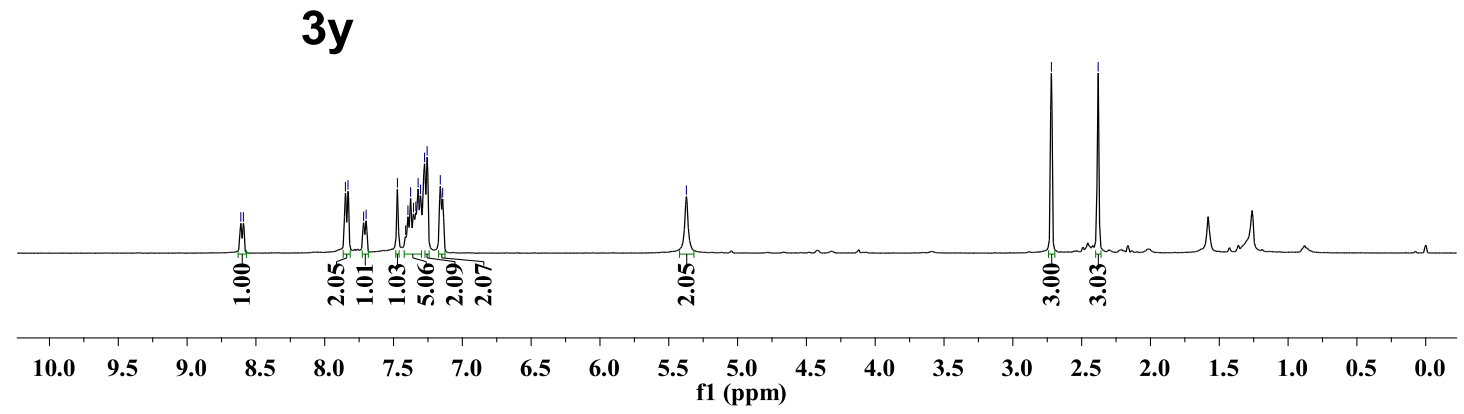

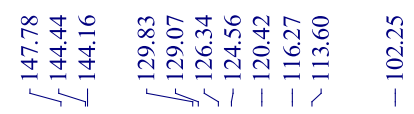

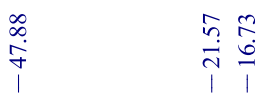<smiles>Cc1ccc(S(=O)(=O)c2c(C)n(Cc3ccccc3)c(=O)n3c2cc2ccccc23)cc1</smiles>

3y

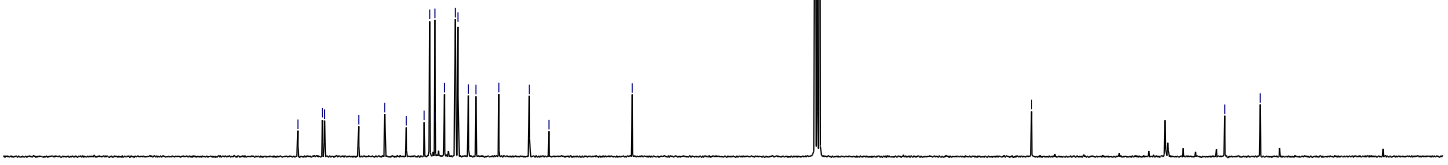

$\begin{array}{lllllllllllllllllll}180 & 170 & 160 & 150 & 140 & 130 & 120 & 110 & 100 \underset{\text { f1 } \underset{(\mathrm{ppm})}{90}}{80} & 70 & 60 & 50 & 40 & 30 & 20 & 10 & 0\end{array}$ 
(26) The ${ }^{1} \mathrm{H}$ NMR and ${ }^{13} \mathrm{C}$ NMR spectrum for $\mathbf{3 z}$ (using $\mathrm{CDCl}_{3}$ as solvent)

$$
\text { i }
$$<smiles>CCOC(=O)c1c(C)n(Cc2ccccc2)c(=O)n2cccc12</smiles>

$3 z$

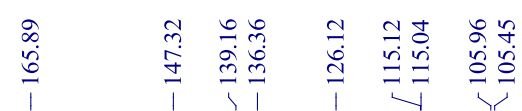<smiles>CCOC(=O)c1c(C)n(Cc2ccccc2)c(=O)n2cccc12</smiles>

$3 z$

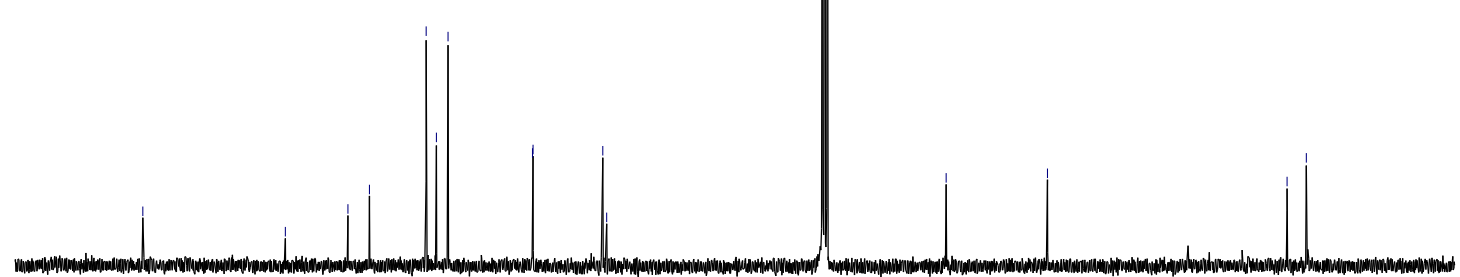


(27) The ${ }^{1} \mathrm{H}$ NMR and ${ }^{13} \mathrm{C}$ NMR spectrum for 3a-1 (using d-DMSO as solvent)

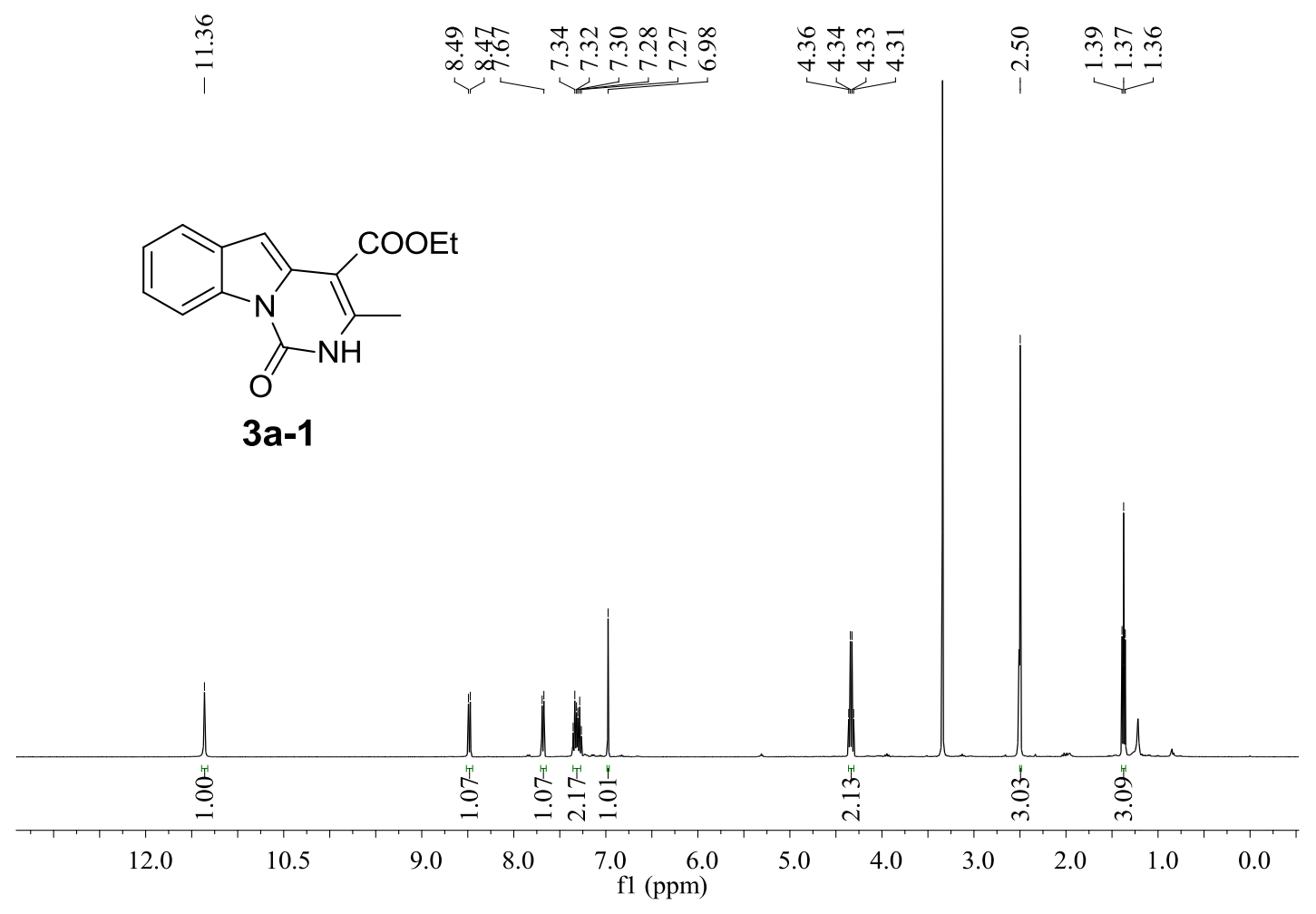

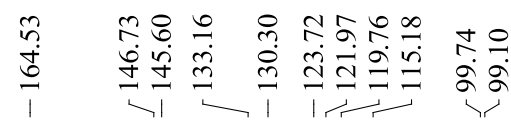
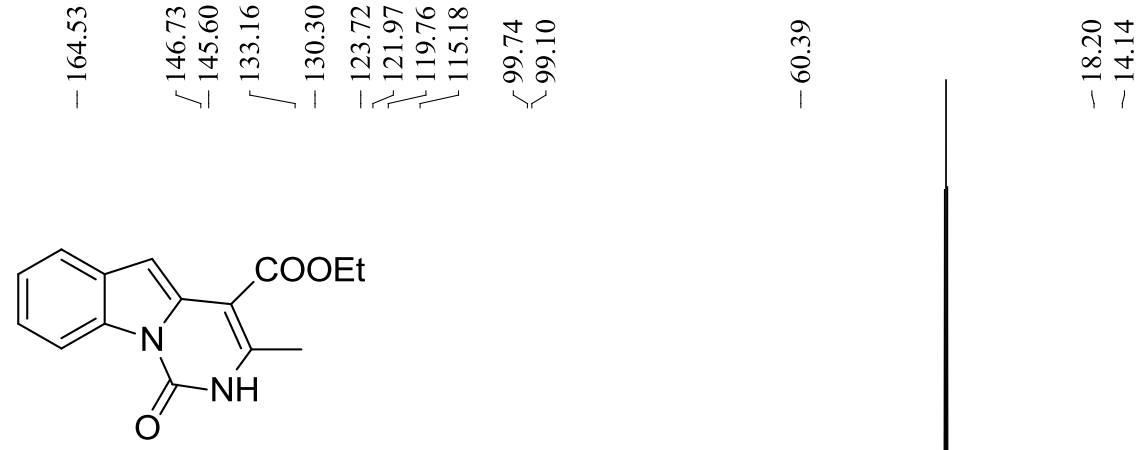

3a-1

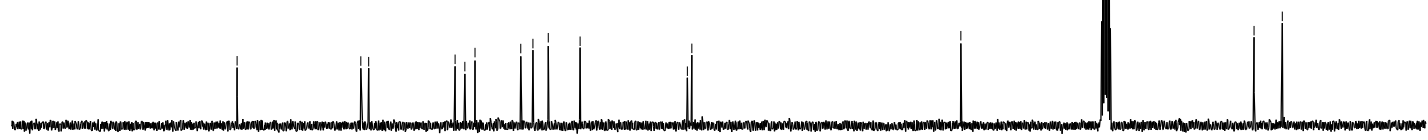

$\begin{array}{lllllllllllllll}190 & 170 & 150 & 130 & 110 & \begin{array}{r}90 \\ \mathrm{fl}(\mathrm{ppm})\end{array} & 80 & 70 & 60 & 50 & 40 & 30 & 20 & 10 & 0\end{array}$ 
(28) The ${ }^{1} \mathrm{H}$ NMR and ${ }^{13} \mathrm{C}$ NMR spectrum for 3a-2 (using d-DMSO as solvent)

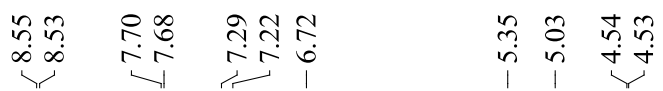

3a-2

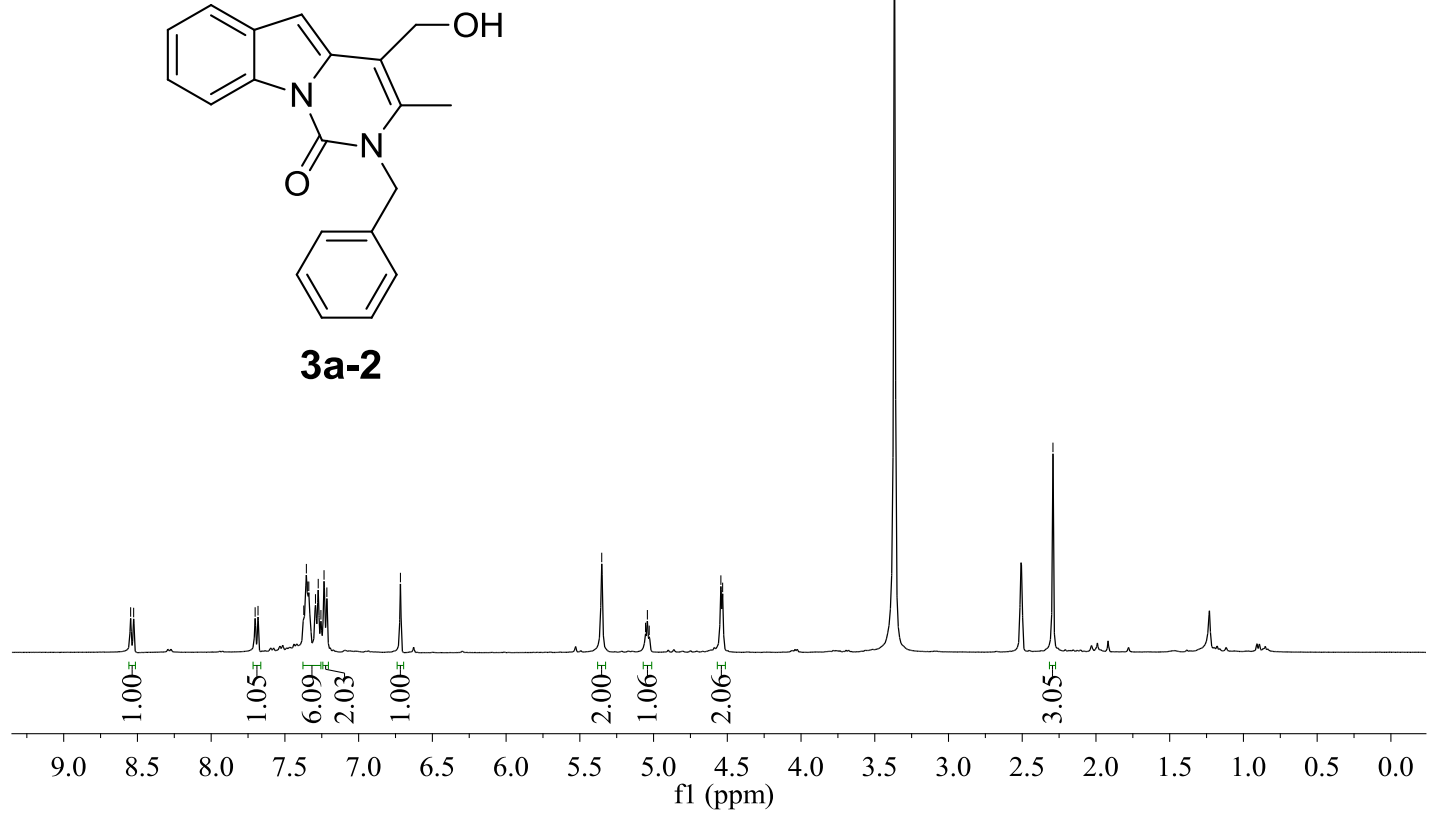

กิ

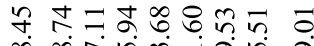

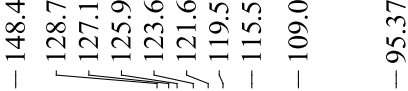

$\underset{\substack{\infty \\ i}}{i}$

$\stackrel{\widetilde{\alpha}}{\dot{ \pm}}$<smiles>Cc1c(CO)c2cc3ccccc3n2c(=O)n1Cc1ccccc1</smiles>

3a-2

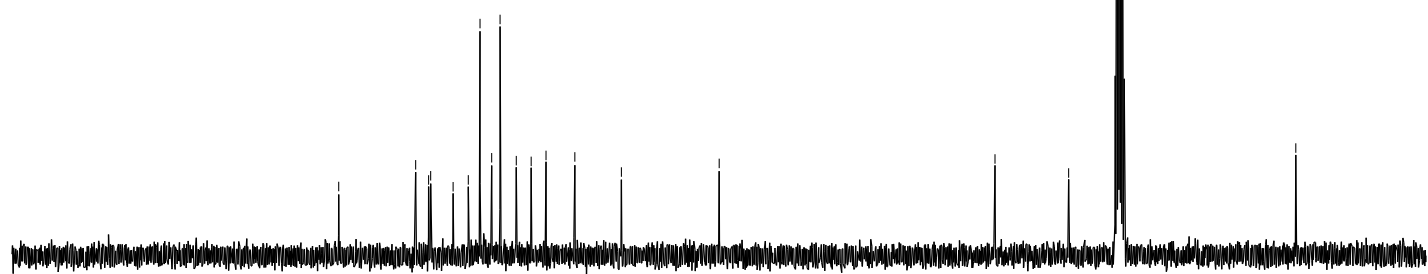

190

170

150

130

110

fl (ppm)

$\begin{array}{lllllll}60 & 50 & 40 & 30 & 20 & 10 & 0\end{array}$ 
(29) The ${ }^{1} \mathrm{H}$ NMR and ${ }^{13} \mathrm{C}$ NMR spectrum for $\mathbf{3 a - 3}$ (using $\mathrm{CDCl}_{3}$ as solvent)

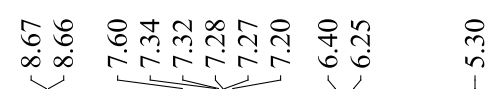

$\stackrel{\text { in }}{1}$

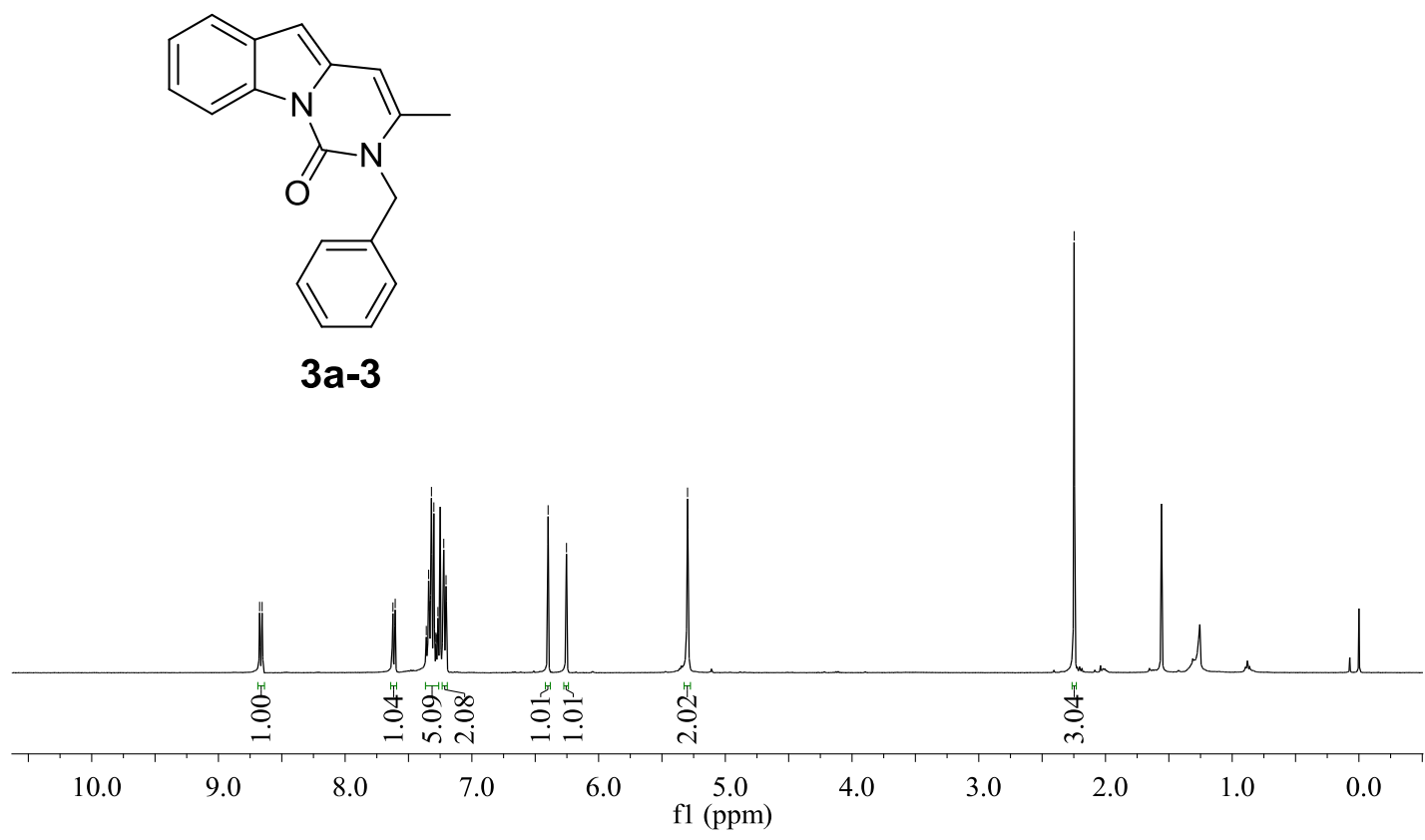

3a-3

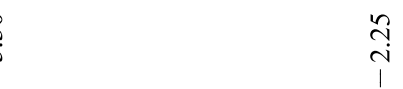

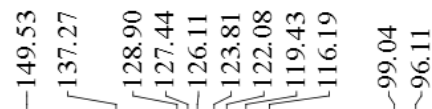

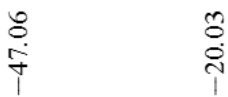

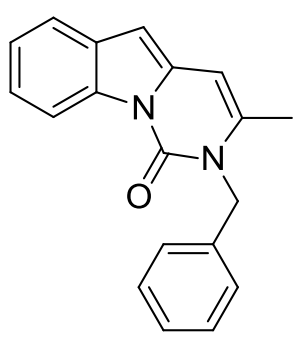

3a-3

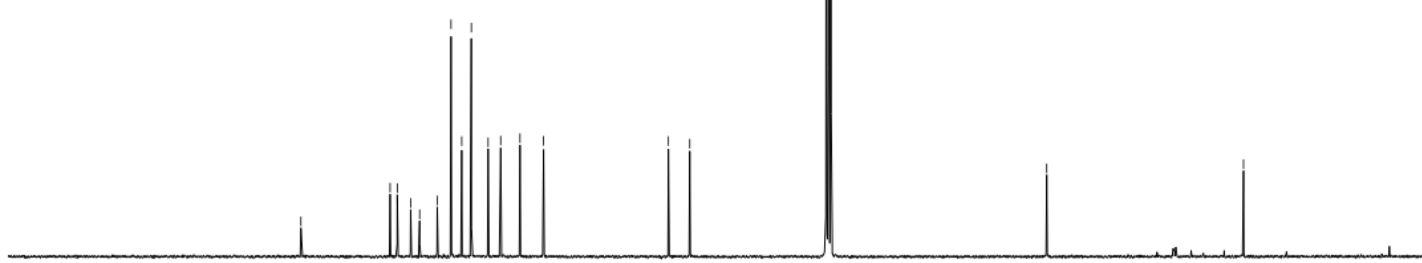

$\begin{array}{lllllllllllllllllll}180 & 170 & 160 & 150 & 140 & 130 & 120 & 110 & 100 & 90 & 80 & 70 & 60 & 50 & 40 & 30 & 20 & 10 & 0\end{array}$ f1 (ppm) 
(30) The ${ }^{1} \mathrm{H}$ NMR and ${ }^{13} \mathrm{C}$ NMR spectrum for $\mathbf{3 a}-\mathbf{4}$ (using $\mathrm{CDCl}_{3}$ as solvent)

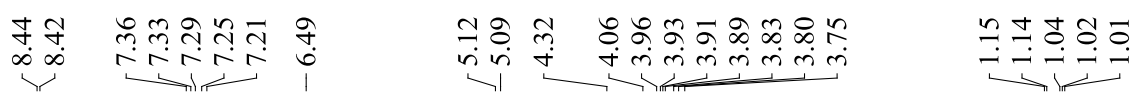<smiles>CCOC(=O)C1c2cc3ccccc3n2C(=O)N(Cc2ccccc2)C1C</smiles>

3a-4

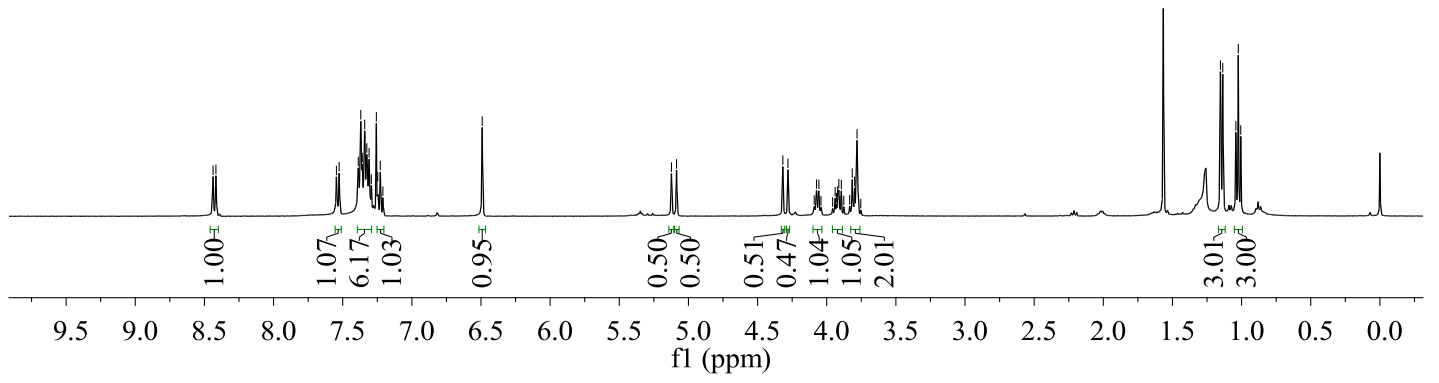

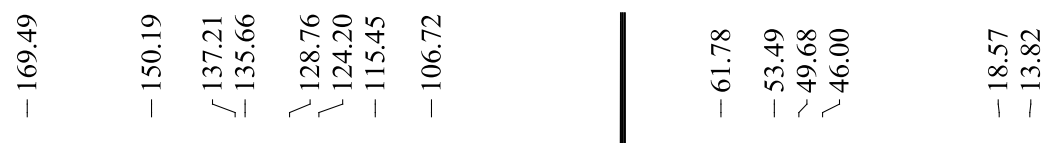<smiles>CCOC(=O)C1c2cc3ccccc3n2C(=O)N(Cc2ccccc2)C1C</smiles>

$3 a-4$

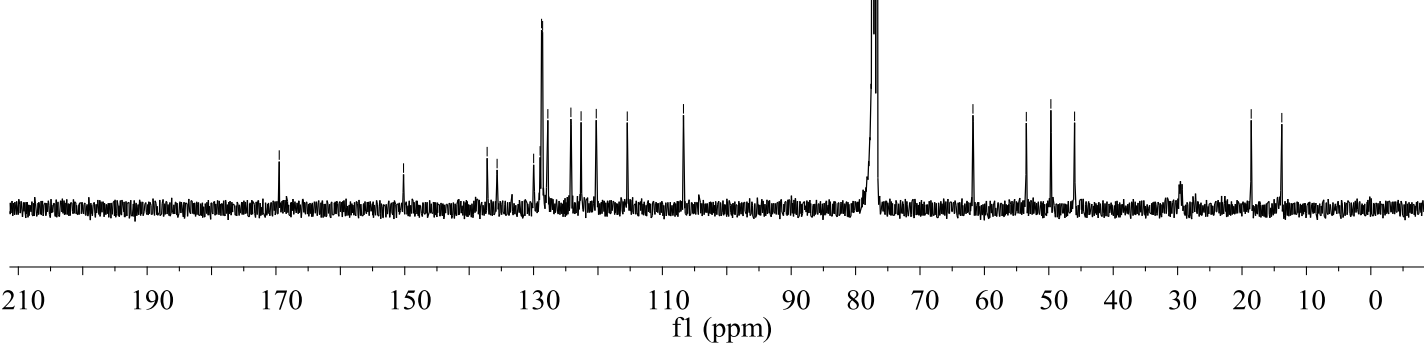


(31) The ${ }^{1} \mathrm{H}$ NMR and ${ }^{13} \mathrm{C}$ NMR spectrum for $\mathbf{d - 3 a}$ (using $\mathrm{CDCl}_{3}$ as solvent)

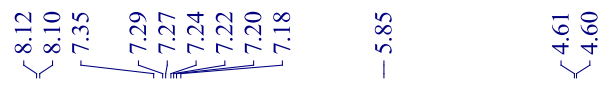
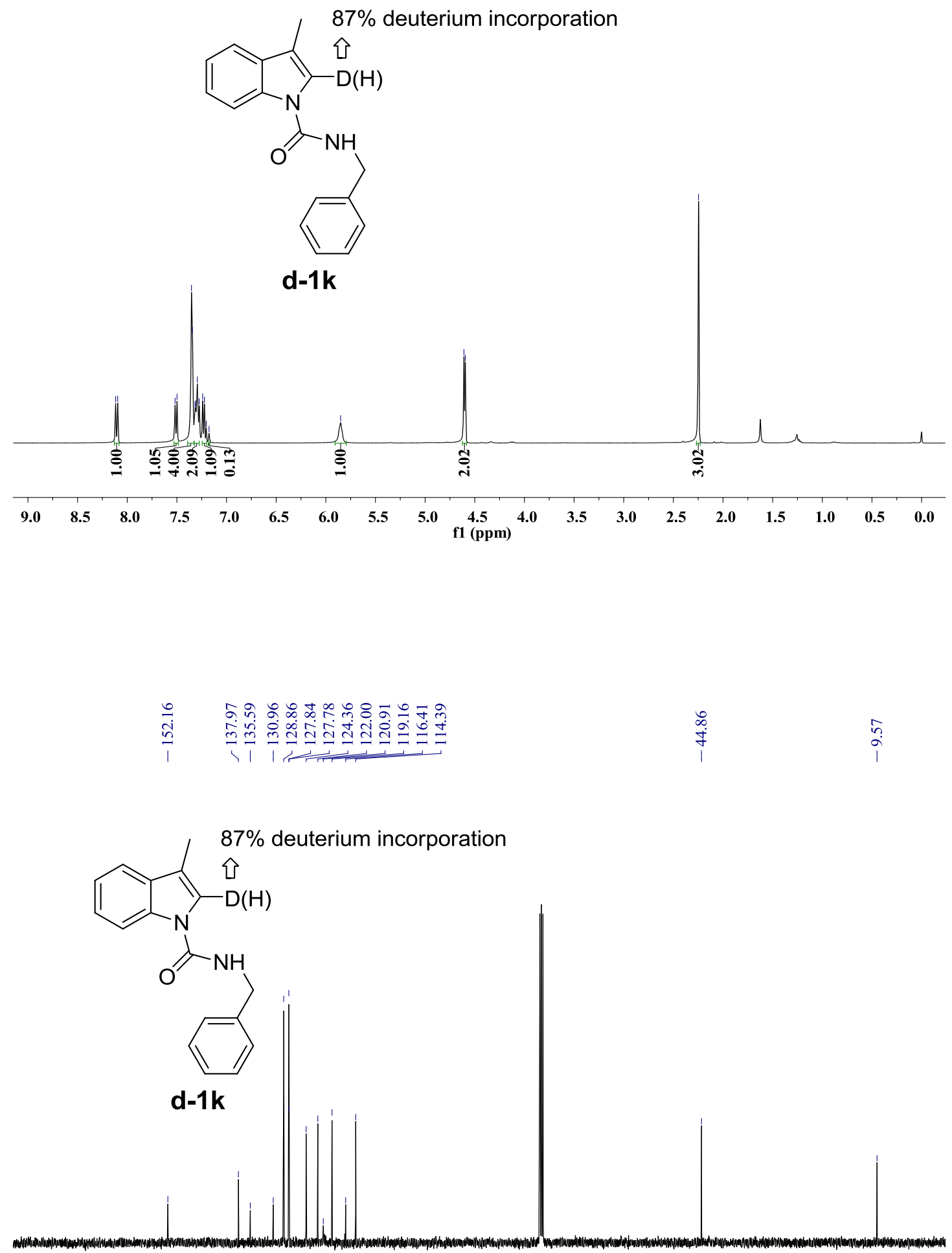

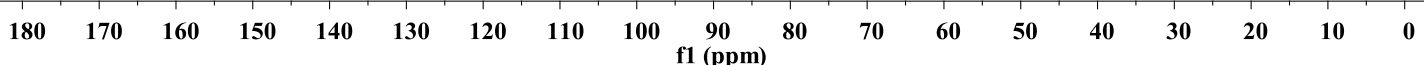


(32) The ${ }^{1} \mathrm{H}$ NMR and ${ }^{13} \mathrm{C}$ NMR spectrum for $\mathbf{1 t}$ (using $\mathrm{CDCl}_{3}$ as solvent)
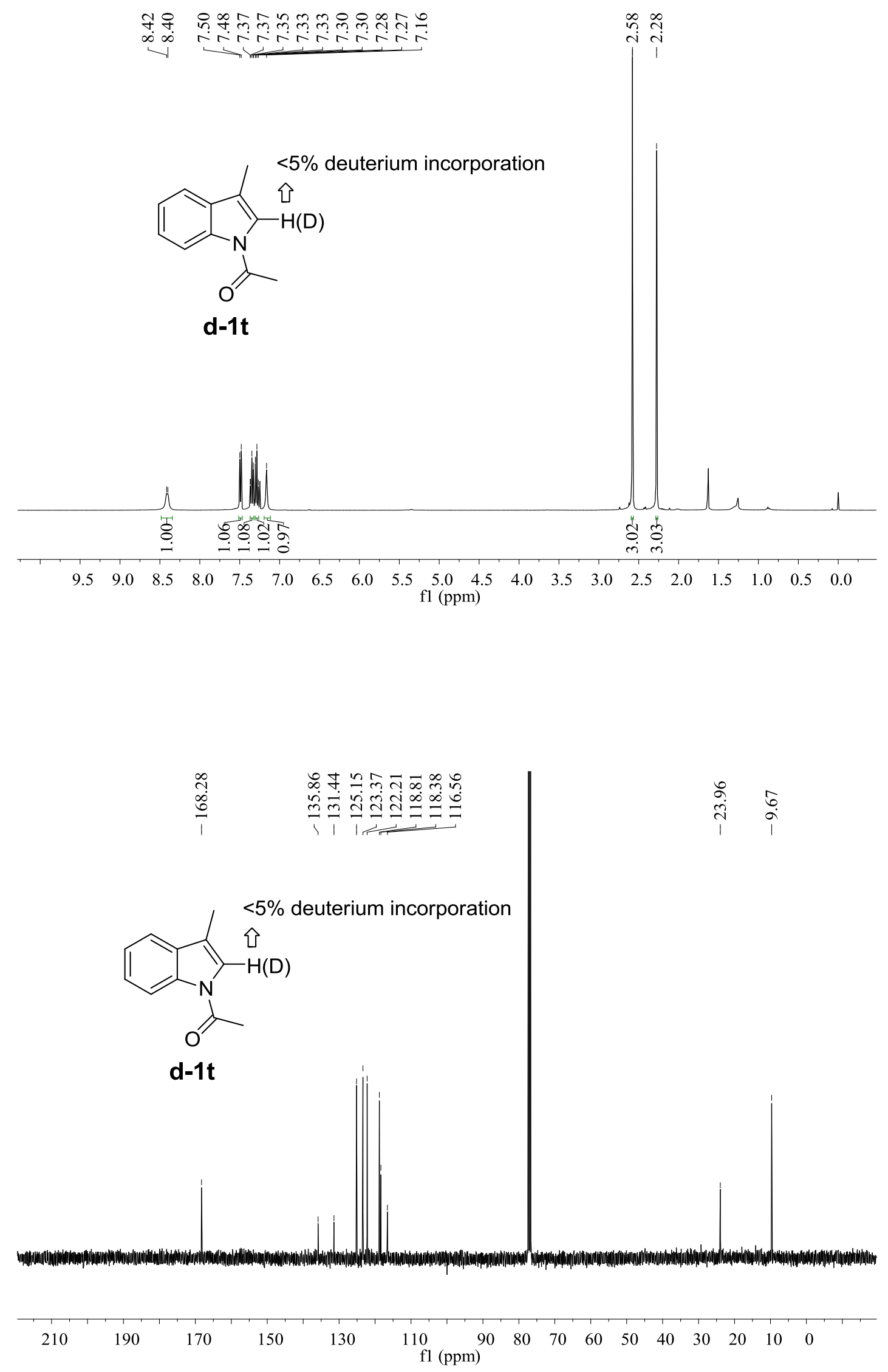
(33) The crude ${ }^{1} \mathrm{H}$ NMR spectrum for calculating KIE value via parallel reactions.
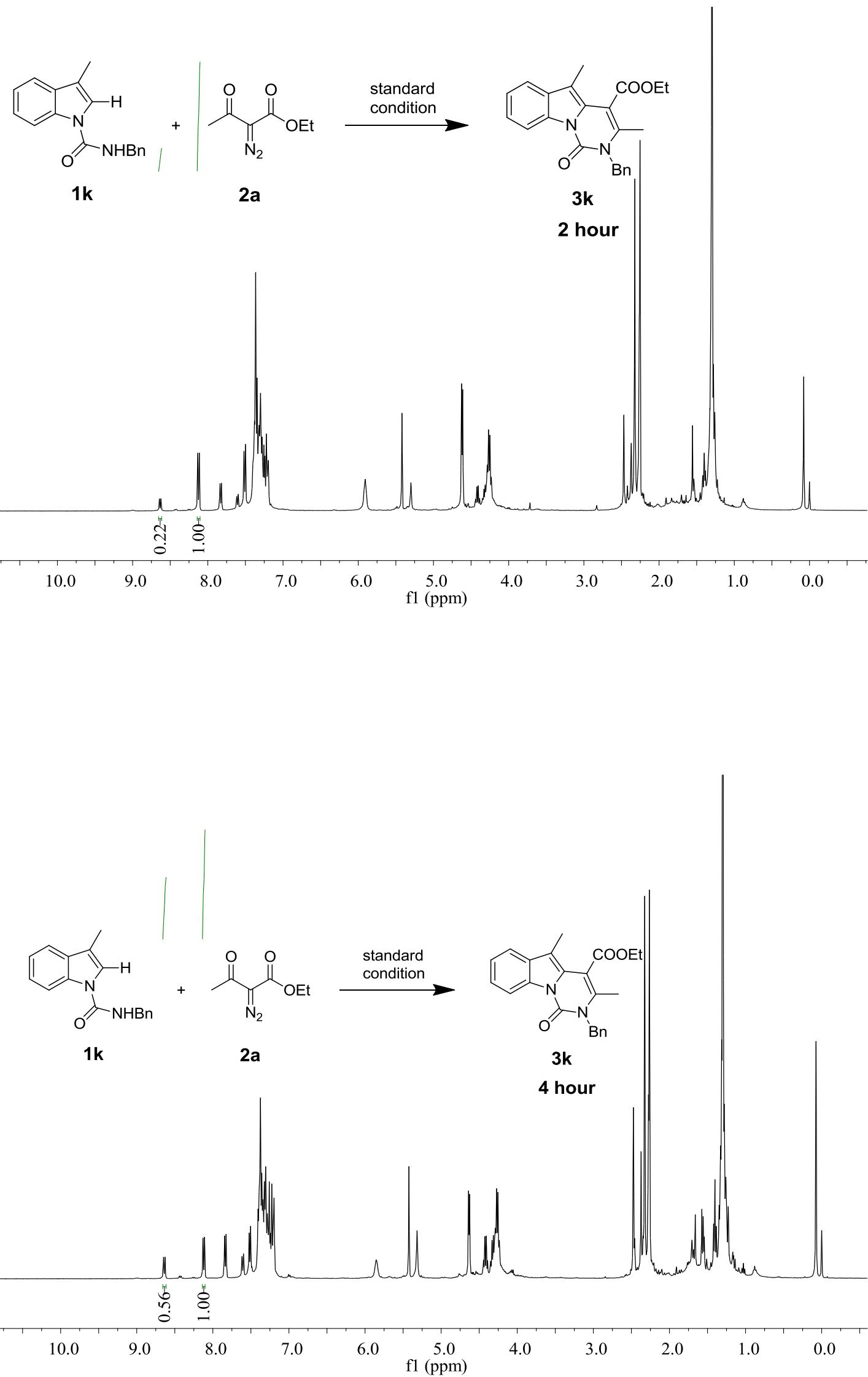

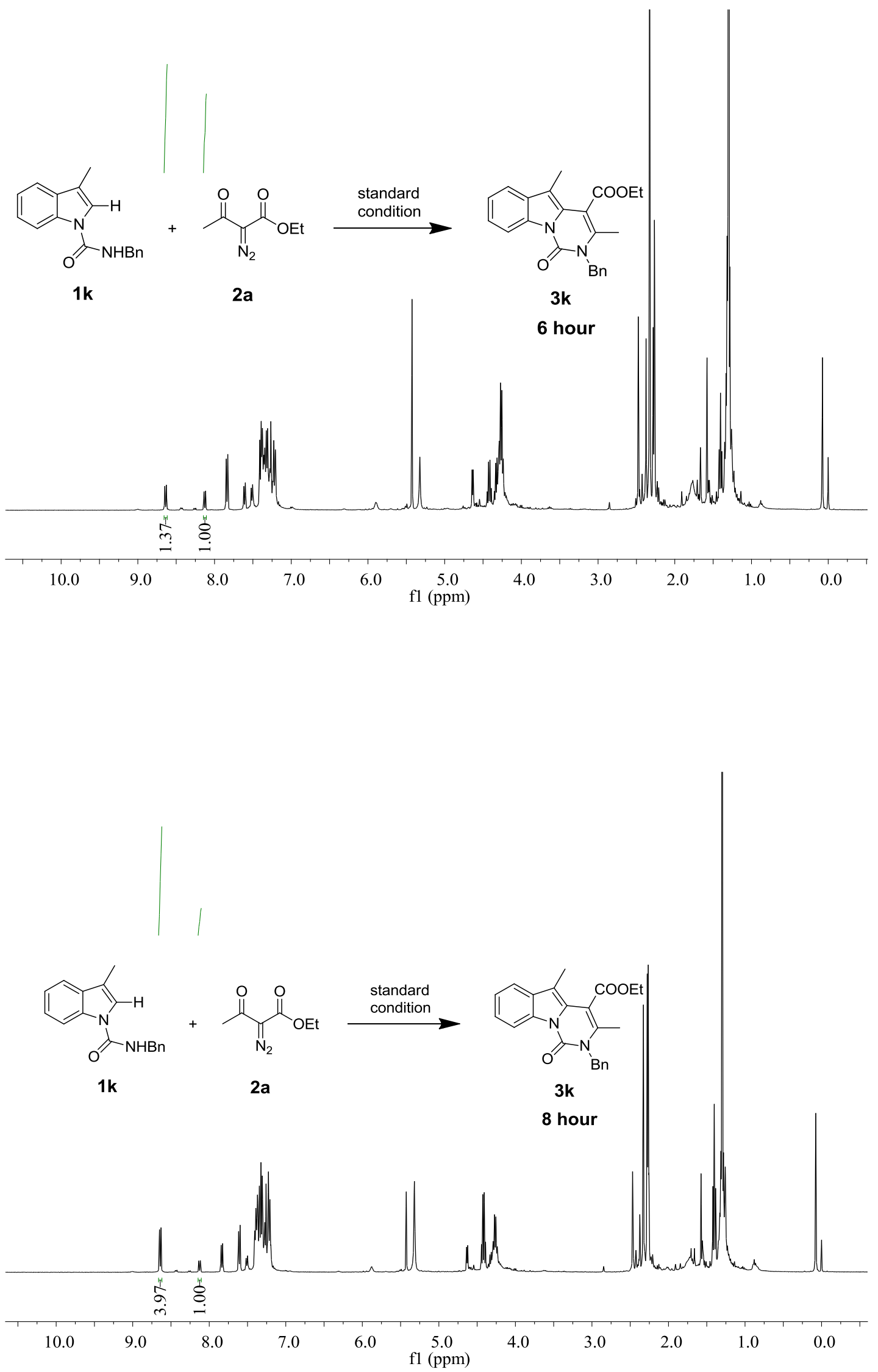

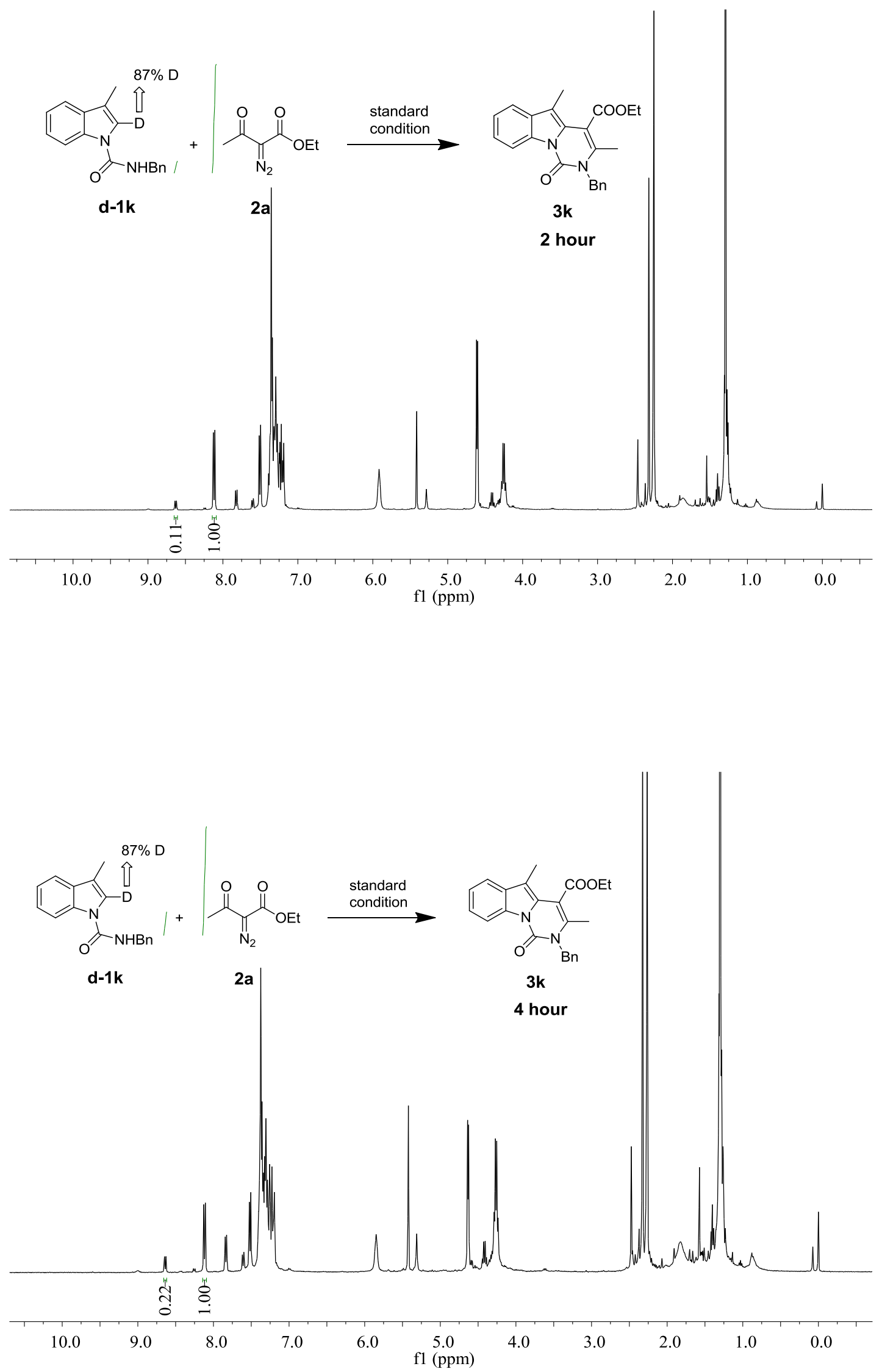

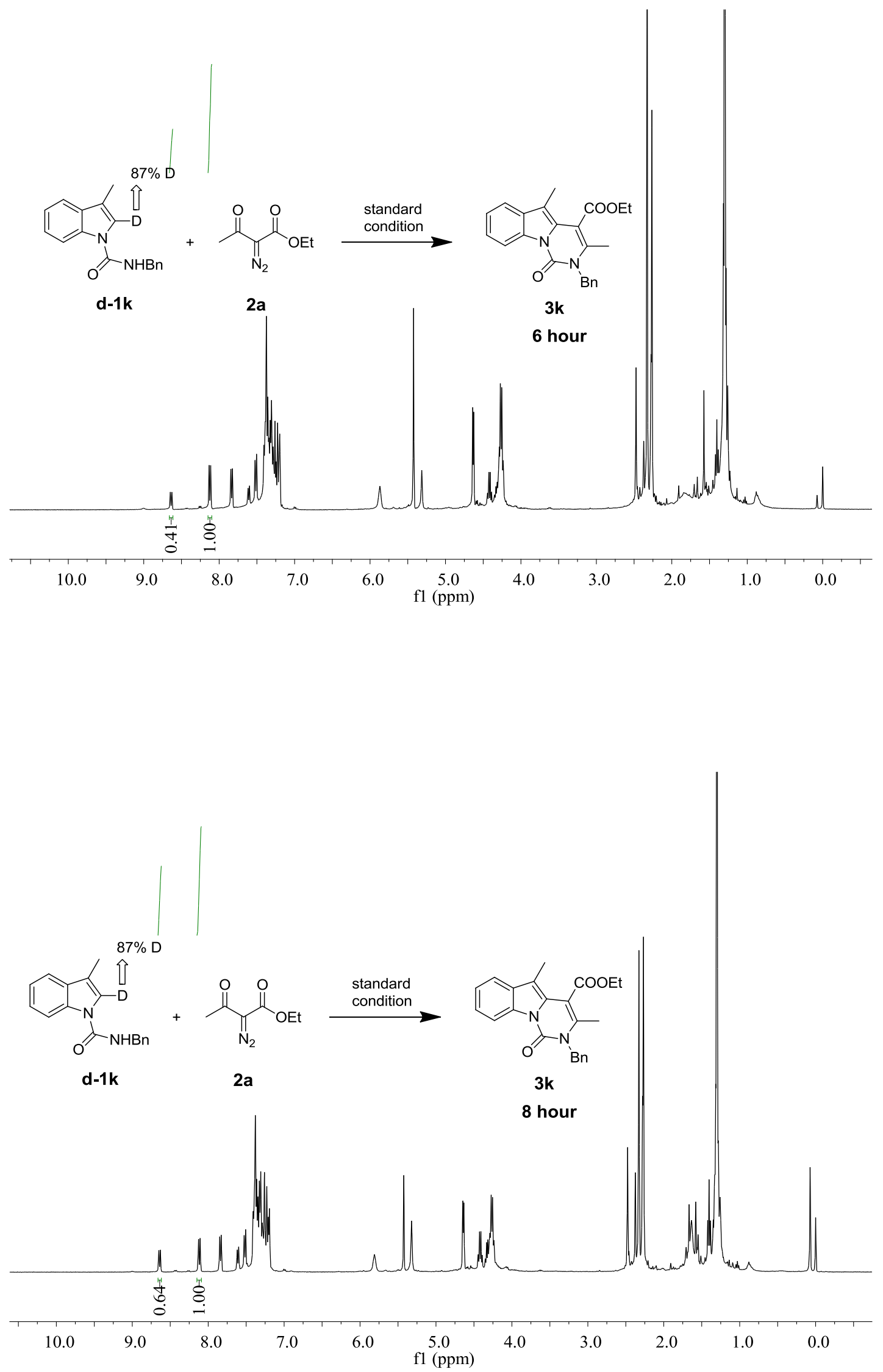